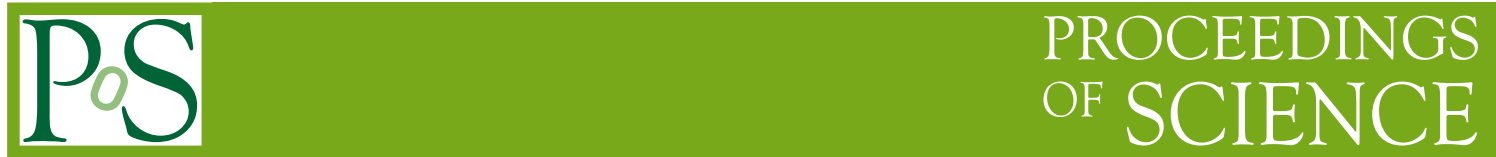

\title{
Indirect and direct dark matter detection
}

\section{Pierre Salati*}

LAPTH, Université de Savoie, CNRS

BP 110, 74941 Annecy-le-Vieux cedex, France

E-mail: salati@lapp.in2p3.fr

One of the favorite candidates that may solve the long standing astronomical dark matter puzzle is a massive particle with weak interactions. Such a species is predicted in the framework of supersymmetry or of extra dimension theories. Both extensions of the standard electro-weak model will soon be tested at the large hadron collider and neutralinos or Kaluza-Klein photons will be copiously produced should they exist. However, the observation in vitro of these putative particles will not prove that they pervade the ether. These lectures are devoted to the various methods - both direct and indirect - that aim at observing them in vivo. The dark matter species could collide on the nuclei of a terrestrial detector and leave an observable imprint. They are also expected to annihilate in pairs within the galactic halo and to produce visible radiations such as high-energy photons or neutrinos as well as rare antimatter cosmic rays. The hunt for the astronomical neutralinos or Kaluza-Klein particles to which I invite you here will take us deep underground, in the desert or in orbit around the Earth.

Cargèse Summer School: Cosmology and Particle Physics Beyond the Standard Models

July 30 - August 11, 2007

Institut d'Etudes Scientifiques de Cargese

\footnotetext{
* Speaker.
} 


\section{Contents}

1. Direct detection 3

1.1 A not too naïve toy model 4

1.2 Scattering cross section and nucleon content 11

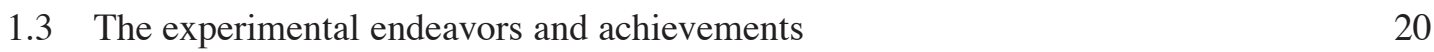

2. The messengers of DM annihilation 32

2.1 Cosmic ray transport : a short overview 32

2.2 TeV antiprotons : a new window 35

2.3 The positron signal 46

2.4 High-energy photons and the galactic center 62

3. DM substructures and the galactic lottery 69

$\begin{array}{lll}3.1 & \text { A statistical approach of galactic variance } & 71\end{array}$

3.2 Dark matter mini-spikes around black holes 83

3.3 The boost factor of $\Lambda-\mathrm{CDM}$ clumps as a conclusion $\quad 90$ 


\section{Direct detection}

Weakly interacting massive particles (hereafter WIMPs) may provide a natural explanation to the astronomical dark matter whose nature is still unresolved. Should these species exist, they would drift through the Milky Way. Because their interactions are so weak, they should pass through the Earth without much harm. Just a few of the impinging WIMPs are expected to collide elastically upon terrestrial nuclei, partially transferring to them their kinetic energy. Direct detection consists in observing the recoiled nuclei.

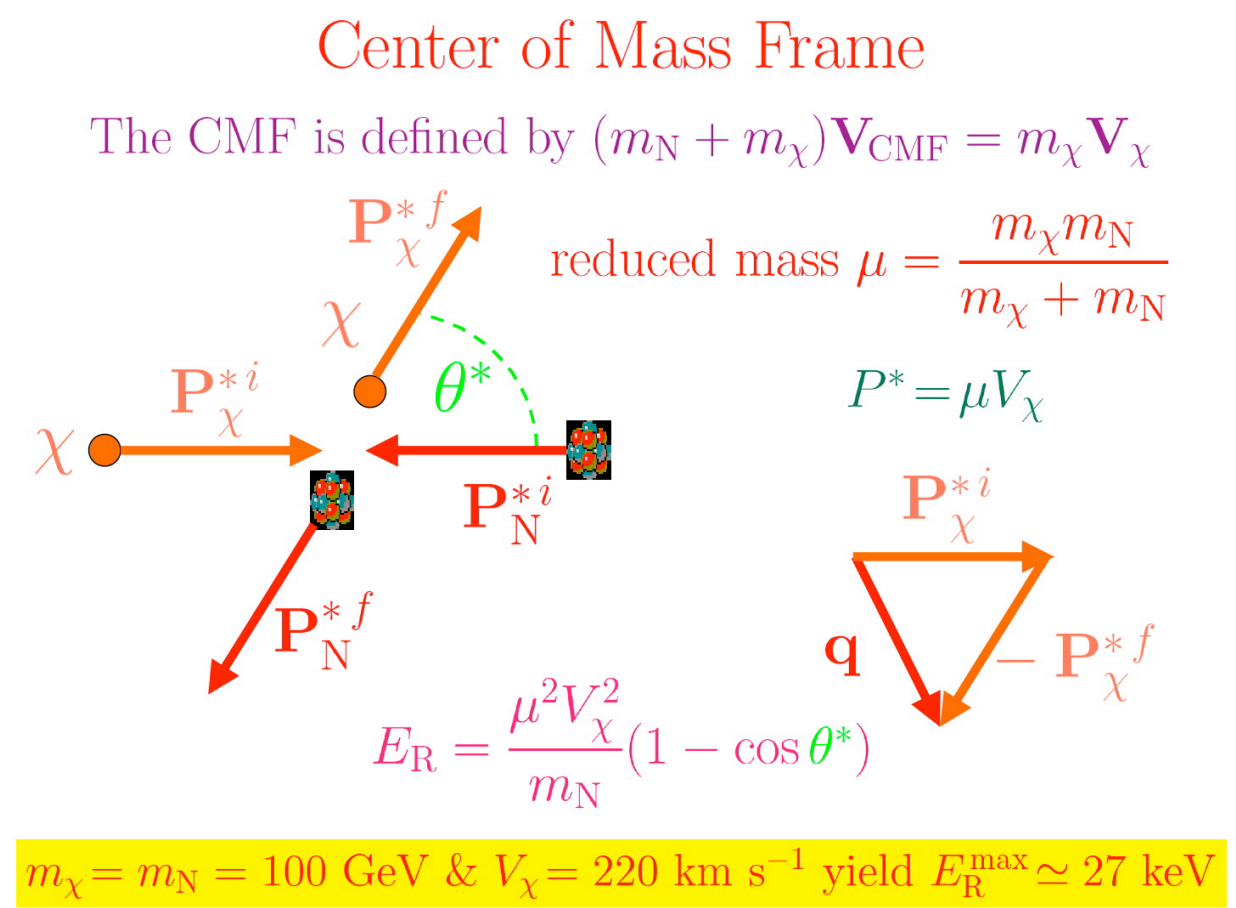

Figure 1: Kinematics of a WIMP-nucleus elastic collision as seen in the center of mass frame. In the laboratory, the WIMP impinges with velocity $\mathbf{V}_{\chi}$ on a nucleus at rest to which the energy $E_{\mathrm{R}}$ is transferred. The masses of the WIMP and of the nucleus are respectively denoted by $m_{\chi}$ and $m_{\mathrm{N}}$.

In the laboratory, an incoming WIMP with velocity $\mathbf{V}_{\chi}$ interacts upon a nucleus at rest to which a momentum $\mathbf{q}$ is transferred. The energy deposited in the detector by this single collision is

$$
E_{\mathrm{R}}=\frac{\mathbf{q}^{2}}{2 m_{\mathrm{N}}},
$$

where $m_{\mathrm{N}}$ is the nucleus mass. The recoil energy $E_{\mathrm{R}}$ is the signal which direct detection is looking for and may be expressed - see figure 1 - as a function of the recoil angle $\theta^{*}$ in the center of mass frame

$$
E_{\mathrm{R}}=\frac{\mu^{2} V_{\chi}^{2}}{m_{\mathrm{N}}}\left(1-\cos \theta^{*}\right),
$$

where $\mu$ denotes the reduced mass of the WIMP-nucleus system. 
Exercise $\mathbf{n}^{0}$ 1-a - Level [1] : Establish relation (1.2) and show that if a $100 \mathrm{GeV}$ WIMP with incoming velocity $V_{\chi}=220 \mathrm{~km} \mathrm{~s}^{-1}$ collides upon a $100 \mathrm{GeV}$ nucleus, the transferred energy may reach a maximal value of $27 \mathrm{keV}$.

\subsection{A not too naïve toy model}

The recoil energy $E_{\mathrm{R}}$ is quite small. Another difficulty of direct detection is the scarcity of the collisions between WIMPs and the nuclei of a terrestrial detector. In order to estimate the number of events per unit time, unit of recoil energy and unit of detector mass, we naïvely model the WIMP-nucleus interaction with the Lagrangian density

$$
\mathscr{L}_{\text {scalar }}=A g_{S}(\bar{\chi} \chi)\left(\bar{\Psi}_{\mathrm{N}} \Psi_{\mathrm{N}}\right)
$$

where $g_{S}$ denotes a scalar coupling. The WIMP is assumed here to scatter coherently on the $A$ nucleons of the target nucleus $\mathrm{N}$. This behaviour is generic of spin independent interactions for which the atomic number $A$ comes into play and not the nucleus spin. The scalar coupling is typically of order $g_{S} \sim \alpha_{\mathrm{em}} / M^{2}$ where $\alpha_{\mathrm{em}}=1 / 137$ is the fine-structure constant and $M$ is some scale at which physics beyond the standard model comes into play. As an example, that scalar coupling is $g_{S} \sim 7.3 \times 10^{-9} \mathrm{GeV}^{-2}$ for a scale $M=1 \mathrm{TeV}$.

Exercise $\mathbf{n}^{0} \mathbf{1}-\mathbf{b}-$ Level [2] : The cross section for the scattering process

$$
\chi\left(P_{1}\right)+\operatorname{nucleus}\left(k_{1}\right) \longrightarrow \chi\left(P_{2}\right)+\operatorname{nucleus}\left(k_{2}\right),
$$

is generically given by the well-known relation

$$
d \sigma\left|\mathbf{V}_{\chi}-\mathbf{V}_{\mathrm{N}}\right|=\frac{m_{\chi}}{P_{1}^{0}} \frac{m_{\mathrm{N}}}{k_{1}^{0}} \int d \tilde{P}_{2} d \tilde{k}_{2}(2 \pi)^{4} \delta^{4}\left(P_{1}+k_{1}-P_{2}-k_{2}\right) \mathscr{A},
$$

where $\mathscr{A}$ denotes the average over the initial spin states and the sum over the final spin states of the square of the amplitude. Show that the latter may be expressed as

$$
\mathscr{M}_{\text {scalar }}=\sqrt{\kappa} \times A g_{S} \times \bar{u}\left(P_{2}\right) u\left(P_{1}\right) \times \bar{u}\left(k_{2}\right) u\left(k_{1}\right) .
$$

The $\kappa$ coefficient is equal to 1 for Dirac fermions and to 4 for Majorana species. In the NR limit where the velocities of the particles are negligible with respect to their energies, establish that $\mathscr{A}$ is given by

$$
\mathscr{A} \equiv \frac{1}{4} \sum_{\text {spins }}\left|\mathscr{M}_{\text {scalar }}\right|^{2}=\kappa A^{2} g_{S}^{2}
$$

Compute the integral (1.5) in order to derive the differential cross section in the CMF

$$
\frac{d \sigma}{d \Omega^{*}}=\frac{\kappa}{4 \pi^{2}} A^{2} g_{S}^{2} \mu^{2}
$$

A few observations can be readily derived from the previous result. 
(i) Because the scattering is isotropic in the center of mass frame, the differential cross section is flat as a function of the recoil energy $E_{\mathrm{R}}$ and $d \sigma / d E_{\mathrm{R}} \equiv \sigma / E_{\mathrm{R}}^{\max }$. The recoil energy spectrum extends from $E_{\mathrm{R}}=0$ up to a maximal value of $E_{\mathrm{R}}^{\max }=2 \mu^{2} V_{\chi}^{2} / m_{\mathrm{N}}$. In our simplistic model, the total cross section may be expressed as

$$
\sigma=\frac{\kappa}{\pi} A^{2} g_{S}^{2} \mu^{2}
$$

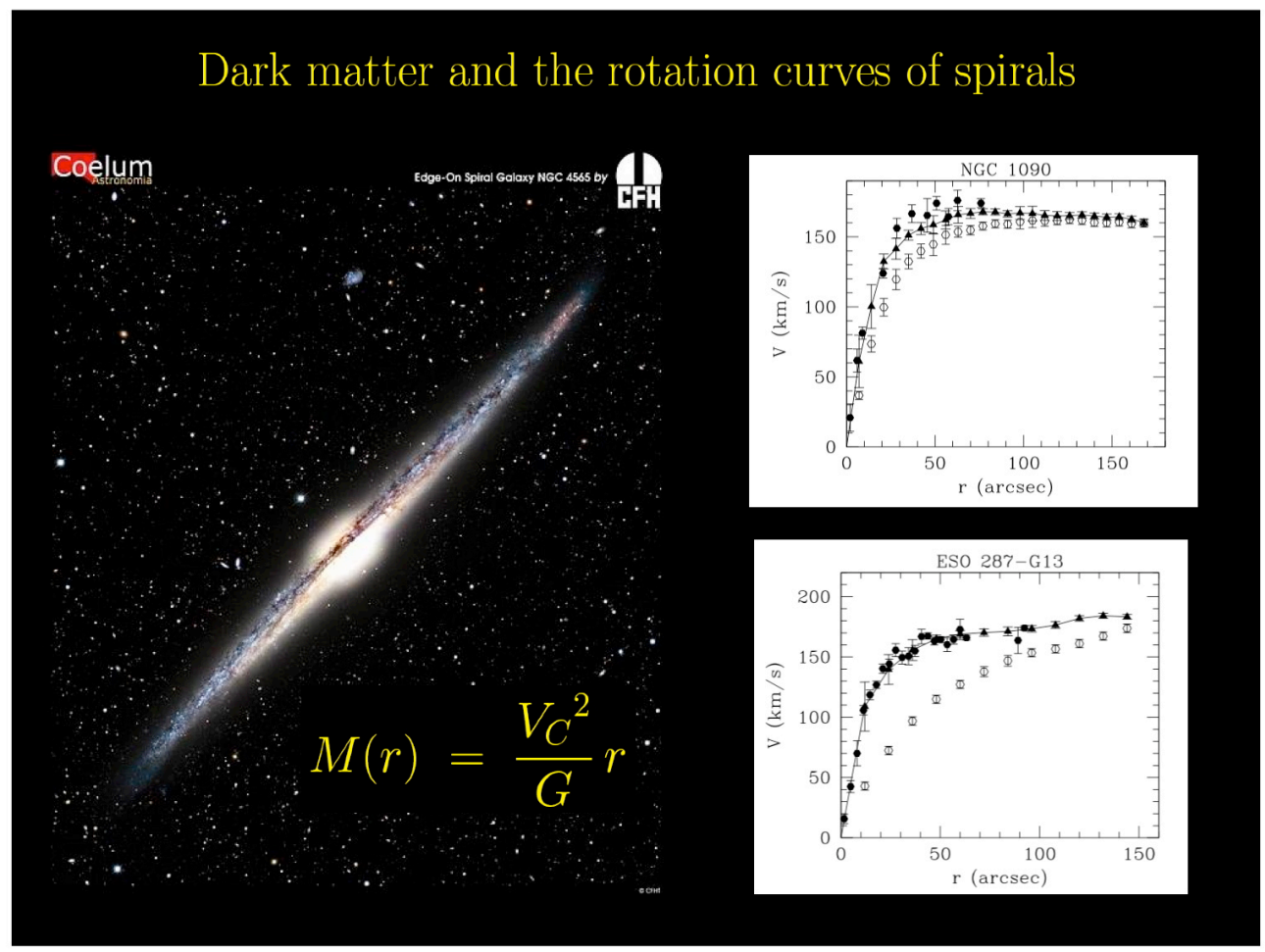

Figure 2: A large number of spiral galaxies have flat rotation curves that extend well beyond their optical radii. This illustration features on the left an optical image of the edge-on spiral NGC 4565 taken by the Canada France Hawaii Telescope and on the right the rotation curves of the NGC 1090 and ESO 287-G13 systems [1].

(ii) In order to compare among the various experiments whose detectors are made of different materials - for which the atomic number may vary appreciably - it is convenient to define the spin independent cross section on a single nucleon as the limit

$$
\sigma_{\mathrm{p}}^{\mathrm{SI}}=\lim _{m_{\chi} \rightarrow \infty} \sigma\left\{m_{\mathrm{N}}=m_{\mathrm{p}}, m_{\chi}\right\}=\frac{\kappa}{\pi} g_{S}^{2} m_{\mathrm{p}}^{2} .
$$

Setting as before the typical scale $M$ at $1 \mathrm{TeV}$ leads to a cross section of $\sigma_{\mathrm{p}}^{\mathrm{SI}} \sim 23.3$ zeptobarns ${ }^{1}$ on a single nucleon.

\footnotetext{
${ }^{1}$ One zeptobarn $(\mathrm{zb})=10^{-9}$ picobarn $=10^{-45} \mathrm{~cm}^{2}$.
} 
(iii) For spin independent interactions, the total scattering cross section varies with the atomic number $A$ of the target nucleus as

$$
\frac{\sigma}{\sigma_{\mathrm{p}}^{\mathrm{SI}}}=A^{4}\left\{1+\frac{m_{\mathrm{N}}}{m_{\chi}}\right\}^{-2},
$$

and scales as $A^{4}$ in the regime where $m_{\chi} \gg m_{\mathrm{N}}$. Heavy nuclei are therefore preferred since they are associated to much larger interaction cross sections and collision rates.

Each nucleus of a terrestrial detector is embedded inside a stream of WIMPs whose velocity distribution is $\mathrm{f}(\mathbf{v})$ in the laboratory frame. It may undergo a collision whose probability per unit time and unit of recoil energy is given by

$$
\frac{\mathrm{d} \Gamma}{\mathrm{d} E_{\mathrm{R}}}=\frac{\rho_{\odot}}{m_{\chi}} \int_{v_{\min }\left(E_{\mathrm{R}}\right)}^{v_{\max }} \mathrm{d}^{3} \mathbf{v} \mathrm{f}(\mathbf{v}) v \frac{d \sigma}{d E_{\mathrm{R}}}
$$

The DM density $\rho_{\odot}$ in the solar neighborhood is estimated to lie in the range between 0.2 and 0.8 $\mathrm{GeV} \mathrm{cm}{ }^{-3}$ and will be set equal to $0.3 \mathrm{GeV} \mathrm{cm}^{-3}$ hereafter. As the recoil spectrum is flat, the collision rate per unit mass of the detector is inferred to be

$$
\frac{\mathrm{d} R}{\mathrm{~d} E_{\mathrm{R}}}=\frac{\rho_{\odot}}{m_{\chi}} \frac{\sigma}{\sqrt{\pi} \mu^{2} V_{C}} \mathscr{T}\left(E_{R}\right)
$$

where the integral $\mathscr{T}\left(E_{R}\right)$ depends on the velocity distribution of the DM particles through

$$
\mathscr{T}\left(E_{R}\right)=\frac{\sqrt{\pi}}{2} V_{C} \int_{v_{\min }\left(E_{\mathrm{R}}\right)}^{v_{\max }} \frac{\mathrm{d}^{3} \mathbf{v}}{v} \mathrm{f}(\mathbf{v})
$$

This integral is normalized by the rotation velocity $V_{C} \simeq 220 \mathrm{~km} \mathrm{~s}^{-1}$ of the Milky Way - see figure 2. The upper bound $v_{\max }$ corresponds to the escape velocity from the Galaxy whereas the lower bound $v_{\min }$ is the critical value below which a WIMP cannot transfer the recoil energy $E_{R}$ whatever the scattering angle $\theta^{*}$ in the center of mass frame

$$
\frac{2 \mu^{2} v_{\min }^{2}}{m_{\mathrm{N}}}=E_{R} .
$$

A key ingredient in the calculation of the recoil spectrum $\mathrm{d} R / \mathrm{d} E_{\mathrm{R}}$ is the WIMP velocity distribution $\mathrm{f}(\mathbf{v})$ with respect to the Earth. 
Exercise $\mathbf{n}^{0} \mathbf{1 - c}-$ Level [2] : The model of the isothermal sphere.

In the absence of collisions, WIMPs undergoing the action of the gravitational potential $\Phi$ behave in phase-space like an incompressible fluid whose density $\mathrm{f}(\mathbf{r}, \mathbf{v}, t)$ follows the Vlasov equation

$$
\frac{\partial \mathrm{f}}{\partial t}+(\mathbf{v} \cdot \nabla) \mathrm{f}-\left(\nabla \Phi \cdot \nabla_{v}\right) \mathrm{f}=0
$$

Show that any function $\mathrm{f}$ of the mechanical energy per unit mass $E=v^{2} / 2+\Phi(\mathbf{r})$ is a stationary solution of (1.16). Let us choose the Maxwell-Boltzmann distribution $f=C \exp \left(-E / v^{2}\right)$, where $v$ denotes the typical velocity dispersion of the particles. By integrating out the velocities, establish that the mass density is given by

$$
\rho(\mathbf{r})=\rho_{c} \exp \left\{-\Phi(\mathbf{r}) / v^{2}\right\} .
$$

Assuming spherical symmetry, solve the Poisson equation

$$
\Delta \Phi=\frac{1}{r^{2}} \frac{d}{d r}\left\{r^{2} \frac{d \Phi}{d r}\right\}=4 \pi G \rho(r) .
$$

for the scale invariant solution $\rho=A r^{\alpha}$. Compute $A$ and $\alpha$ in order to derive the specific form for the mass density of an isothermal sphere

$$
\rho(r)=\frac{v^{2}}{2 \pi G} \frac{1}{r^{2}} .
$$

Show that if the dark matter inside a spiral galaxy follows that profile and if it dominates the dynamics of the system, the rotation curve is flat with velocity $V_{C}=\sqrt{2} v$.

In the very popular model of the isothermal cored sphere [2], the WIMP velocity distribution with respect to the galactic frame is the Maxwell-Boltzmann function

$$
f(\mathbf{v})=\left(\pi V_{C}^{2}\right)^{-3 / 2} \exp \left(-v^{2} / V_{C}^{2}\right) .
$$

If we neglect the Earth motion with respect to the Milky Way DM halo and set the upper bound $v_{\max }$ at infinity - which numerically is a fair approximation - we get a very simple form for the velocity integral

$$
\mathscr{T}\left(E_{R}\right)=\exp \left(-v_{\min }^{2} / V_{C}^{2}\right)=\exp \left(-E_{R} / E_{R}^{0}\right) .
$$

The recoil spectrum decreases exponentially with a variation scale set by

$$
E_{R}^{0}=2 \mu^{2} V_{C}^{2} / m_{\mathrm{N}},
$$

For a ${ }^{73} \mathrm{Ge}$ target nucleus and a $60 \mathrm{GeV}$ impinging WIMP, we find a typical recoil energy of $E_{R}^{0}=16$ $\mathrm{keV}$. The Earth actually rotates around the galactic center and moves with respect to the Milky Way DM halo with a velocity comparable to $V_{C}$

$$
v_{e}=V_{C}\left\{1.05+0.07 \cos \left\{\frac{2 \pi\left(t-t_{P}\right)}{1 \mathrm{yr}}\right\}\right\} .
$$

The orbital motion around the Sun adds up to the galactic rotation so that $v_{e}$ is maximal on June the 2nd \pm 1.3 days and minimal 6 months later. This results into an annual modulation of the expected signal which the experiment DAMA has reported to have observed [3]. 
Exercise $\mathbf{n}^{0}$ 1-d - Level [2] : As a consequence of the Earth motion, the correct WIMP velocity distribution in the laboratory is given by the same function $\mathrm{f}$ as before, where the argument $\mathbf{v}$ is replaced now by the velocity $\mathbf{v}_{\mathrm{G}}=\mathbf{v}+\mathbf{v}_{e}$ as seen in the galactic frame. Show that the simple expression (1.21) for the velocity integral becomes

$$
\mathscr{T}\left(E_{R}\right)=\frac{\sqrt{\pi}}{4} \frac{V_{C}}{v_{e}}\left\{\operatorname{Erf}\left(\frac{v_{\min }+v_{e}}{V_{C}}\right)-\operatorname{Erf}\left(\frac{v_{\min }-v_{e}}{V_{C}}\right)\right\}
$$

where Erf denotes the error function.

The two panels of figure 3 feature the recoil spectra of ${ }^{73}$ Ge target nuclei impacted by WIMPs of various masses. When the motion of the Earth with respect to the Milky Way DM halo is taken into account - right panel - the spectra flatten. An annual variation of the slope of the recoil spectrum is expected, albeit with a much smaller amplitude as presented here.
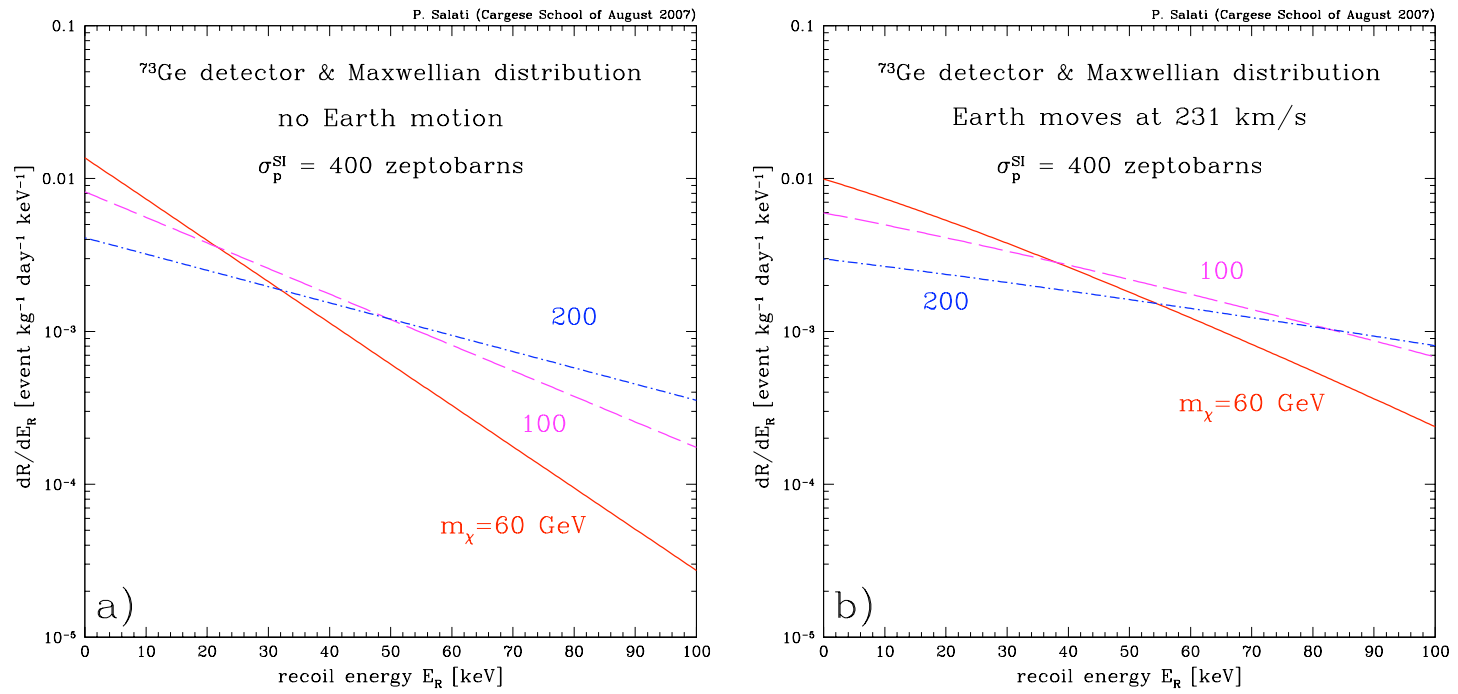

Figure 3: The recoil energy spectrum on ${ }^{73} \mathrm{Ge}$ target nuclei is featured for three values of the WIMP mass. In the left panel, the Earth is taken at rest with respect to the Milky Way DM halo whereas in the right panel, it moves at a velocity of $231 \mathrm{~km} \mathrm{~s}^{-1}$. Recoil spectra are flatter when the Earth motion is taken into account.

If a detector of mass $\mathscr{M}$ is embedded inside the WIMP stream during a period $\mathscr{T}$, its exposure will amount to $\mathscr{E} \equiv \mathscr{M} \times \mathscr{T}$ and is expressed in units of $\mathrm{kg} \times$ day. Recoil events are detected above the threshold $E_{\text {th }}$ up to an energy $E_{\max }$. During the period of observation - characterized by the exposure $\mathscr{E}$ - the expected number $n_{\text {theo }}$ of detectable collisions is given by the integral of the differential rate (1.13) over the appropriate recoil energies

$$
n_{\text {theo }}=\mathscr{E} \times \int_{E_{\text {th }}}^{E_{\max }} \frac{\mathrm{d} R}{\mathrm{~d} E_{\mathrm{R}}} \mathrm{d} E_{\mathrm{R}}
$$

In the naïve approach where the galactic rotation of the Earth is ignored and the upper bound $E_{\max }$ 
is set at infinity, this number of events simplifies into

$$
n_{\text {theo }} \simeq 4.14 \times 10^{-7} \text { events kg }{ }^{-1} \text { day }^{-1}\left\{\frac{\sigma_{\mathrm{p}}^{\mathrm{SI}}}{1 \mathrm{zb}}\right\} A^{2}\left\{\frac{\sqrt{x}-1}{x}\right\} e^{-\alpha x},
$$

where $\alpha=E_{\mathrm{th}} / 2 m_{\mathrm{N}} V_{C}^{2}$ and the mass parameter $x$ is defined as $x=\left(1+m_{\mathrm{N}} / m_{\chi}\right)^{2}$. The sensitivity

\section{We require that $n_{\text {theo }} \leq 2.3$ (90\% CL for a null observation)}

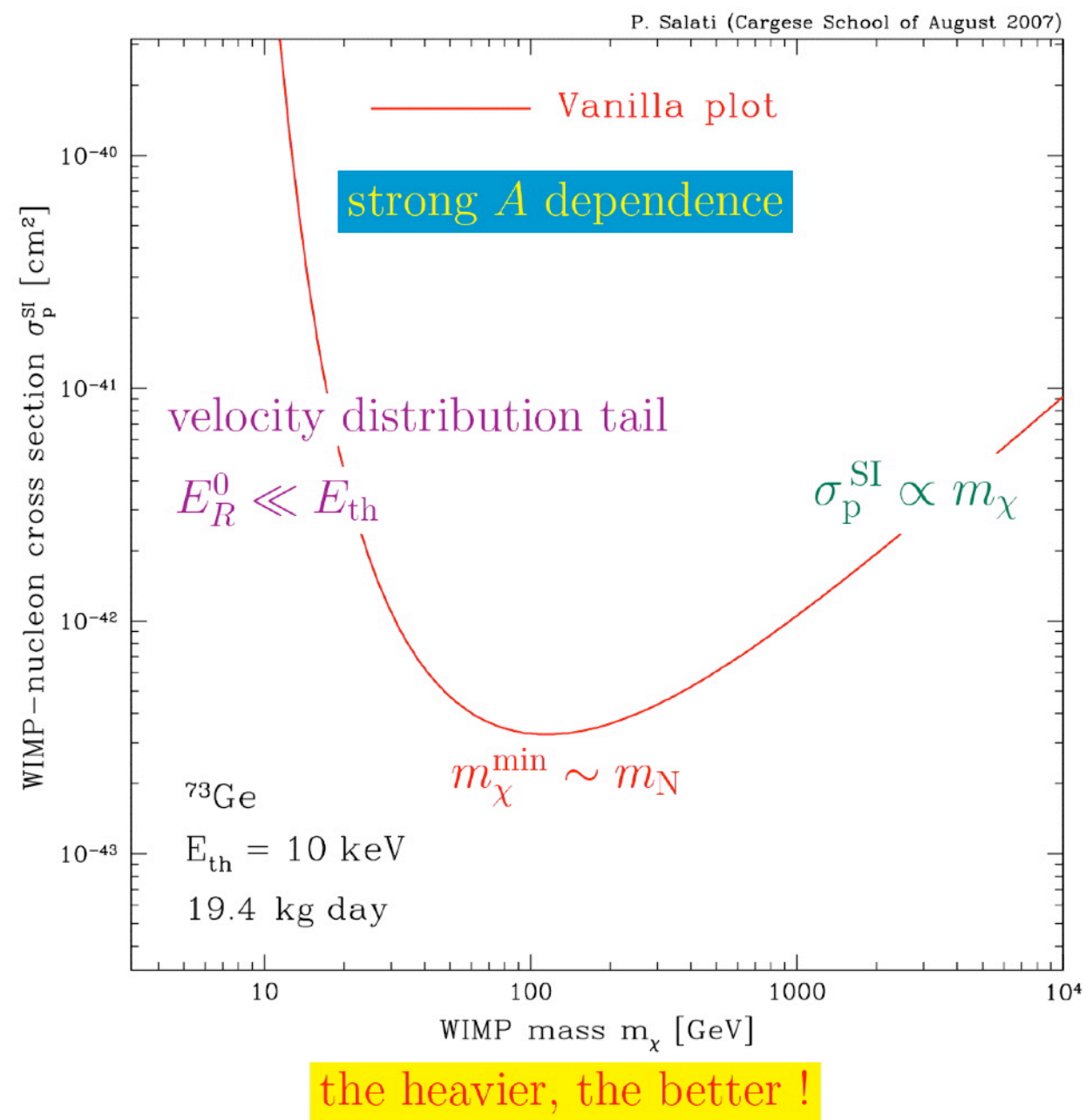

Figure 4: The region in the $\left(\sigma_{\mathrm{p}}^{\mathrm{SI}}, m_{\chi}\right)$ plane extending above the red solid line can be potentially reached and excluded in the case of a null observation - by an experiment like CDMS [4]. A ${ }^{73} \mathrm{Ge}$ detector has been chosen here with a recoil energy threshold of $10 \mathrm{keV}$ and an exposure of $19.4 \mathrm{~kg}$ day as in their first run at the Soudan underground facility.

of each experiment can be gauged with the help of relation (1.25). Cross sections for which at least a few events are expected - therefore for which $n_{\text {theo }}$ exceeds a number of order unity - can be probed. Conversely in the case of a null observation, Poisson statistics implies that $n_{\text {theo }}$ cannot exceed a value of 2.3 at the $90 \%$ confidence level. This translates into the exclusion plot of figure 4 where the area above the red solid line can be probed and rejected if no event is observed. Three 


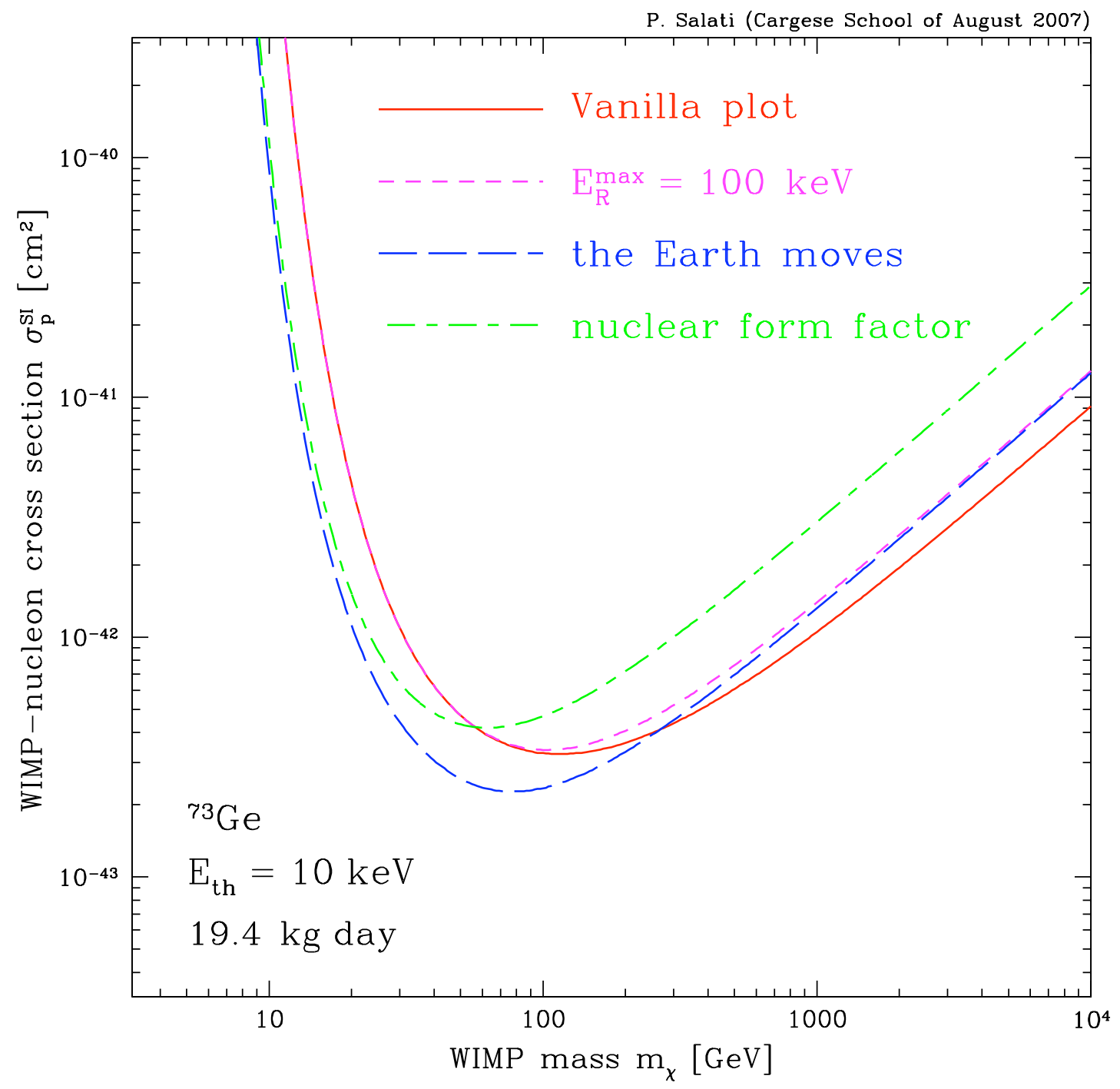

Figure 5: Same exclusion plot as before. The naïve vanilla red solid line is successively refined to yield the green short and long-dashed curve. Because both limits are not very different, the vanilla exclusion plot could be safely used for pedagogical purposes.

regions can be delineated depending on the WIMP mass. For small values of $m_{\chi}$, the typical energy $E_{R}^{0}$ that sets the scale of the recoil spectrum is much smaller than the threshold energy $E_{\mathrm{th}}$ of the experiment. The only WIMPs which can leave a detectable imprint must belong to the high-energy tail of the velocity distribution hence the sharp edge of the excluded domain as $m_{\chi}$ decreases. In the opposite regime where $m_{\chi}$ is large, the limit set on the cross section $\sigma_{\mathrm{p}}^{\mathrm{SI}}$ varies linearly with the WIMP mass. The recoil energy is no longer a limitation but the DM particle density is. Actually the larger $m_{\chi}$, the smaller the number density $n_{\chi}=\rho_{\odot} / m_{\chi}$ and the smaller the expected number of events $n_{\text {theo }} \propto 1 / m_{\chi}$. Finally, in the regime where the threshold energy is small with respect to the typical energy $2 m_{\mathrm{N}} V_{C}^{2}$ - for small values of the $\alpha$ parameter in relation (1.26) - the expected number of events $n_{\text {theo }}$ is the largest and the limit on $\sigma_{\mathrm{p}}^{\mathrm{SI}}$ is the strongest when nuclei and WIMPs 
have equal masses. Notice also the strong dependence of the expected number of events $n_{\text {theo }}$ with the atomic number $A$ of the target material. It is not surprising therefore if the best limit obtained on the WIMP-nucleon spin independent cross section has been set [5] by using xenon for which $A=131$.

The red solid line of figure 4 translates relation (1.26) and is based on a few simplistic assumptions. This vanilla curve has been refined in figure 5. The magenta short-dashed limit is derived by setting now the upper bound $E_{\max }$ on detectable recoil energies at $100 \mathrm{keV}$ [4]. If in addition the Earth motion is taken into account, we get the blue long-dashed frontier. Finally, because a fraction only of the nucleus is probed by an impinging WIMP with a large enough transfer momentum $\mathbf{q}$, the spin independent cross section is reduced by a nuclear form factor which mostly comes into play for heavy WIMPs. This yields the green short and long-dashed line.

\subsection{Scattering cross section and nucleon content}

At the microscopic level - described by the fundamental theory chosen to extend the standard electro-weak model - supersymmetric neutralinos and Kaluza-Klein photons interact actually on the constituents of the nucleons, hence an effective WIMP-nucleus scattering cross section which needs to be carefully derived and which incorporates such effects as the contribution of quarks and gluons to the interaction on a single nucleon or the way the nucleons come into play inside a given target nucleus. If the fundamental Lagrangian of the theory is in general well-defined, the derivation of the effective WIMP-nucleus scattering cross section suffers from large uncertainties as discussed now.

To commence, let us parameterize the effective coupling of the WIMP $\chi$ by the vertex $\bar{\chi} \Gamma \chi$ where $\Gamma$ stands for some combination of Dirac matrices. In direct detection, the amplitude of the diffusion relies upon the matrix element $\langle 2|\bar{\chi} \Gamma \chi| 1\rangle$, where a DM particle with initial momentum $P_{1}$ scatters on a nucleus and gets the momentum $P_{2}$. In supersymmetry, the neutralino is a Majorana fermion. Both particles and antiparticles are one and the same species so that the corresponding quantum field operator may be Fourier expanded as

$$
\chi(x)=\int \tilde{d k} \sum_{\alpha=1,2}\left\{a(\vec{k}, \alpha) u(\vec{k}, \alpha) e^{-i k x}+a^{\dagger}(\vec{k}, \alpha) v(\vec{k}, \alpha) e^{i k x}\right\},
$$

where both the creation $a$ and annihilation $a^{\dagger}$ operators are present in $\chi$ and $\bar{\chi}$.

Exercise $\mathbf{n}^{0}$ 1-e - Level [2] : Show that

$$
\langle 2|\bar{\chi} \Gamma \chi| 1\rangle=\bar{u}(2) \Gamma u(1)-\bar{v}(1) \Gamma v(2) .
$$

The 4-spinors $u$ and $v$ are related though charge conjugation by $u=v^{\mathrm{c}} \equiv C \bar{v}^{\mathrm{T}}$ where $C$ is equal to $i \gamma^{2} \gamma^{0}$ up to a phase in the 4-spinor Dirac representation. Infer from above that

$$
\langle 2|\bar{\chi} \Gamma \chi| 1\rangle=\bar{u}(2)\left\{\Gamma-C \Gamma^{\mathrm{T}} C\right\} u(1) .
$$

Compute the previous expression for $\Gamma=1$ (scalar), $\gamma^{\mu}$ (vector), $\sigma^{\mu v}=(i / 2)\left[\gamma^{\mu}, \gamma^{v}\right]$ (tensor), $\gamma^{5} \gamma^{\mu}$ (axial) and $\gamma^{5}$ (pseudo-scalar). Show that the vector and tensor contributions vanish exactly. How does the pseudo-scalar contribution behave in the non-relativistic limit? 
In this section, we shall restrict our discussion to supersymmetric models. In the non-relativistic regime of direct detection, we are therefore left only with an axial and/or a scalar effective coupling of the neutralino to the nucleus.

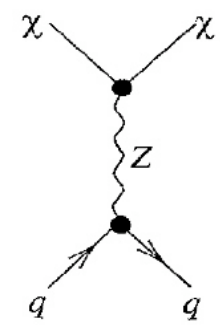

Fierz

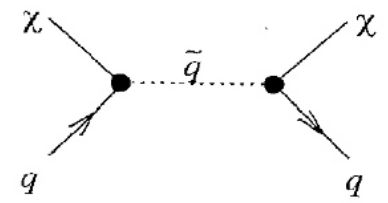

Figure 6: Feynman diagrams contributing to the axial elastic scattering of neutralinos off quarks and eventually to the spin dependent neutralino-nucleus scattering cross section.

Axial coupling - Once a particular supersymmetric model has been selected, the microscopic couplings $\mathrm{d}_{q}$ that come into play in the axial neutralino-quark interaction

$$
\mathscr{L}_{\mathrm{A}}=\mathrm{d}_{q}\left(\bar{\chi} \gamma^{\mu} \gamma_{5} \chi\right)\left(\bar{q} \gamma_{\mu} \gamma_{5} q\right)
$$

are determined once and for all. The matrix element of the quark axial-vector current inside a nucleon may be extracted from data on polarized deep-inelastic scattering

$$
\left\langle n\left|\bar{q} \gamma_{\mu} \gamma_{5} q\right| n\right\rangle=2 s_{\mu}^{(n)} \Delta q^{(n)}
$$

where $n$ stands for either the neutron or the proton. The parameters $\Delta q^{\mathrm{p}}$ describe the amount of spin carried out by the quark flavors $q$ inside the proton. They have been measured by several experiments which have reported different results as indicated in table 1.

\begin{tabular}{|c||cccc|}
\hline & NQM & EMC & SMC & HERMES \\
\hline \hline$\Delta u^{\mathrm{p}}$ & 0.93 & 0.746 & 0.80 & 0.842 \\
$\Delta d^{\mathrm{p}}$ & -0.33 & -0.508 & -0.46 & -0.427 \\
$\Delta s^{\mathrm{p}}$ & 0 & -0.226 & -0.12 & -0.085 \\
$\Delta \Sigma^{\mathrm{p}}$ & 0.60 & 0.012 & 0.22 & 0.33 \\
$\Gamma^{\mathrm{p}}$ & 0.188 & 0.114 & 0.136 & 0.140 \\
\hline
\end{tabular}

Table 1: Quark spin content of the proton determined from the $\mathrm{SU}(3)$ naïve quark model (NQM) [6] and from the first moment $\Gamma^{\mathrm{p}}$ of the spin dependent proton structure function $g_{1}^{\mathrm{p}}\left(x, Q^{2}\right)$ as measured by the EMC [7], SMC [8] and HERMES [9] collaborations. The quarks contribute an overall fraction of $\Delta \Sigma^{\mathrm{p}}$ to the spin of the proton.

The quark contributions to the spin of the neutron are obtained by an isospin rotation exchanging the up and down quarks so that $\Delta u^{\mathrm{n}} \equiv \Delta d^{\mathrm{p}}$ and $\Delta d^{\mathrm{n}} \equiv \Delta u^{\mathrm{p}}$ whereas $\Delta s^{\mathrm{n}} \equiv \Delta s^{\mathrm{p}}$. In the naïve parton model, the probability for a quark species $q$ with electric charge $e_{q}$ to carry a fraction $x$ of the momentum of the nucleon $n$ inside which it is embedded is described by the function $q^{n}(x)$. The spin dependent nucleon structure function $g_{1}^{(n)}(x)$ is defined as

$$
g_{1}^{(n)}(x)=\frac{1}{2} \sum_{q} e_{q}^{2}\left\{q^{(n)}(\uparrow, x)-q^{(n)}(\downarrow, x)\right),
$$


where the probability density $q^{(n)}(\uparrow, x)$ (or $q^{(n)}(\downarrow, x)$ ) refers to a quark with the same (or opposite) helicity as its host nucleon.

Exercise $\mathbf{n}^{0}$ 1-f - Level [1] : Integrating the spin dependent structure function $g_{1}^{(n)}(x)$ over $x$ leads to the first moment

$$
\Gamma^{(n)}=\int_{0}^{1} d x g_{1}^{(n)}(x)
$$

Show that the fraction $\Delta \Sigma^{(n)}$ of the nucleon spin that is carried out by the quarks may be expressed as

$$
\Delta \Sigma^{(n)}=\sum_{q} \Delta q^{(n)}=\sum_{q} \int_{0}^{1} d x\left\{q^{(n)}(\uparrow, x)-q^{(n)}(\downarrow, x)\right\}
$$

Relate the first moment $\Gamma^{(n)}$ and the quark spin fraction $\Delta \Sigma^{(n)}$ to the up-quark parameter

$$
\Delta u^{(n)}=6 \Gamma^{(n)}-\frac{1}{3} \Delta \Sigma^{(n)},
$$

and establish also that

$$
\Delta d^{(n)}+\Delta s^{(n)}=\frac{4}{3} \Delta \Sigma^{(n)}-6 \Gamma^{(n)} .
$$

Are those relations satisfied by the measurements presented in table 1 ? Any comment?

The effective coupling of the neutralino axial current to the nucleon spin may be parameterized by

$$
\mathscr{L}_{\mathrm{A}}=\left\{\sum_{\mathrm{u}, \mathrm{d}, \mathrm{s}} 2 \mathrm{~d}_{q} \Delta q^{(n)} \equiv 2 \sqrt{2} G_{F} a_{(n)}\right\}\left(\bar{n} s_{\mu} n\right)\left(\bar{\chi} \gamma^{\mu} \gamma_{5} \chi\right) .
$$

The next step is the calculation of the matrix element of the nucleon spin operators $\left(\bar{n}_{S_{\mu}} n\right)$ inside the nuclear state $|N\rangle$. At zero momentum transfer $\mathbf{q}$, this translates into the evaluation of the average proton spin

$$
\left\langle S_{\mathrm{p}}^{\mu}\right\rangle \equiv\left\langle\mathrm{N}\left|\left(\overline{\mathrm{p}} s^{\mu} \mathrm{p}\right)\right| \mathrm{N}\right\rangle
$$

and neutron spin

$$
\left\langle S_{\mathrm{n}}^{\mu}\right\rangle \equiv\left\langle\mathrm{N}\left|\left(\overline{\mathrm{n}} s^{\mu} \mathrm{n}\right)\right| \mathrm{N}\right\rangle
$$

inside the target nucleus $\mathrm{N}$. The expectation values $\left\langle S_{\mathrm{p}}\right\rangle$ and $\left\langle S_{\mathrm{n}}\right\rangle$ measure the amount of spin carried out respectively by the proton and the neutron groups inside the target nucleus. If we define now the parameter $\Lambda$ by

$$
a_{\mathrm{p}}\left\langle S_{\mathrm{p}}^{\mu}\right\rangle+a_{\mathrm{n}}\left\langle S_{\mathrm{n}}^{\mu}\right\rangle \equiv \Lambda\left\langle\mathrm{N}\left|S^{\mu}\right| \mathrm{N}\right\rangle
$$

where $S^{\mu}$ is the spin operator of the nucleus, we are led to the effective neutralino-nucleus coupling

$$
\mathscr{L}_{\mathrm{A}}=2 \sqrt{2} G_{F} \Lambda\left(\bar{\chi} \gamma_{\mu} \gamma_{5} \chi\right)\left\langle\mathrm{N}\left|S^{\mu}\right| \mathrm{N}\right\rangle
$$


Exercise $\mathbf{n}^{0}$ 1-g - Level [1] : Should the nucleus be a point-like Dirac fermion of spin 1/2, its spin operator would be given by

$$
\left\langle\mathrm{N}\left|S^{\mu}\right| \mathrm{N}\right\rangle \equiv \frac{1}{2}\left(\bar{\Psi}_{\mathrm{N}} \gamma^{\mu} \gamma_{5} \Psi_{\mathrm{N}}\right)
$$

Compute the average over the initial spin states and the sum over the final spin states of the square of the amplitude of the neutralino-nucleus interaction (1.41) and show that

$$
\mathscr{A}=8 \kappa G_{F}^{2} \Lambda^{2} \times \frac{3}{4} .
$$

where the parameter $\kappa=4$ since the neutralino is a Majorana fermion.

Exercise $\mathbf{n}^{0} \mathbf{1 - h}-$ Level [2] : Slightly more involved is the calculation of $\mathscr{A}$ when the nucleus has a spin $J$ not necessarily equal to $1 / 2$. We will still work in the non-relativistic limit where the various space-momenta that come into play in the calculation are negligible with respect to the energies. The nucleus is at rest and the time-component $S^{0}$ of its spin vanishes. Show that

$$
\mathscr{A}=8 \kappa G_{F}^{2} \Lambda^{2} \times \frac{1}{2(2 J+1)} \times \chi_{\mu \nu} \times \mathrm{N}^{\mu \nu}
$$

where the neutralino tensor reads

$$
\chi_{\mu \nu} \equiv \operatorname{Tr}\left\{\left(\frac{P_{2}+m_{\chi}}{2 m_{\chi}}\right) \times \gamma_{\mu} \gamma_{5} \times\left(\frac{P_{1}+m_{\chi}}{2 m_{\chi}}\right) \times \gamma_{v} \gamma_{5}\right\},
$$

whereas the tensor related to the spin of the nucleus is

$$
\mathrm{N}^{\mu v} \equiv \sum_{m_{i}, m_{f}}\left\langle J, m_{f}\left|S^{\mu}\right| J, m_{i}\right\rangle\left\langle J, m_{i}\left|S^{\nu}\right| J, m_{f}\right\rangle .
$$

Compute $\chi_{\mu v}$ and show that the only piece that gives a non-vanishing Lorentz contraction with $\mathrm{N}^{\mu v}$ is

$$
\chi_{\mu \nu} \supset\left\{-\eta_{\mu v}\left(1+\frac{P_{1} \cdot P_{2}}{m_{\chi}^{2}}\right)\right\} \simeq-2 \eta_{\mu v} .
$$

Use the closure relation on the initial spin states of the nucleus to derive

$$
\mathscr{A}=8 \kappa G_{F}^{2} \Lambda^{2} \times \frac{1}{(2 J+1)} \times \sum_{m_{f}}\left\langle J, m_{f}\left|\mathbf{S}^{2}\right| J, m_{f}\right\rangle .
$$

Establish eventually that

$$
\mathscr{A}=8 \kappa G_{F}^{2} \Lambda^{2} \times J(J+1)
$$

We readily conclude from the previous problem that the spin dependent differential cross section may be expressed as

$$
\frac{d \sigma^{\mathrm{SD}}}{d \Omega^{*}}=\frac{2 \kappa}{\pi^{2}} G_{F}^{2} \Lambda^{2} \mu^{2} J(J+1)
$$


where the factor $A^{2} g_{S}^{2}$ of the spin independent cross section (1.8) is now replaced by the factor $8 G_{F}^{2} \Lambda^{2} J(J+1)$. Notice that the scattering of the neutralino in the center of mass frame is still isotropic. Another form for the axial cross section is

$$
\frac{d \sigma^{\mathrm{SD}}}{d \mathbf{q}^{2}}=\frac{2 \kappa}{\pi} \frac{G_{F}^{2}}{\mathbf{V}_{\chi}^{2}} \Lambda^{2} J(J+1)
$$

where $\mathbf{V}_{\chi}$ denotes the neutralino velocity in the laboratory frame. The parameter $\Lambda$ depends on the effective couplings $a_{\mathrm{p}}$ and $a_{\mathrm{n}}$ as well as on the amounts of spin $\left\langle S_{\mathrm{p}}\right\rangle$ and $\left\langle S_{\mathrm{n}}\right\rangle$ carried out respectively by the proton and the neutron groups inside the target nucleus. Reasonably accurate estimates of the latter can be made using the odd-group model [10] which assumes that all the nuclear spin is carried out by the odd group, either the protons or the neutrons, whichever is most unpaired. Thus only one of either $\left\langle S_{\mathrm{p}}\right\rangle$ or $\left\langle S_{\mathrm{n}}\right\rangle$ is non-vanishing whilst the other is equal to zero. In the case of ${ }^{73} \mathrm{Ge}$, the odd-group model gives a very poor estimate in disagreement with the detailed calculations of $[11,12]$. A summary of values for $\left\langle S_{\mathrm{p}}\right\rangle$ and $\left\langle S_{\mathrm{n}}\right\rangle$ is provided in table 2 where the results from the odd-group model are compared to more sophisticated calculations for the few nuclei that have been used in the most recent experiments.

\begin{tabular}{|cccccl|}
\hline \hline \multirow{2}{*}{ Nucleus } & $\left\langle S_{\mathrm{p}}\right\rangle_{\text {OGM }}$ & $\left\langle S_{\mathrm{n}}\right\rangle_{\mathrm{OGM}}$ & $\left\langle S_{\mathrm{p}}\right\rangle$ & $\left\langle S_{\mathrm{n}}\right\rangle$ & Model \\
& & & & & \\
${ }^{19} \mathrm{~F}$ & 0.46 & 0.0 & 0.415 & -0.047 & EOGM1 [10] \\
& & & 0.368 & -0.001 & EOGM2 [10] \\
${ }^{27} \mathrm{Al}$ & 0.25 & 0.0 & -0.343 & 0.030 & Shell model [13] \\
${ }^{29} \mathrm{Si}$ & 0.0 & 0.15 & -0.002 & 0.13 & Shell model [11] \\
${ }^{73} \mathrm{Ge}$ & 0.0 & 0.23 & 0.011 & 0.491 & Shell model [11] \\
& & & 0.030 & 0.378 & Hybrid [12] \\
${ }^{93} \mathrm{Nb}$ & 0.36 & 0.0 & 0.46 & 0.08 & Shell model [14] \\
${ }^{131} \mathrm{Xe}$ & 0.0 & -0.166 & -0.041 & -0.236 & IBFM [15] \\
\hline \hline
\end{tabular}

Table 2: Comparison of odd-group model results with more sophisticated calculations. EOGM is the extended odd-group model whereas IBFM is the interacting-boson-fermion scheme.

A final ingredient needs to be incorporated in the calculation of the spin dependent cross section. The impinging DM particle probes the nuclear structure on a distance

$$
\lambda \sim|\mathbf{q}|^{-1} \sim 1 / \sqrt{2 m_{\mathrm{N}} E_{R}},
$$

which may be smaller than the nucleus size. For a ${ }^{131} \mathrm{Xe}$ nucleus and a recoil energy of $100 \mathrm{keV}$, the diffraction scale is $\sim 1$ fermi and is 5 times smaller than the nuclear radius. A form factor needs to be introduced in the cross section (1.51) which becomes

$$
\frac{d \sigma^{\mathrm{SD}}}{d \mathbf{q}^{2}}=\frac{2 \kappa}{\pi} \frac{G_{F}^{2}}{\mathbf{V}_{\chi}^{2}} \Lambda^{2} J(J+1)\left\{\frac{S(|\mathbf{q}|)}{S(0)}\right\} .
$$

The neutralino-proton and neutralino-neutron couplings can be rearranged into an isoscalar $\left(q_{\mathrm{f}}=a_{\mathrm{p}}+a_{\mathrm{n}}\right)$ and an isovector $\left(a_{1}=a_{\mathrm{p}}-a_{\mathrm{n}}\right)$ part to yield

$$
S(|\mathbf{q}|)=a_{0}^{2} S_{00}(|\mathbf{q}|)+a_{0} a_{1} S_{01}(|\mathbf{q}|)+a_{1}^{2} S_{11}(|\mathbf{q}|) .
$$


The independent form factors $S_{00}(|\mathbf{q}|), S_{01}(|\mathbf{q}|)$ and $S_{11}(|\mathbf{q}|)$ are obtained from detailed nuclear calculations. Parametric fits can be found in [16] where results for ${ }^{73} \mathrm{Ge}[12],{ }^{27} \mathrm{Al}[13]$ and ${ }^{29} \mathrm{Si}$ [11] are featured.

The careful reader will have certainly noticed at this stage that the calculation of the spin dependent interaction cross section between a neutralino and a target nucleus is plagued by large uncertainties at several levels. If neutralinos turn out to be discovered at the LHC, it will be of the utmost importance to reduced these uncertainties in order to make reliable predictions for direct detection and to be able to compare with the accelerator results.
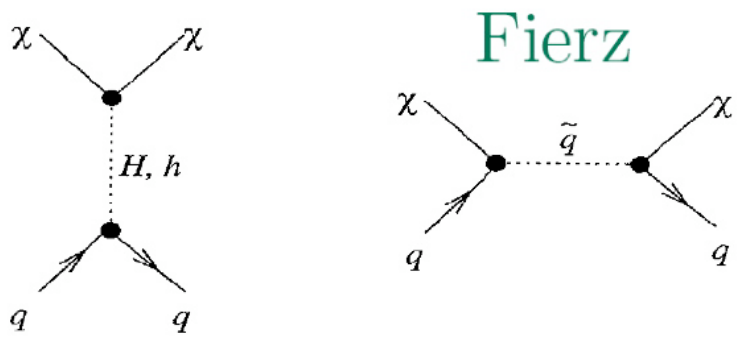

Figure 7: Feynman diagrams contributing to the scalar elastic scattering of neutralinos off quarks and eventually to the spin independent neutralino-nucleus scattering cross section.

Scalar coupling - The microscopic scalar coupling of neutralinos to quarks is given at tree level by a Higgs and a squark exchange as shown in figure 7. Neutralinos couple also to gluons through a quark/squark loop. The tensor couplings $\bar{\chi}\left(i \partial_{\mu} \gamma_{\nu}+i \partial_{\nu} \gamma_{\mu}\right) \chi$ and $\bar{\chi} \partial_{\mu} \partial_{\nu} \chi$ arise finally from twist-two operators. In order to simplify the discussion, we will concentrate here only on the tree level diagrams with Yukawa-type couplings $\lambda_{q} \propto m_{q}$.

The first step in the calculation of the spin independent scattering cross section is to evaluate the neutralino-nucleon effective coupling

$$
\mathscr{L}_{\mathrm{S}}=\sum_{q} \frac{\lambda_{q}}{m_{q}}\left\langle n\left|m_{q} \bar{q} q\right| n\right\rangle(\bar{\chi} \chi)
$$

where $n$ denotes either the proton or the neutron. The matrix elements of the three light quarks can be derived [17] from the pion-nucleon sigma term

$$
\sigma_{\pi n}=\frac{m_{\mathrm{u}}+m_{\mathrm{d}}}{2}\langle n|\overline{\mathrm{u}} \mathrm{u}+\overline{\mathrm{d}} \mathrm{d}| n\rangle,
$$

the fraction contributed by the strange quark to the content of the nucleon

$$
y=2 \frac{\langle n|\overline{\mathrm{s}} \mathrm{s}| n\rangle}{\langle n|\overline{\mathrm{u}} \mathrm{u}+\overline{\mathrm{d}} \mathrm{d}| n\rangle},
$$

and the mass ratio

$$
r=\frac{2 m_{\mathrm{s}}}{m_{\mathrm{u}}+m_{\mathrm{d}}} .
$$


Exercise $\mathbf{n}^{0}$ 1-i - Level [1] : Assume that isospin invariance holds between the up and down quarks in the previous relations and derive

$$
m_{\mathrm{u}}\langle n|\overline{\mathrm{u}} \mathrm{u}| n\rangle=m_{\mathrm{d}}\langle n|\overline{\mathrm{d}} \mathrm{d}| n\rangle=\frac{1}{2} \sigma_{\pi n},
$$

as well as

$$
m_{\mathrm{s}}\langle n|\overline{\mathrm{s}} \mathrm{s}| n\rangle=\frac{r y}{2} \sigma_{\pi n} .
$$

Does the latter expression still hold if the assumption of isospin invariance between the up and down quarks is relaxed?

The values of these three parameters are affected by large uncertainties and depend on the method followed to evaluate them. To commence, the pion-nucleon sigma term $\sigma_{\pi n}$ can be derived phenomenologically from measurements of the pion-nucleon scattering cross section. However, the customary procedure is rather involved and goes through several steps [17].

(i) Experimental data of low-energy pion-nucleon scattering can be converted through phase-shift analysis and dispersion relations into

$$
\Sigma_{\mathrm{CD}} \equiv \Sigma\left(t=2 m_{\pi}^{2}\right) \equiv f_{\pi}^{2} \bar{D}^{+}\left(s=u=m_{n}^{2}, t=2 m_{\pi}^{2}\right),
$$

where $s, u$ and $t$ are standard Mandelstam variables, $m_{\pi}$ is the pion mass, $f_{\pi}$ is the pion-decay constant whereas $\bar{D}^{+}$stands for the pion-nucleon isoscalar scattering D-amplitude with the pseudovector Born term substracted and calculated at the so-called Cheng-Dashen (CD) point. (ii) Up to terms of order $\sim 1 \mathrm{MeV}$ which may be safely neglected, this quantity is equal to

$$
\Sigma_{\mathrm{CD}} \simeq \sigma_{\pi n}\left(t=2 m_{\pi}^{2}\right)
$$

where

$$
\sigma_{\pi n}\left\{t=\left(p_{f}-p_{i}\right)^{2}\right\} \equiv\left\langle n, p_{f}\left|\left\{\frac{m_{\mathrm{u}}+m_{\mathrm{d}}}{2}(\overline{\mathrm{u}} \mathrm{u}+\overline{\mathrm{d}} \mathrm{d})\right\}\right| n, p_{i}\right\rangle
$$

is the nucleon scalar form factor.

(iii) The evolution of $\sigma_{\pi n}(t)$ as a function of the momentum transfer from $t=2 m_{\pi}^{2}$ down to $t=0$ leads to the pion-nucleon sigma term

$$
\left\{\sigma_{\pi n} \equiv \sigma_{\pi n}(t=0)\right\}=\sigma_{\pi n}\left(t=2 m_{\pi}^{2}\right)-\Delta \sigma .
$$

The determination of $\Sigma_{\mathrm{CD}}$ and $\Delta \sigma$ are plagued by considerable uncertainties. In [17], the range extending from 56 up to $72 \mathrm{MeV}$ is proposed for $\Sigma_{\mathrm{CD}}$. Dispersion relation techniques [18] provide an estimate of $\sim 15 \mathrm{MeV}$ for $\Delta \sigma$ which is twice as large as the result from chiral perturbation theory at leading order [19]. Lattice calculations yield also a value of $\Delta \sigma=6.6 \pm 0.6 \mathrm{MeV}$ which is clearly in disagreement with dispersion relation techniques. Combining these values for $\Sigma_{\mathrm{CD}}$ and $\Delta \sigma$ leads to a conservative range for the pion-nucleon sigma term $\sigma_{\pi n}$ which extends from 41 up to $65 \mathrm{MeV}$.

Then, the strange quark fraction $y$ is related to $\sigma_{\pi n}$ through the identity

$$
y=1-\frac{\sigma_{0}}{\sigma_{\pi n}},
$$


where the quantity $\sigma_{0}$ is defined as

$$
\sigma_{0}=\frac{m_{\mathrm{u}}+m_{\mathrm{d}}}{2}\langle n|\overline{\mathrm{u}} \mathrm{u}+\overline{\mathrm{d}} \mathrm{d}-2 \overline{\mathrm{s}} \mathrm{s}| n\rangle .
$$

As discussed in [17], $\sigma_{0}$ is related to the magnitude of the $\mathrm{SU}(3)$ flavor symmetry breaking and may be derived either from the octet baryon masses which yield a value of $33 \mathrm{MeV}$ [20] or with chiral perturbation theory for which $\sigma_{0}=35 \pm 5 \mathrm{MeV}$ [19] or $36 \pm 7 \mathrm{MeV}$ [21].

The ratio $r=2 m_{\mathrm{s}} /\left(m_{\mathrm{u}}+m_{\mathrm{d}}\right)$ is the last ingredient needed to evaluate the matrix element of the strange quark $\left\langle n\left|m_{\mathrm{s}} \overline{\mathrm{s}} \mathrm{s}\right| n\right\rangle$. Chiral perturbation theory gives at the lowest order - though corrected for electromagnetic effects - the mass ratios $m_{\mathrm{u}} / m_{\mathrm{d}}$ and $m_{\mathrm{s}} / m_{\mathrm{d}}$ in terms of the physical masses of the $K$ mesons and lead to the canonical value [22]

$$
r \simeq 26 \text {. }
$$

This value is modified by next-to-leading order contributions in the chiral expansion. QCD sum rules and lattice simulations can alternatively provide estimates for the quark masses directly and not only for their ratios. Borrowing the values from [23] where $m_{\mathrm{s}}(1 \mathrm{GeV})=175 \pm 25 \mathrm{MeV}$ and $\left(m_{\mathrm{u}}+m_{\mathrm{d}}\right)(1 \mathrm{GeV})=12 \pm 2.5 \mathrm{MeV}$ leads to the ratio

$$
r=31 \pm 11
$$

The lack of determination of the pion-nucleon sigma term $\sigma_{\pi n}$, of the strange fraction $y$ and of the mass ratio $r$ implies a significant uncertainty in the calculation of the effective neutralino-nucleon coupling. The matrix element $\left\langle n\left|m_{q} \bar{q} q\right| n\right\rangle$ describes the amount of mass carried out by each light quark species $q$ inside the nucleon $n$ and may be actually parameterized as

$$
\left\langle n\left|m_{q} \bar{q} q\right| n\right\rangle \equiv m_{n} f_{T q}^{(n)} .
$$

The mass fractions $f_{T q}^{(n)}$ are listed in table 3. The contribution of the strange quark is the most uncertain since it varies from 0.08 up to 0.46 depending on the analysis.

\begin{tabular}{|lccccc|}
\hline \hline Nucleon & $f_{T \mathrm{u}}$ & $f_{T \mathrm{~d}}$ & $f_{T \mathrm{~s}}[24]$ & $f_{T \mathrm{~s}}[25]$ & $f_{T \mathrm{~s}}[20,26]$ \\
$\mathrm{n}$ & 0.023 & 0.034 & 0.08 & 0.14 & 0.46 \\
$\mathrm{p}$ & 0.019 & 0.041 & 0.08 & 0.14 & 0.46 \\
\hline \hline
\end{tabular}

Table 3: Estimates for the quark mass fractions $f_{T q}$ inside the neutron and the proton. The up and down quark contributions are obtained from [26] whereas the value for the strange quark is subject to large variations depending on the author.

In order to complete the calculation, two key ingredients are necessary.

- The nucleon mass is given by the trace of the QCD energy-momentum tensor

$$
m_{n}=\left\langle n\left|\left\{\Theta_{\mu}^{\mu} \equiv \sum_{\mathrm{u}, \mathrm{d}, \mathrm{s}} m_{q} \bar{q} q+\sum_{\mathrm{c}, \mathrm{b}, \mathrm{t}} m_{Q} \bar{Q} Q-\frac{7 \alpha_{\mathrm{S}}}{8 \pi} G G\right\}\right| n\right\rangle .
$$

- In the limit where the quarks $Q$ are heavy, their contributions to the nucleon mass occur through an anomalous loop diagram [27] and can be expressed in terms of the gluonic content as

$$
\left\langle n\left|m_{Q} \bar{Q} Q\right| n\right\rangle \equiv-\frac{2 \alpha_{\mathrm{S}}}{24 \pi}\langle n|G G| n\rangle,
$$


where $\alpha_{\mathrm{S}}$ is the strong coupling constant.

Exercise $\mathbf{n}^{0} \mathbf{1}-\mathbf{j}$ - Level [1] : Show that the neutralino-nucleon scalar interaction may be effectively described by the Lagrangian

$$
\mathscr{L}_{\mathrm{S}}=f_{(n)}(\bar{n} n)(\bar{\chi} \chi)
$$

where the couplings $f_{(n)}$ are given by

$$
f_{(n)}=m_{n}\left\{\sum_{\mathrm{u}, \mathrm{d}, \mathrm{s}} \frac{\lambda_{q}}{m_{q}} f_{T q}^{(n)}+\frac{2}{27}\left(1-\sum_{\mathrm{u}, \mathrm{d}, \mathrm{s}} f_{T q}^{(n)}\right) \sum_{\mathrm{c}, \mathrm{b}, \mathrm{t}} \frac{\lambda_{Q}}{m_{Q}}\right\} .
$$

The effective neutralino-nucleon coupling needs now to be embedded inside the nuclear state $|\mathrm{N}\rangle$ to yield the effective neutralino-nucleus scalar interaction. This step is straightforward since the operators $(\overline{\mathrm{p}} \mathrm{p})$ and $(\overline{\mathrm{n}} \mathrm{n})$ count respectively the number of protons $(\mathrm{Z})$ and neutrons $(\mathrm{N} \equiv \mathrm{A}-\mathrm{Z})$ inside the target nucleus and is much simpler than in the axial case where involved nuclear calculations were necessary. This leads to the effective Lagrangian

$$
\mathscr{L}_{\mathrm{S}}=\left\{Z f_{\mathrm{p}}+(A-Z) f_{\mathrm{n}}\right\}(\bar{\chi} \chi)\left(\bar{\Psi}_{\mathrm{N}} \Psi_{\mathrm{N}}\right)
$$

where the factor $A g_{S}$ of the naïve expression (1.3) has been replaced now by the combination

$$
A g_{S} \rightarrow Z f_{\mathrm{p}}+(A-Z) f_{\mathrm{n}}
$$

The proton $f_{\mathrm{p}}$ and neutron $f_{\mathrm{n}}$ scalar couplings are defined by relation (1.73). We readily infer the differential spin independent neutralino-nucleus elastic scattering cross section

$$
\frac{d \sigma^{\mathrm{SI}}}{d \Omega^{*}}=\frac{\kappa}{4 \pi^{2}} \mu^{2}\left\{Z f_{\mathrm{p}}+(A-Z) f_{\mathrm{n}}\right\}^{2} .
$$

This expression may be cast into the same shape as relation (1.51)

$$
\frac{d \sigma^{\mathrm{SI}}}{d \mathbf{q}^{2}}=\frac{\kappa}{4 \pi} \frac{1}{\mathbf{V}_{\chi}^{2}}\left\{Z f_{\mathrm{p}}+(A-Z) f_{\mathrm{n}}\right\}^{2}
$$

For large enough transfer momenta $\mathbf{q}$, the neutralino undergoes a diffraction on the nucleus and the scattering amplitude is modulated by the form factor $F(|\mathbf{q}|)$ so that the elastic spin independent interaction cross section becomes

$$
\frac{d \sigma^{\mathrm{SI}}}{d \mathbf{q}^{2}}=\frac{\kappa}{4 \pi} \frac{1}{\mathbf{V}_{\chi}^{2}}\left\{Z f_{\mathrm{p}}+(A-Z) f_{\mathrm{n}}\right\}^{2} F^{2}(|\mathbf{q}|)
$$

Notice that the square of the form factor $F^{2}(|\mathbf{q}|)$ is here the scalar equivalent of the ratio $S(|\mathbf{q}|) / S(0)$ which damps out the axial cross section (1.53). The form factor of the spin independent case is associated to the nucleon number operators $(\overline{\mathrm{p}} \mathrm{p})$ and $(\overline{\mathrm{n}} \mathrm{n})$ and is simply the Fourier transform of the nucleon density. The latter is well-determined and several forms for $F(|\mathbf{q}|)$ are available. 
The short and long-dashed green curve of figure 5 features the effect on the exclusion plot for

${ }^{73} \mathrm{Ge}$ of the exponential form factor $[28,29]$

$$
F(|\mathbf{q}|) \equiv F\left(E_{R}\right)=\exp \left(-E_{R} / 2 Q_{0}\right)
$$

The nuclear coherence energy

$$
Q_{0}=\frac{3}{2 m_{N} R_{\mathrm{N}}^{2}}
$$

depends on the nuclear radius $R_{\mathrm{N}}$ which is defined here as

$$
R_{\mathrm{N}}=1 \text { fermi } \times\left(0.3+0.89 A^{1 / 3}\right)
$$

Except for very light nuclei for which the previous exponential form factor applies well, a universal parameterization is provided by $[30,31]$

$$
F(|\mathbf{q}|)=3 \frac{j_{1}\left(|\mathbf{q}| r_{0}\right)}{|\mathbf{q}| r_{0}} \exp \left\{-\frac{1}{2} s^{2} \mathbf{q}^{2}\right\}
$$

where $j_{1}\left(|\mathbf{q}| r_{0}\right)$ is the spherical Bessel function of index 1. The thickness parameter of the nuclear surface $s \simeq 1$ fermi is related to the radius $r_{0}$ through

$$
r_{0}^{2}=R_{\mathrm{N}}^{2}-5 s^{2}
$$

where the nuclear radius is now defined by

$$
R_{\mathrm{N}}=1.2 \text { fermi } \times A^{1 / 3} \text {. }
$$

The total neutralino-nucleus elastic scattering cross section is the sum of the axial and scalar parts

$$
\frac{d \sigma^{\mathrm{tot}}}{d \mathbf{q}^{2}}=\frac{d \sigma^{\mathrm{SD}}}{d \mathbf{q}^{2}}+\frac{d \sigma^{\mathrm{SI}}}{d \mathbf{q}^{2}}
$$

To conclude this section, let us insist on the very large uncertainties which affect the determination of the effective axial and scalar coupling parameters coming into play in the neutralino-nucleon and neutralino-nucleus scattering amplitude.

\subsection{The experimental endeavors and achievements}

Detecting galactic neutralinos through their impact on the nuclei of a terrestrial instrument is a real challenge. The detector must be sensitive to an energy deposition in the keV range. The usual calorimetric technique is based on the measurement of the temperature increase induced by the energy deposited by a WIMP-nucleus collision. This increase is all the larger as the heat capacity of the material is low, hence the necessity for bolometers to be operated at very low temperatures around a few tens of a milliKelvin. Even so, the temperature increase amounts only to a microkelvin. Other techniques have been developed since the eighties. Inside a semiconductor like silicon or germanium, the WIMP-nucleus collision generates electron-hole pairs which are collected by an electric field. The conductivity of the material increases sharply during an event hence the possibility to detect the passage of WIMPs through their ionized imprints. The third 

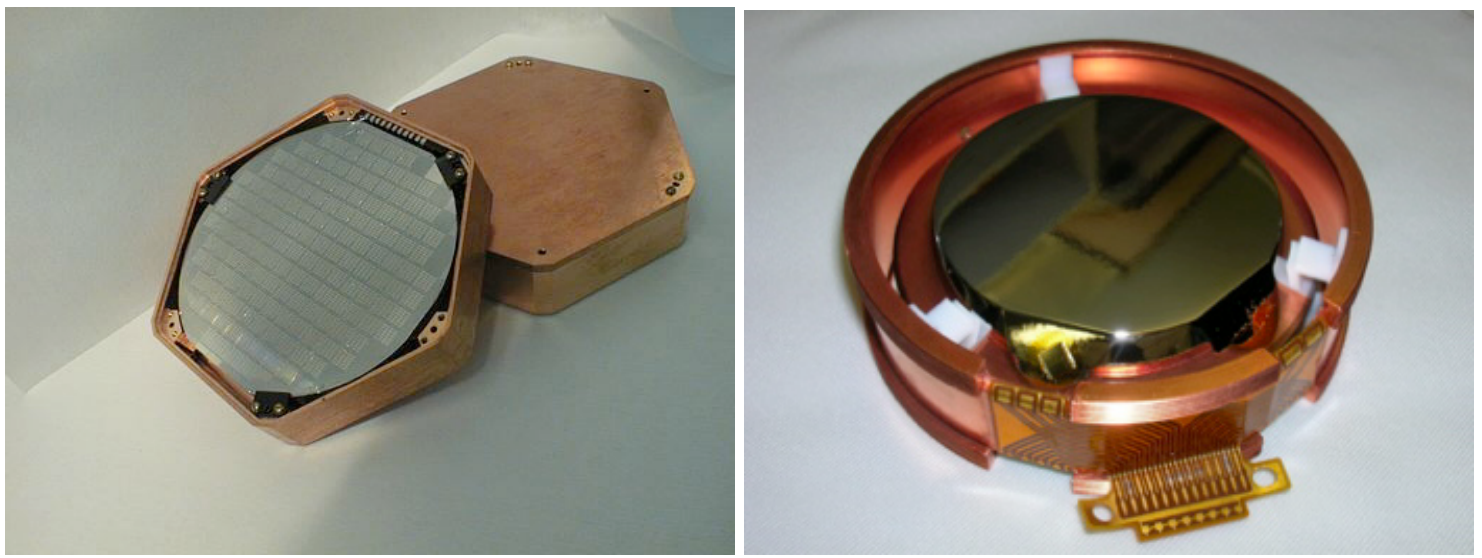

Figure 8: The left panel features a closeup of a ZIP detector in its mount. Such a Si detector was operated in the 1998 run of the CDMS experiment. The photolithographically-fabricated thin film on the surface is the phonon sensor which measures the heat deposition. Silicon and germanium ZIPs, weighing $100 \mathrm{~g}$ and $250 \mathrm{~g}$ respectively, are used in CDMS II runs in the Soudan mine. A germanium detector of the Edelweiss II collaboration is presented for comparison in the right panel. Pictures from [34] and [35].

important technique is based on the scintillation of atoms as they are struck by impinging DM particles. The recoil nucleus interacts with the surrounding medium to produce photons which are subsequently amplified by a photomultiplier and converted into an electrical output signal. These direct detection techniques may be used in association with each other as featured in the triangle of figure 10. Note also that with a collision rate as small as $\sim 0.01-0.1 \mathrm{event} \mathrm{kg}^{-1} \mathrm{day}^{-1}$, large instruments are mandatory. An exposure of 1 ton $\times 10$ days is required to reach the zeptobarn limit. Cooling down such a mass down to a temperature of $\sim 0.01 \mathrm{~K}$ is in itself an adventure. Finally,
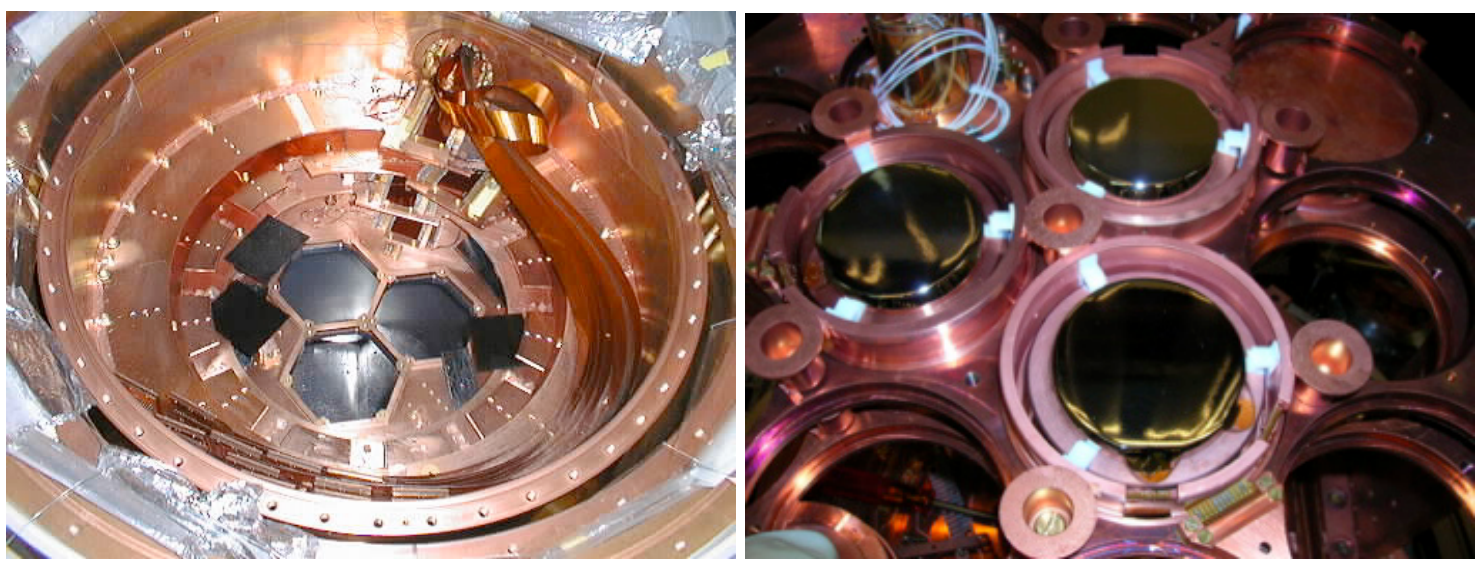

Figure 9: The left panel is a view of the inner layers of the CDMS II cryostat. The ZIP detectors of the previous figure are mounted inside the hexagonal holes and are operated at $10 \mathrm{mK}$. The surrounding layers are higher temperature stages of the cryostat which is constructed entirely of radiopure copper to provide a low-radioactivity environment. The grey-black layer is a shield made of lead recovered from the ballast of a 18th-century French ship. The age of this lead ensures that the radioisotopes most worrying have decayed away. In the right panel, the bolometer towers of the Edelweiss II collaboration are being installed. Pictures from [34] and [35]. 
the signal is buried inside an enormous background which needs to be entirely removed. To get an idea, the potassium ${ }^{40} \mathrm{~K}$ et carbon ${ }^{14} \mathrm{C}$ of a human body generate 8,000 decays each second to be compared to a single WIMP event every day or so. The level of sensitivity which is required in direct detection is outrageously high. The fact that experimentalists have basically succeeded in reaching it is already a great success.

\section{Direct Detection Techniques}

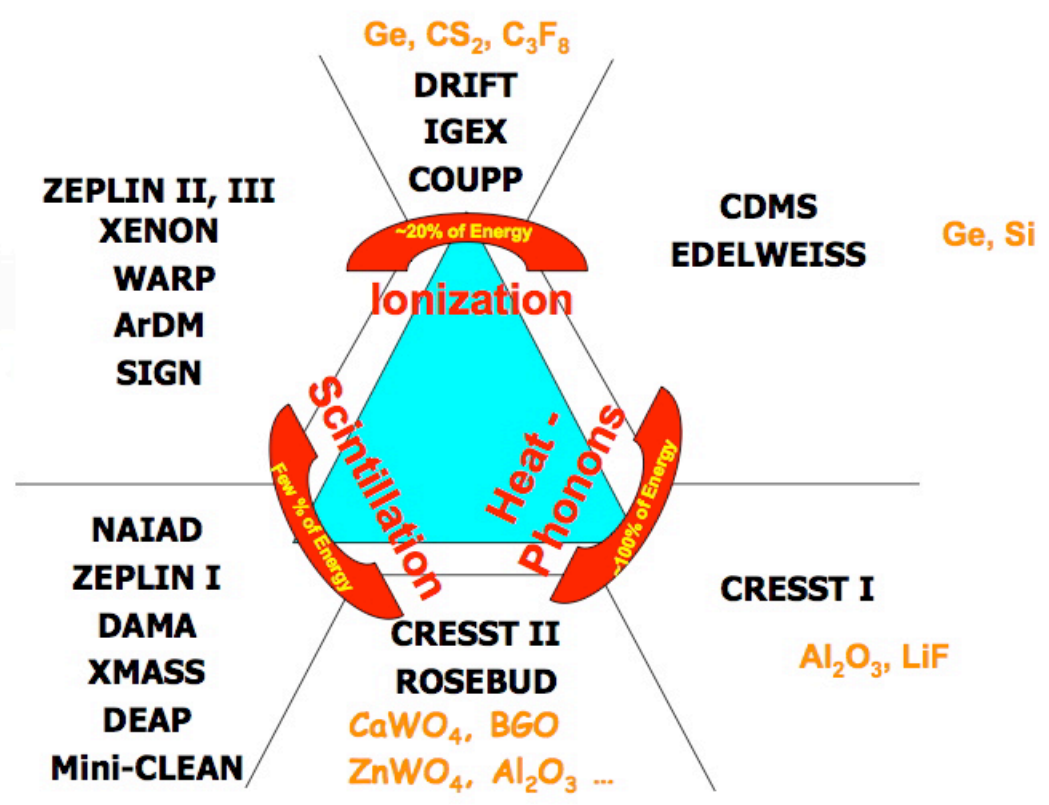

Figure 10: Ionization, scintillation and bolometry - called here heat or phonons - are the three cornerstones of direct detection. They are featured in this diagram so as to form a triangle. These techniques can also be used in association in order to discriminate between several kinds of background. Figure from [33].

Background suppression is a key issue for direct detection experiments. The protection against the surrounding radioactivity has been a central preoccupation and lots of efforts have been devoted. - Cosmic rays - One of the main component of the radioactive background is generated by cosmic rays. High-energy protons and nuclei impinge on the upper atmosphere where they initiate showers of particles heading toward the Earth surface. At sea level, muons are the most numerous charged particles with a mean ground energy of $4 \mathrm{GeV}$ and a flux of $70 \mathrm{~m}^{-2} \mathrm{~s}^{-1} \mathrm{sr}^{-1}$ above $1 \mathrm{GeV}$. The solution to get rid of this background consists in going deep underground. Direct detection experiments are actually installed inside old mines or tunnels. The Soudan Underground Laboratory [36] is located in the Soudan Underground Mine State Park at a depth of 710 meters. The amount of rock above the CDMS II experiment acts as a water shield whose thickness would be 2.1 kilometers. Consequently, the muon flux is reduced by a factor of $10^{5}$. The French Laboratoire Souterrain de Modane [37] sits at the French-Italian border inside the Fréjus tunnel. With a depth of 1,760 meters of rock - 4.6 kilometers of water equivalent - the muon flux at the site is only of 4 particles $\mathrm{m}^{-2}$ day $^{-1}$. The largest underground laboratory [38] is operated in the Gran Sasso highway tunnel connecting L'Aquila to Teramo about 120 kilometers from Roma. Three main 


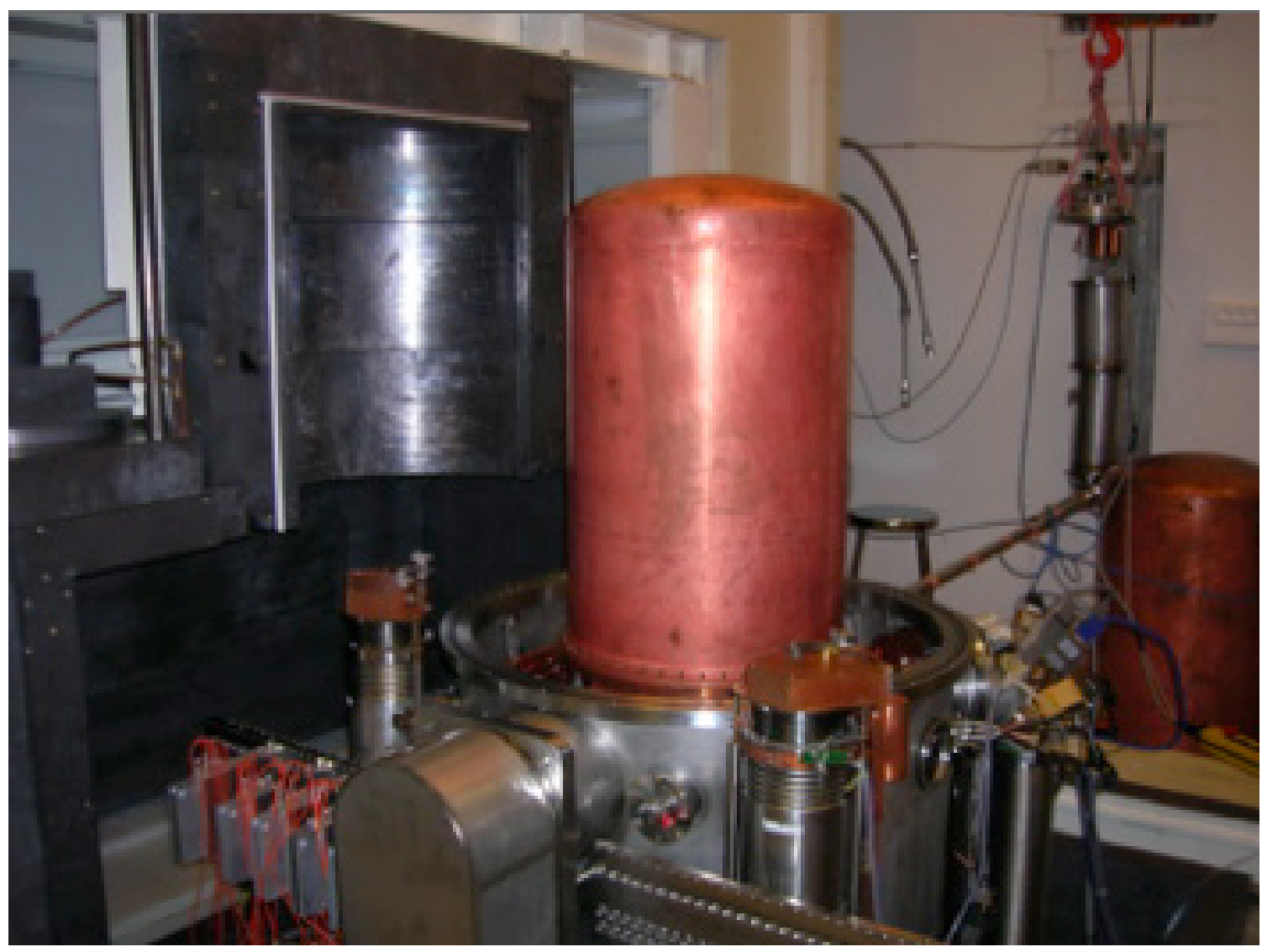

Figure 11: The detectors of the Edelweiss II experiment are installed inside this large copper cryostat and are operated at a temperature of $20 \mathrm{mK}$. The cryostat itself is protected against the radioactive background by the black lead shield which sits behind. Picture from [35].

halls have been excavated at a depth of 1,400 meters that translate into 3.8 kilometers of water equivalent. With a reduction by a factor of $\sim 10^{6}$ with respect to the surface, the muon flux amounts to 24 particles $\mathrm{m}^{-2}$ day $^{-1}$.

- Underground radioactivity - Uranium and thorium are naturally present in the rock of the cavity. Other radioactive species are also found in the materials inside the laboratory. The U-Th disintegration chain generates photons and neutrons. A few muons still make it down to the site. Energetic muon interactions with the surrounding rock and with the materials of the experiment produce neutrons in the $\mathrm{MeV}-\mathrm{GeV}$ range. Detectors are protected against the $\gamma$-ray background by copper or lead shields. Because freshly extracted lead contains the radioactive ${ }^{210} \mathrm{~Pb}$ isotope, ancient lead is preferred such as roman lead in the case of Edelweiss or lead taken from a sunken galleon for CDMS. The lead castle of Edelweiss II has a weight of 35 tons - see figure 11. Neutrons with an energy in the range from $100 \mathrm{keV}$ to $10 \mathrm{MeV}$ can induce 1 to $200 \mathrm{keV}$ nuclear recoils. They can be efficiently stopped by polyethylene or water. In the Edelweiss I experiment for instance, a layer of 20 centimeters of paraffin was enough to reduce the neutron flux by a factor of $10^{3}$. The second stage of the experiment makes use of a 50 centimeters thick polyethylen shield whose weight amounts to 30 tons. In spite of these passive shields, several experiments are still sensitive 
to the neutrons generated by the high-energy muons penetrating the rock down to the site and colliding with the lead shield of the detector. The counteraction consists in wrapping the experiment with a muon veto counter so that data acquired during a muon crossing are substracted.

- Natural radioactivity of the detector - Because the materials used in the construction of the experiment are naturally radioactive - like electric connectors - they must be drastically selected so as to decrease the corresponding background. Ancient lead is one particular example among the many radio-nuclides which are naturally found in most ores. Special care is mandatory in the production of materials in order to reduce their content of $\mathrm{U}$, Th and $\mathrm{K}$ contaminants. Cosmic ray activation is also a problem. Primary neutrons and muons interact with target nuclei at the Earth surface to produce terrestrial cosmogenic nuclides. The primary nuclear processes by which cosmogenic nuclides are produced are spallation, muon capture and neutron activation. Materials should be stored deep underground as soon as they are manufactured. Note that germanium detectors have been developed to select the low radioactive materials of Edelweiss II. A final threat is radon, a noble gas which is produced in the decay chains of uranium and thorium. In underground laboratories, radon is a very important source of surface contamination and almost all the sites are equipped with a radon trapping system. In addition, nitrogen is flushed in most of the experiments near the detector volume in order to reduce the remaining radon contamination. Finally, to avoid the radon daughters which may be embedded inside the materials of the experiment, the inner parts of the detector are assembled and stored underground, in a clean room with low radon contamination and dust.

However, in spite of all the precautions taken in order to shield the detector against undesirable radiations, an irreducible background of photons, electrons and neutrons will still make it down to the heart of the instrument. That is why most of the experiments combine now different detection techniques. This allows to discriminate between the various species that penetrate at the core of the apparatus. Incoming photons and beta radiation transfer energy to the electrons of the material, strip them off their atoms and induce a sizable level of ionization. On the contrary, neutrons just like WIMPs collide only upon nuclei and leave an imprint mostly in the form of phonon energy though some ionization is still present. Experiments like CDMS or Edelweiss utilize germanium or silicon and are based on the simultaneous detection of the ionization and phonon energies. Whatever the type of recoil, the energy $E_{\mathrm{R}}$ deposited inside the detector is partially converted into $N_{Q}$ electronhole pairs which subsequently drift inside the crystal toward the surface where they are collected by electrodes. An electric field $E$ of a few volts/cm is established across the semiconductor by a bias voltage $V_{\mathrm{b}}$. When neutralization is complete, the portion of the deposited energy that has been initially converted into ionization is poured back in the phonon system whose energy $E_{\mathrm{p}}$ comprises then the energy $E_{\mathrm{R}}$ left by the impinging particle. The work done by the electric field in drifting the electrons and holes across the crystal is also converted into additional phonons. This so-called Neganov-Trofimov-Luke effect $[39,40]$ results into an increase of the phonon energy which may now be expressed as

$$
E_{\mathrm{P}}=E_{\mathrm{R}}+\sum_{q} e E d_{q}
$$

where $d_{q}$ denotes the distance over which the charge $q$ has been drifting before hitting an electrode. 
Exercise $\mathbf{n}^{0} \mathbf{1 - k}-$ Level [1] : Each electron is associated to a hole which is collected at the opposite electrode. Show then that the phonon energy may be expressed as

$$
E_{\mathrm{P}}=E_{\mathrm{R}}+N_{\mathrm{Q}} e V_{\mathrm{b}} .
$$

The ionization measures the number $N_{\mathrm{Q}}$ of electron-hole pairs left by the incoming particle. By convention, the ionization energy $E_{\mathrm{Q}}$ is defined as the energy $E_{\mathrm{R}}$ deposited by an electron recoil producing the same number $N_{\mathrm{Q}}$ of electron-hole pairs and assuming a perfect collection of charges by the electrodes. The ionization energy $E_{\mathrm{Q}}$ is therefore referred to as the energy deposited by the equivalent electron recoil and is expressed in units of $\mathrm{keV}$ electron-equivalent or keVee. The formation of an electron-hole pair requires an average electron recoil energy $\varepsilon$ of $3 \mathrm{eV}$ in germanium and of $3.8 \mathrm{eV}$ in silicon. This electron-equivalent ionization energy $\varepsilon$ is the scaling factor between the number $N_{\mathrm{Q}}$ of electron-hole pairs and the ionization energy $E_{\mathrm{Q}}$ hence

$$
E_{\mathrm{Q}}=N_{\mathrm{Q}} \varepsilon
$$

An event characterized by the ionization energy $E_{\mathrm{Q}}$ and the phonon energy $E_{\mathrm{P}}$ is thus associated to the deposited energy

$$
E_{\mathrm{R}}=E_{\mathrm{P}}-\frac{e V_{\mathrm{b}}}{\varepsilon} E_{\mathrm{Q}} .
$$

The ionization yield is defined as the dimensionless ratio $y=E_{\mathrm{Q}} / E_{\mathrm{R}}$ of ionization energy to recoil energy. By definition of $E_{\mathrm{Q}}$, this ratio is unity for electron recoils. Measurements indicate a value of a third for nuclear recoils as featured by the green band in the left panel of figure 12. A few ZIP detectors of the CDMS II experiment have been calibrated with photon and neutron sources in order to test the efficiency of the rejection of electron recoils. The ionization yield $y$ alone allows only a rough discrimination between electron and nuclear recoils. The problem arises from the existence of events with intermediate ionization yields. Calibration using a ${ }^{133}$ Ba gamma ray source actually brings to light electron recoils with small ionization yield of order 0.1 to 0.8 . Such events could be misinterpreted as nuclear recoils should the yield parameter $y$ be the only indicator. The black crosses of the right panel of figure 12 are now understood as electron recoils taking place close to the surface of the crystal. In this region, charge collection by the electrodes is less efficient than in the bulk of the material because the electron-hole recombination becomes competitive with the drag of the electric field. Electron recoils produce electron-hole pairs which drift in the crystal toward the electrodes. As they collide on the lattice, these charges generate ballistic phonons which move at the speed of sound in the material - a few millimeters per microsecond in germanium and silicon. The charges however are collected in less than a microsecond. The phonon signal is thus delayed with respect to the ionization pulse. In the case of electron recoils, the delay time of the phonon peak is of order 5 miroseconds. A nuclear recoil is a direct impact on the lattice and generates high-frequency phonons whose propagation through the crystal is quasi-diffusive hence a smaller speed of order a third of the sound speed. The phonon signal of a nuclear recoil should therefore be detected on average 10 to 15 microseconds after the ionization pulse. The blue open circles in the right panel of figure 12 have on average larger phonon time delays than the red dots. 

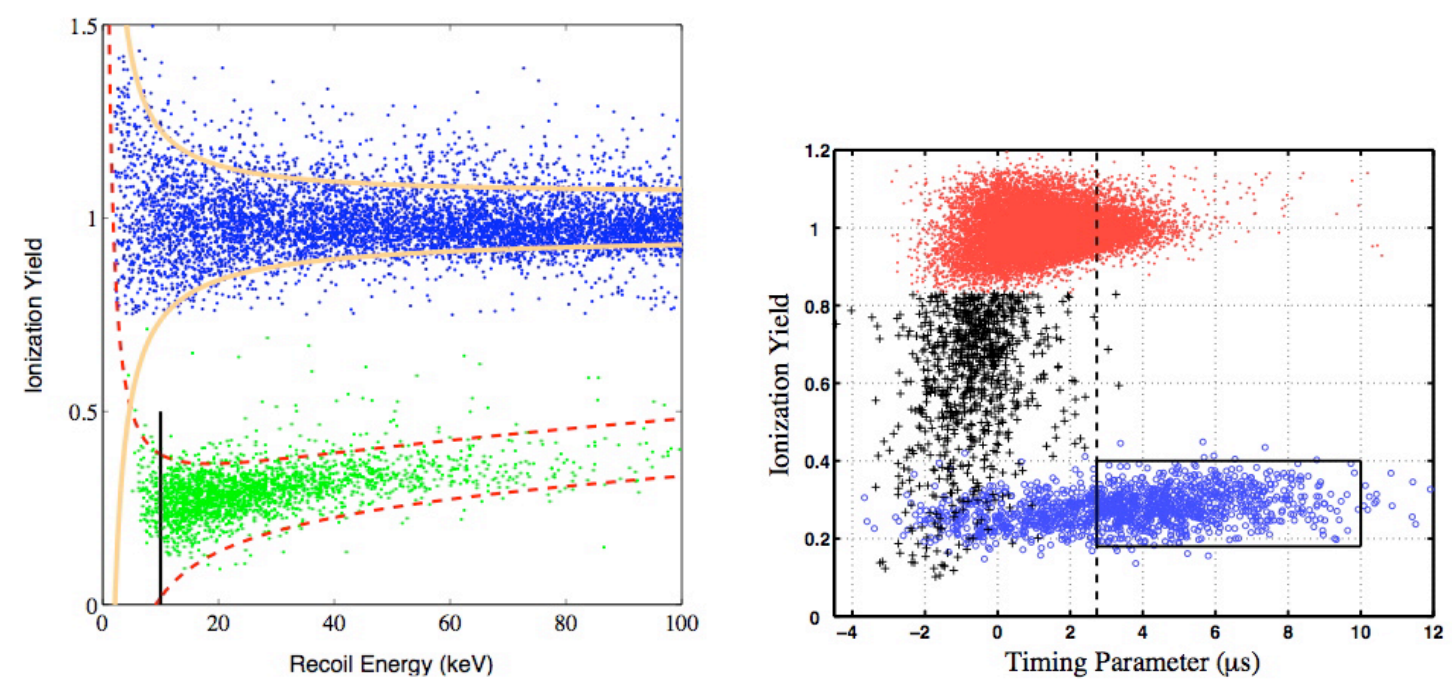

Figure 12: A few ZIP detectors of the CDMS II experiment have been calibrated with photon and neutron sources. In the left panel, the ionization yield $y$ is plotted as a function of the recoil energy $E_{\mathrm{R}}$ in the case of a ${ }^{252} \mathrm{Cf}$ gamma ray and neutron source. Figure from [41]. In the right panel, the ionization yield of the calibration events are featured as a function of the timing parameter, a combination between the phonon time delay relative to the ionization signal and the phonon pulse rise time. The red dots correspond to the electron recoils - generated by a ${ }^{133} \mathrm{Ba}$ photon source - that take place in the bulk of the crystal. The black crosses are low ionization yield ${ }^{133} \mathrm{Ba}$ photon collisions and may mimic nuclear recoils. Because of their small timing parameters, these events are identified as electron recoils occurring close to the surface of the crystal. The blue open circles are true nuclear recoils induced by the neutrons from ${ }^{252} \mathrm{Cf}$. Figure from [32]. The simultaneous measurement of the ionization yield and timing parameter for each event allows to disentangle the nuclear recoils from the dominant background of electron recoils.

They are also fairly scattered as a result of the presence of ballistic phonons whose fraction is smaller though than for electron recoils. The simultaneous determination of the ionization yield $y$ and timing parameter for each event is a powerful tool to reject the abundant background of electron events and to extract the nuclear recoils alone.

Scintillation and ionization are jointly used in XENON10, an experiment utilizing liquid and gaseous xenon as the detecting medium for WIMPs. The detector is presented in figure 13. In spite of its small size, it has provided the best limit so far on the spin independent WIMP-nucleon cross section [5]. The diagram of figure 14 indicates how the detector operates. Events taking place inside liquid xenon generate a first pulse of light $S_{1}$ which is preferentially reflected downward at the liquid-gas interface. This direct scintillation is predominantly collected by an array of photomultiplier tubes (PMTs) located at the bottom of the tank. Some ionization is also produced. The free electrons stripped from the xenon atoms of the liquid phase drift upward. They are dragged by the vertical electric field established in the vessel and move toward the anode located above the gaseous phase. Extracting the ionization electrons from liquid xenon is a major development in this field of research. As the electrons accelerate in the gas gap, they interact with xenon atoms and produce a second pulse of light $\mathrm{S}_{2}$ whose intensity is proportional to the ionization. This secondary scintillation is collected by the top array of PMTs. The horizontal position of the initial recoil is 

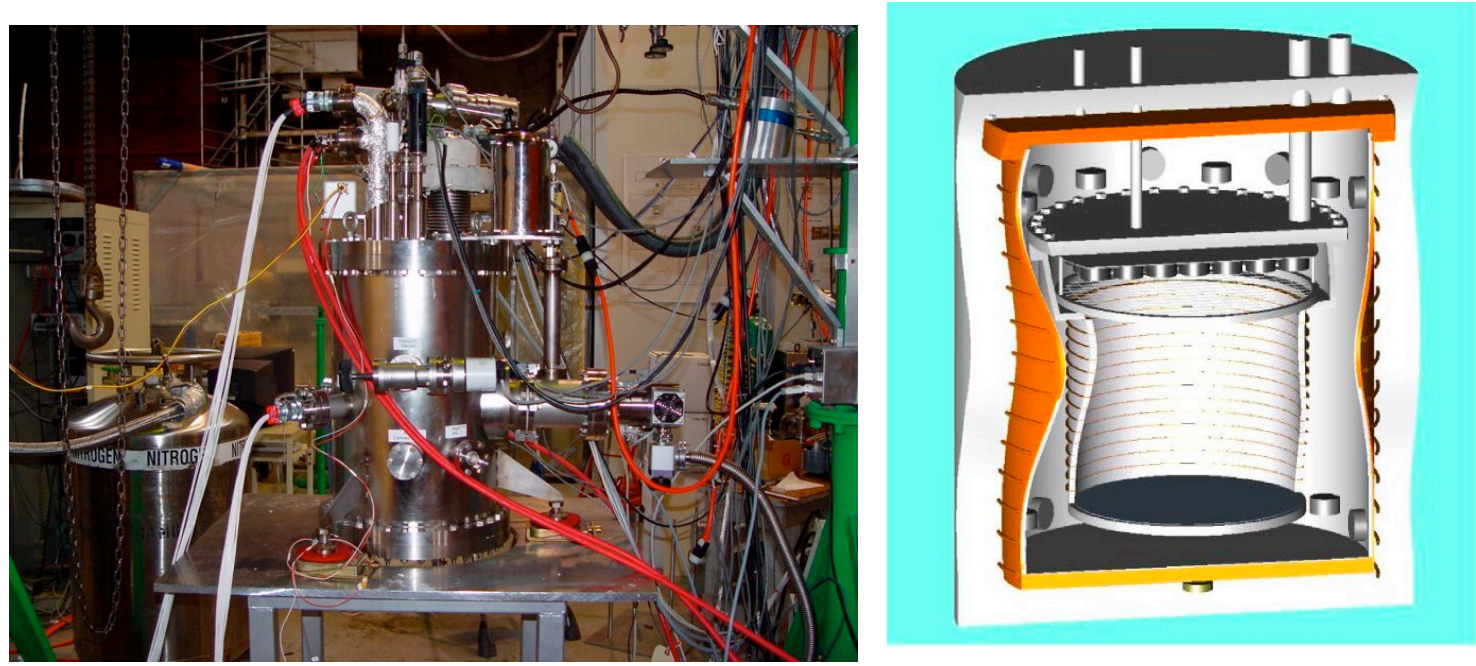

Figure 13: The left panel is a picture of the XENON detector during the first test at Columbia University in Januray 2006. A schematic view of the XENON setup is presented in the right panel. The detector is a time projection chamber filled with both liquid and gaseous xenon. Pictures from [42].

\section{XENON: Event Discrimination}

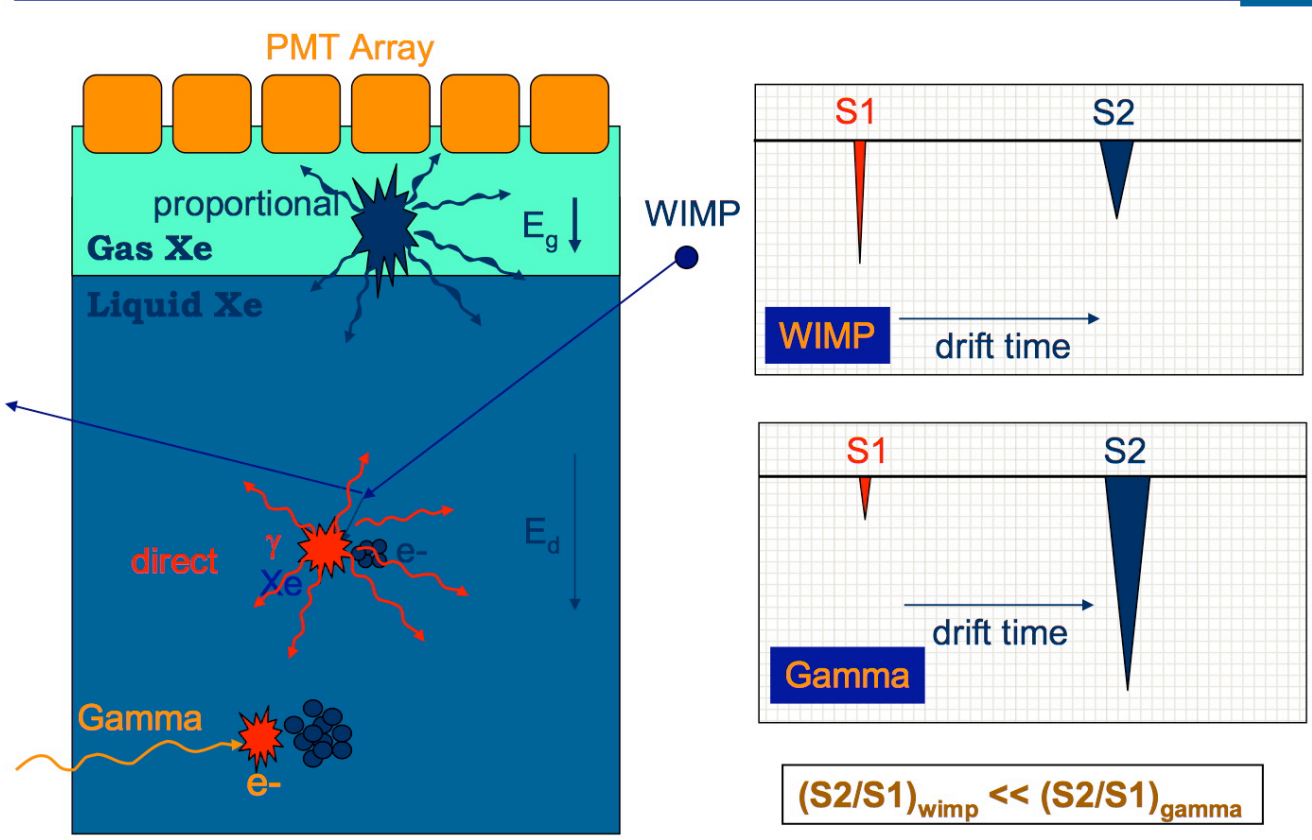

Figure 14: Events taking place inside liquid xenon generate a first pulse of light $S_{1}$ as well as some ionization. The resulting free electrons drift upward as they are dragged by the electric field established in the vessel. They move toward the anode located above the gaseous phase and are extracted from the liquid. As they accelerate in the gas gap, they collide upon xenon atoms and produce a second pulse of light $\mathrm{S}_{2}$. Diagram from [43]. 
inferred from the distribution of the PMT hits whereas the electron drift time allows to locate its depth inside the liquid phase. Electron recoils are characterized by a larger ionization than nuclear recoils. The measurement of the magnitude of the proportional scintillation $\mathrm{S}_{2}$ relative to the direct signal $S_{1}$ provides an efficient event-by-event discrimination down to a few $\mathrm{keV}$ nuclear recoil energy. The ionization yield parameter $y$ defined in the case of semiconductor experiments is now replaced by the proportional to direct signal ratio $\log _{10}\left(\mathrm{~S}_{2} / \mathrm{S}_{1}\right)$. This ionization estimator is plotted
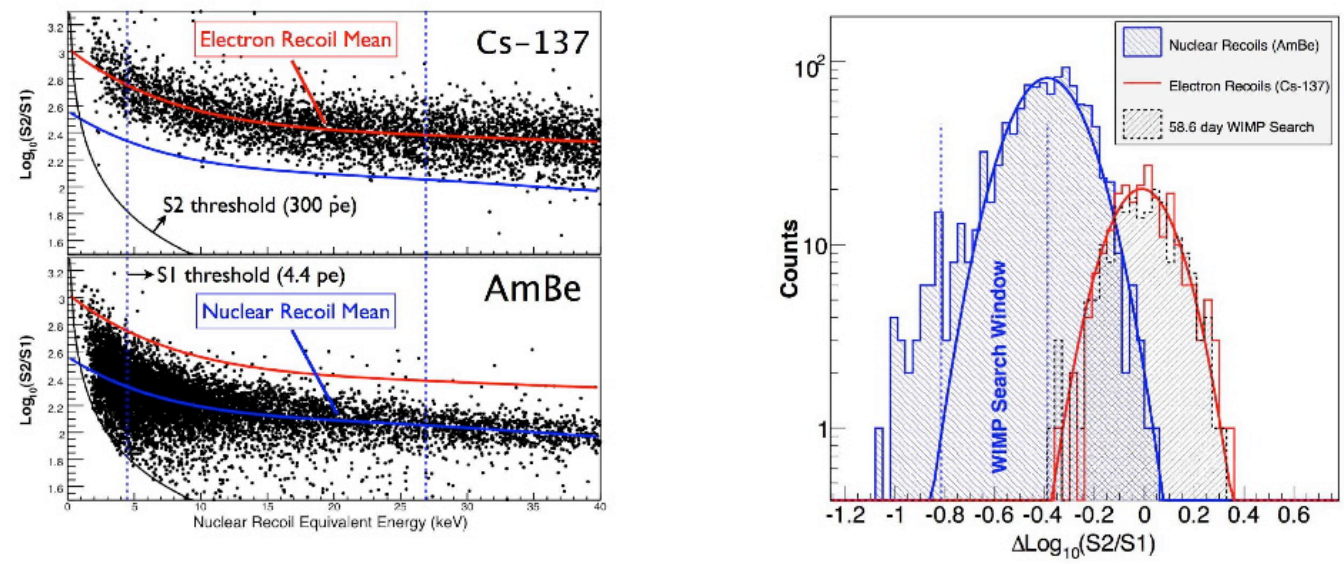

Figure 15: The ionization estimator $\log _{10}\left(\mathrm{~S}_{2} / \mathrm{S}_{1}\right)$ is plotted as a function of recoil energy for calibration data. A ${ }^{137} \mathrm{Cs}$ gamma ray source produces the population of electron recoils presented in the upper-left panel. The constellation of points in the lower-left panel correspond to the nuclear recoils generated by a AmBe neutron source. The right panel features the distribution of $\Delta \log _{10}\left(\mathrm{~S}_{2} / \mathrm{S}_{1}\right)$ for those electron (red) and nuclear (blue) events collected in the 6.7 to $9 \mathrm{keV}$ recoil energy bin. The results from the XENON WIMP search are also presented. Figures from [5].

as a function of recoil energy for the calibration data presented in figure 15. Two populations of events can be clearly separated. The electron recoils are generated by $\mathrm{a}^{137} \mathrm{Cs}$ gamma ray source. They are associated to high values of the $S_{2} / S_{1}$ ratio with an average given by the upper red curve. The nuclear recoils resulting from the impacts of the neutrons produced by a AmBe source have relatively weaker secondary scintillations. The lower blue curve indicates the mean value of the ionization estimator $\log _{10}\left(\mathrm{~S}_{2} / \mathrm{S}_{1}\right)$ for this band. The constellation of nuclear recoils lies almost entirely below the red curve. This enables the rejection of electron events. In the left panels, the vertical lines delineate the window selected for the WIMP search. In this energy range, electron and nuclear recoils can be efficiently discriminated. The calibration events of the right panel correspond to recoil energies from 6.7 to $9 \mathrm{keV}$. Two distributions for the $\Delta \log _{10}\left(\mathrm{~S}_{2} / \mathrm{S}_{1}\right)$ estimator are cleary visible. The electron events (red) are shifted by a factor of order 0.4 with respect to the nuclear recoils. The two vertical lines define the WIMP search window from which electron recoils are absent.

As discussed in section 1.1, the number of WIMP events $n_{\text {theo }}$ expected within the recoil energy acceptance window - from $E_{\mathrm{th}}$ to $E_{\max }$ - depends on the total WIMP-nucleus elastic scattering cross section (1.85). In the case of spin independent interactions, $\eta_{\text {theo }}$ is roughly given by relation (1.26) where an exposure of $1 \mathrm{~kg}$ day has been assumed. From an experimental point of view, the detector collects a total number $n_{\mathrm{obs}}$ of events in the signal window after various cuts have been 


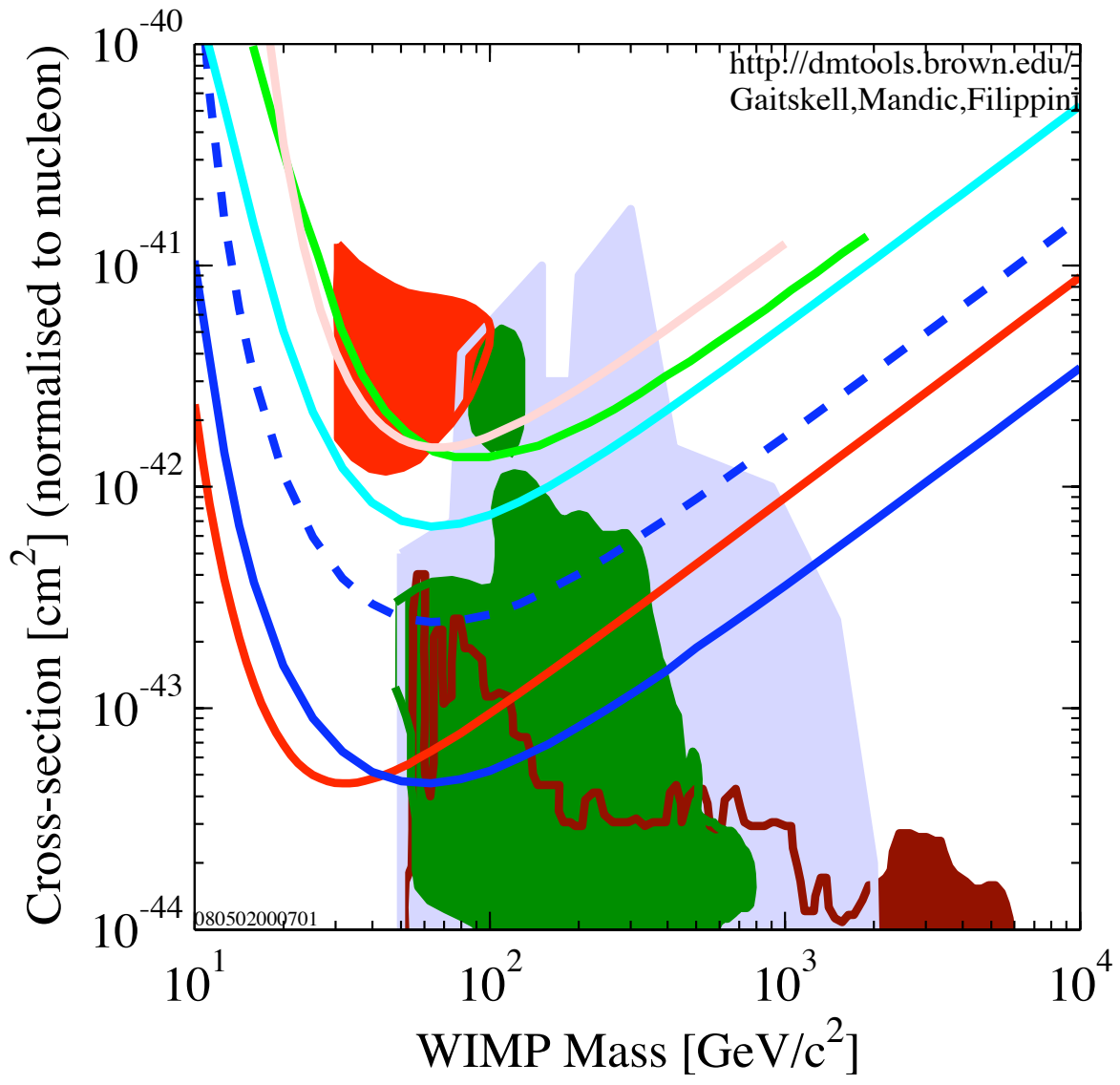

DATA listed top to bottom on plot

CRESST 200410.7 kg-day CaWO4

Edelweiss I final limit, 62 kg-days Ge 2000+2002+2003 limit

DAMA 2000 58k kg-days NaI Ann. Mod. 3sigma w/DAMA 1996

ZEPLIN II (Jan 2007) result

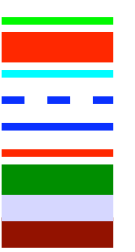

CDMS (Soudan) $2005 \mathrm{Ge}$ (10 keV threshold)

CDMS: $2004+2005$ (reanalysis) $+2008 \mathrm{Ge}$

XENON10 2007 (Net 136 kg-d)

Roszkowski/Ruiz de Austri/Trotta 2007, CMSSM Markov Chain Monte Carlos (

Baltz and Gondolo 2003

Baltz and Gondolo, 2004, Markov Chain Monte Carlos

080502000701

Figure 16: The experimental limits on the WIMP-nucleon spin independent elastic scattering cross section are featured as a function of WIMP mass in this typical exclusion diagram generated with the interactive plotter [44]. The colored regions delineate various theoretical predictions. They will soon be completely explored.

applied to reject the background. In practice, a few events are expected to leak from the electron recoil band and since they are misinterpreted as nuclear recoils, they contribute to $\eta_{\mathrm{bbs}}$. Neutronnucleus elastic collisions should also be taken into account hence an irreducible number $\beta_{\text {ack }}$ of background events. Large efforts are devoted by dedicated working groups [45] to simulate the neutron background and predict as accurately as possible $\eta_{\text {back }}$. This task is of paramount importance insofar as the number $n_{\text {WIMP }}$ of observed WIMP-nucleus interactions is eventually given by the difference between $n_{\text {obs }}$ and $n_{\text {back }}$. In the case of CDMS II, no event is reported in the search window [32] whereas XENON10 collects a statistics of $n_{\mathrm{obs}}=10$ nuclear recoils [5]. These are 
understood as statistical events leaking from the electron recoil band into the signal region or as multiple-scatter events with one scatter occuring below the cathode where it contributes only to $\mathrm{S}$ but not to $\mathrm{S}_{2}$, hence an experimental underestimation of the ionization.

Exercise $\mathbf{n}^{0}$ 1-l - Level [2] : A very large number $N$ of DM particles have crossed the detector at the end of an observation run. Each of these species has a vanishingly small probability $p$ to interact with a nucleus. In the limit where $N$ goes to infinity at constant $a=p \times N \equiv n_{\text {theo }}$, Poisson statistics applies. Show that the probability to detect actually $n$ WIMP-nucleus collisions is given by

$$
P\{n \mid a\}=\frac{a^{n}}{n !} e^{-a} .
$$

In the case of CDMS, no statistics is reported and the number of WIMP events effectively collected is $n_{\mathrm{WIMP}}=n_{\mathrm{obs}}=0$. Compute the theoretically expected number $n_{\text {theo }}$ above which the probability to have a null observation becomes less than $10 \%$. Justify then why the $90 \%$ confidence level limit on the WIMP-nucleus cross section is derived by merely setting $n_{\text {theo }}$ equal to 2.3 - see figure 4 .

The WIMP search by the CDMS and XENON collaborations has been so far negative. None of these experiments has claimed to have observed a single WIMP-nucleus scattering. In these conditions, a limit on the SI or SD WIMP-nucleon cross section can still be extracted as featured in figure 16. A naive derivation of such an exclusion plot is based on Poisson statistics. Once the cross section is specified, the number $n_{\text {theo }}$ of WIMP events theoretically expected at the end of a given run is fixed. The calculation takes into account in particular the WIMP mass and velocity distribution as well as the atomic number of the detecting medium and the experimental exposure. The probability that $n_{\mathrm{WIMP}}$ events are effectively collected is given by the Poisson distribution $P\left\{n_{\mathrm{WIMP}} \mid n_{\text {theo }}\right\}$ as defined in relation (1.90) where $n \equiv n_{\mathrm{WIMP}}$ and $a \equiv n_{\text {theo }}$. A limit is obtained on the WIMP-nucleon cross section with a confidence level of C.L. by excluding configurations for which

$$
P\left\{n_{\text {WIMP }} \mid n_{\text {theo }}\right\} \leq(1-\text { C.L. }) \text {. }
$$

In the case of CDMS II for instance, no WIMP event is reported. The probability for such a null observation amounts to $10 \%$ when the theoretical number $n_{\text {theo }}$ is equal to $\ln (10) \sim 2.3$. This value corresponds actually to a Poisson probability of $P\{0 \mid 2.3\}=0.1$ to get no event given that 2.3 are theoretically expected. Cross sections for which $n_{\text {theo }}$ is even larger are therefore excluded at the $90 \%$ confidence level.

Because the possibility of an additional and unknown source of background cannot be avoided, the substraction between $n_{\mathrm{obs}}$ and $n_{\text {back }}$ is not performed since the latter is still subject to some uncertainty. That is why XENON10 gets for instance a conservative limit on the WIMP-nucleon cross section by replacing $n_{\mathrm{WIMP}}-$ which is consistent with 0 - by its collected statistics of $n_{\mathrm{obs}}=10$ nuclear recoils. The method outlined above is therefore very sensitive to $\eta_{\mathrm{bbs}}$ which depends itself on the recoil energy interval. This window can be inadvertently selected so as to minimize the number of collected events, hence a stronger constraint on the cross section than justified by the data. The maximum gap method and the optimum interval technique have been devised to remedy this problem as explained in [46]. 
Several improvements are finally foreseen for the future. Recoils resulting from neutrons boucing off the nuclei of a ton size detector can be identified by the presence of several elastic scatterings associated to the same event. The corresponding cross section is in the barn range. A single neutron undergoes several collisions in a large instrument and leaves a multiple-scatter imprint. The rejection of neutron induced nuclear recoils should be improved by increasing the detecting mass and also by segmenting the instrument. Multiple nuclear recoils taking place at the same time in various portions of the detector can be rejected as neutron events. The annual modulation of the WIMP signal has already been discussed in section 1.1 and provides a tool to select the interesting events. The magnitude of this effect is small though. More exciting is the diurnal modulation of the average direction of the nuclear recoils associated to WIMP scatterings. The arrival direction of the impinging DM particles varies substantially in the laboratory between daytime and night as a result of the Earth rotation. If the directions of the recoils are known, a dozen of events have been shown to be suffficient to disentangle a WIMP signal from an isotropic background [47].

WIMP direct detection is actually a fast moving field with impressive advances achieved in the past 20 years. The expected signal from DM particles impinging on a terrestrial instrument is mildly subject to astrophysical uncertainties though variations by a factor of ten - arising from the local DM density $\rho_{\odot}$ and the WIMP velocity distribution - are not unreasonable. More troublesome are the theoretical uncertainties related to the quark spin and mass content of the nucleon as well as to nuclear matrix elements. Experimentally, the zeptobarn goal is reacheable in the near future with ton size detectors. The top of the SUSY colored regions of figure 16 has already been explored. Note finally the existence of some complementarity between LHC and direct detection. The former is sensitive to WIMP masses below a few hundreds of $\mathrm{GeV}$ while the latter can probe much larger masses provided that the WIMP-nucleon scattering cross section is larger than $\sim 1 \mathrm{zb}$. 


\section{The messengers of DM annihilation}

Should DM species pervade the halo of the Milky Way, their mutual annihilations would yield several indirect signatures. These are potentially detectable on Earth under the form of spectral distortions appearing in various cosmic radiations

$$
\chi+\chi \rightarrow q \bar{q}, W^{+} W^{-}, \ldots \rightarrow \bar{p}, \bar{D}, e^{+} \gamma \& v^{\prime} s .
$$

Detection of the DM annihilation products has motivated the spectacular development of several new experimental techniques. Searches for antiprotons and positrons are performed by balloon and satellite-borne devices. Because the flux depends on the square of the WIMP density $n_{\chi}$, the limit which may be set on the annihilation cross section scales approximately as $m_{\chi}{ }^{2}$. That type of search is mostly sensitive to small WIMP masses but no distortion has been reported at low energy. The $\mathrm{TeV}$ window has become therefore the new frontier. High-energy photons are detected both by air Cerenkov telescopes (ACT) and by satellite-borne instruments. The WIMP annihilation rate and hence the gamma ray signal both scale as $m_{\chi}{ }^{-2}$. Because of the background in which that signal is swamped, the experimental reach on the annihilation cross section approximately scales as the mass $m_{\chi}$. This behaviour is reminiscent of the exclusion plot presented in figure 4 where the upper bound on the elastic scattering cross section $\sigma_{p}^{\text {SI }}$ increases linearly with $m_{\chi}$ in the large mass regime. The neutrino channel is most sensitive to large values of $m_{\chi}$. The limit which may be set on the annihilation cross section does not depend too much on the WIMP mass and this channel is complementary to the other searches.

Various species can be produced by WIMP annihilations among which are antimatter cosmic rays, high-energy photons and neutrinos. The corresponding rate $q(\mathbf{x}, E)$ for the production of these particles depends on their energy $E$ and is related to the WIMP annihilation cross section $\sigma_{\text {ann }}$ through

$$
q(\mathbf{x}, E)=\eta\left\langle\sigma_{\mathrm{ann}} v\right\rangle\left\{\frac{\rho(\mathbf{x})}{m_{\chi}}\right\}^{2} f(E) .
$$

The coefficient $\eta$ is a quantum factor equal to $1 / 2$ for a self-conjugate particle like a Majorana fermion or to $1 / 4$ otherwise. The annihilation cross section is averaged over the momenta of the incoming DM particles to yield $\left\langle\sigma_{\mathrm{ann}} v\right\rangle$ whose value depends on the specific microscopic interactions at stake. Finally, the energy distribution $d N / d E$ of the species produced in a single annihilation event is generically denoted by $f(E)$.

\subsection{Cosmic ray transport : a short overview}

Whatever the mechanism responsible for their production, charged cosmic rays subsequently propagate through the galactic magnetic field and are deflected by its irregularities : the Alfvén waves. In the regime where the magnetic turbulence is strong - which is the case for the Milky Way - cosmic ray transport needs to be investigated numerically. Monte Carlo simulations [48] indicate that it is similar to space diffusion with a coefficient

$$
K(E)=K_{0} \beta(\mathscr{R} / 1 \mathrm{GV})^{\delta},
$$

which increases as a power law with the rigidity $\mathscr{R}=p / q$ of the particle. In addition, because the scattering centers drift inside the Milky Way with a velocity $V_{a} \sim 20$ to $100 \mathrm{~km} \mathrm{~s}^{-1}$, a second 
order Fermi mechanism is responsible for some mild diffusive reacceleration. Its coefficient $K_{E E}$ depends on the particle velocity $\beta$ and total energy $E$ and is related to the space diffusion coefficient $K(E)$ through

$$
K_{E E}=\frac{2}{9} V_{a}^{2} \frac{E^{2} \beta^{4}}{K(E)}
$$

In the case of positrons, diffusive reacceleration is completely dominated by energy losses. Finally, galactic convection wipes cosmic rays away from the disc with a velocity $V_{C} \sim 5$ to $15 \mathrm{~km} \mathrm{~s}^{-1}$.

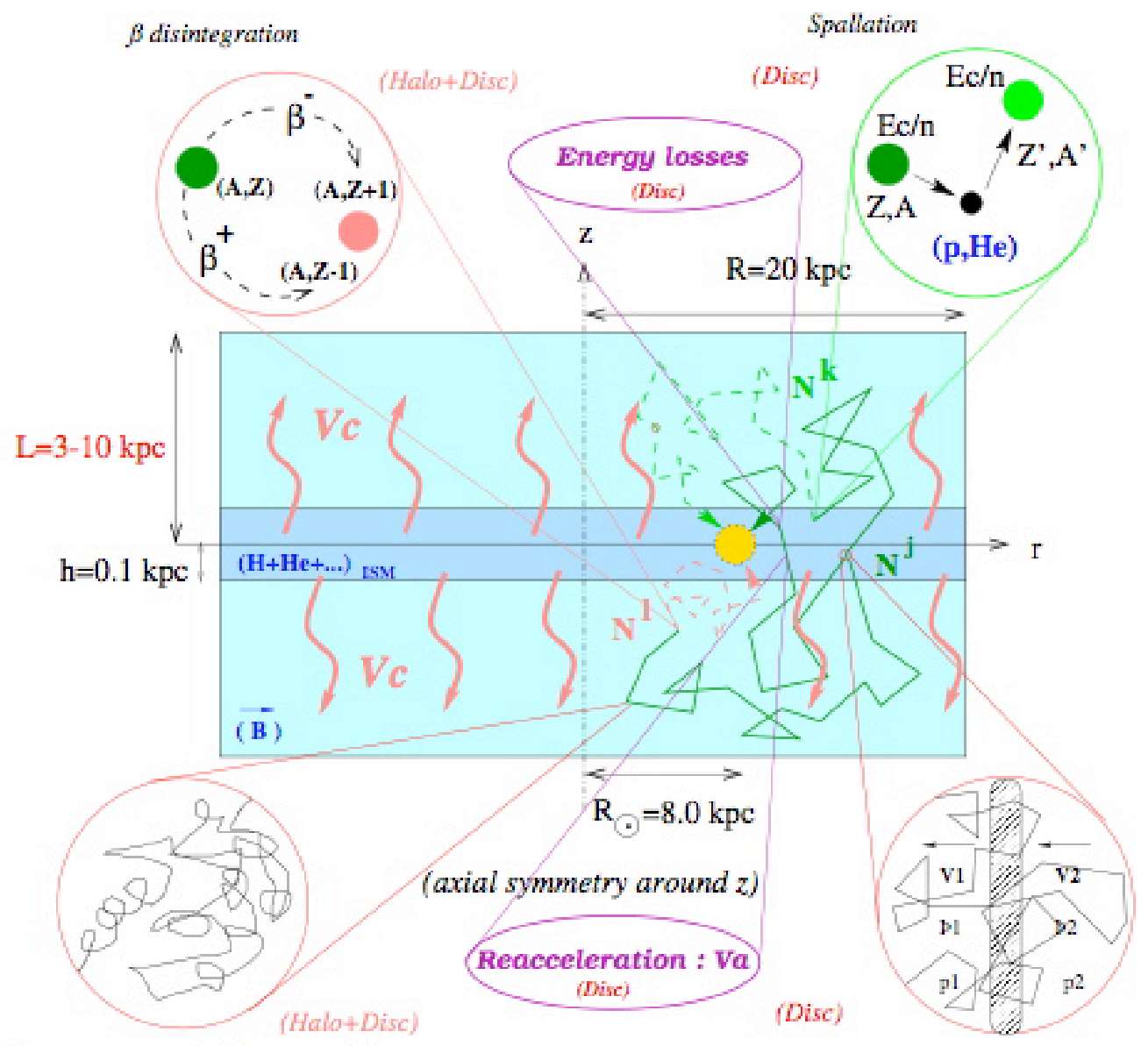

Diffusion on magnetic inhomogeneities

$\boldsymbol{R}^{0.6}$

Acceleration by shock waves

$\boldsymbol{R}^{-2.2}$

Figure 17: Schematic edge-on view of the Milky Way diffusive halo $(\mathrm{DH})$ as seen by a cosmic ray physicist. The stellar and gaseous disc is sandwiched between two thick layers which contain turbulent magnetic fields. After having been accelerated by SN driven shock waves or produced by DM species annihilating in the galactic halo, cosmic rays diffuse on magnetic inhomogeneities and are wiped away by a galactic wind with velocity $V_{C}$. They can lose energy and are also mildly subject to diffusive reacceleration. The former process is by far the dominant one in the case of electrons and positrons. This diagram has been borrowed from the review [49]. 
After this short digest of cosmic ray transport, we can assume a steady state for the various populations of particles and write the master equation fulfilled by the space and energy distribution function $\psi=d n / d E$ as

$$
\partial_{z}\left(V_{C} \psi\right)-K \Delta \psi+\partial_{E}\left\{b^{\operatorname{loss}}(E) \psi-K_{E E}(E) \partial_{E} \psi\right\}=q(\mathbf{x}, E)
$$

This equation applies to any species - protons, antiprotons or positrons - as long as the rates for production $q$ and energy loss $b^{\text {loss }}(E)$ are properly accounted for. It has been solved within the framework of the semi-analytical two-zone model which has been extensively discussed in previous works $[50,51]$ and whose salient features we briefly recall now. According to our approach, steady state is assumed and the region of the Galaxy inside which cosmic rays diffuse - the socalled diffusive halo or $\mathrm{DH}$ - is pictured as a thick disc which matches the circular structure of the Milk Way as shown in figure 17. The galactic disc of stars and gas, where primary cosmic rays are accelerated, lies in the middle. It extends radially $20 \mathrm{kpc}$ from the center and has a half-thickness $h$ of $100 \mathrm{pc}$. Confinement layers where cosmic rays are trapped by diffusion lie above and beneath this thin disc of gas. The intergalactic medium starts at the vertical boundaries $z= \pm L$ as well as beyond a radius of $r=R \equiv 20 \mathrm{kpc}$. Notice that the half-thickness $L$ of the diffusive halo is not known and reasonable values range from 1 to $15 \mathrm{kpc}$. The diffusion coefficient $K$ is the same everywhere whereas the convective velocity is exclusively vertical with component $V_{C}(z)=V_{C} \operatorname{sign}(z)$. This galactic wind, which is produced by the bulk of the disc stars like the Sun, drifts away from its progenitors along the vertical directions, hence the particular form assumed here for $V_{C}$. Notice also that the normalization coefficient $K_{0}$, the index $\delta$, the galactic drift velocity $V_{C}$ and the Alfvén velocity $V_{a}$ are all unknown. This situation may be remedied with the help of the boron to carbon $\mathrm{B} / \mathrm{C}$ ratio which is quite sensitive to cosmic ray transport and which may be used as a constraint. The three propagation models featured in table 4 have been drawn from [52]. The MED configuration provides the best fit to the $\mathrm{B} / \mathrm{C}$ measurements whereas the MIN and MAX models lead respectively to the minimal and maximal allowed antiproton fluxes which can be produced by WIMP annihilation.

\begin{tabular}{|c||c|c|c|c|c|}
\hline Case & $\delta$ & $K_{0}\left[\mathrm{kpc}^{2} / \mathrm{Myr}\right]$ & $L[\mathrm{kpc}]$ & $V_{C}[\mathrm{~km} / \mathrm{s}]$ & $V_{a}[\mathrm{~km} / \mathrm{s}]$ \\
\hline \hline MIN & 0.85 & 0.0016 & 1 & 13.5 & 22.4 \\
MED & 0.70 & 0.0112 & 4 & 12 & 52.9 \\
MAX & 0.46 & 0.0765 & 15 & 5 & 117.6 \\
\hline
\end{tabular}

Table 4: Typical combinations of diffusion parameters that are compatible with the $\mathrm{B} / \mathrm{C}$ analysis [50]. As shown in [52], these propagation models correspond respectively to minimal, medium and maximal primary antiproton fluxes.

The solution of the master equation (2.5) may be generically expressed as the integral

$$
\psi(\odot, E)=\int d E_{S} \int_{\mathrm{DH}} d^{3} \mathbf{x}_{S} G\left(\mathbf{x}_{\odot}, E \leftarrow \mathbf{x}_{S}, E_{S}\right) q\left(\mathbf{x}_{S}, E_{S}\right) .
$$

The energy $E_{S}$ at the source runs over a range which depends on the nature of the cosmic ray species as discussed below. The space integral is performed over the diffusive halo. The convolution (2.6) involves the Green function $G$ which describes the probability for a cosmic ray that is produced at 
location $\mathbf{x}_{S}$ with the energy $E_{S}$ to reach the Earth where it is detected with the degraded energy $E$. The cosmic ray space and energy density $\psi$ can be translated into the differential flux

$$
\Phi=\frac{\beta}{4 \pi} \psi
$$

where $\beta$ stands for the particle velocity. This flux is expressed in units of $\mathrm{m}^{-2} \mathrm{~s}^{-1} \mathrm{sr}^{-1} \mathrm{GeV}^{-1}$.

Exercise $\mathbf{n}^{0}$ 2-a - Level [1]: Show that the flux of antiprotons or positrons produced by WIMP annihilations may be written as the product

$$
\Phi(\odot, E)=\mathscr{F} \int d E_{S} f\left(E_{S}\right) I\left(E, E_{S}\right),
$$

where the information related to particle physics has been factored out in

$$
\mathscr{F}=\eta \frac{\beta}{4 \pi}\left\langle\sigma_{\mathrm{ann}} v\right\rangle\left\{\frac{\rho_{\odot}}{m_{\chi}}\right\}^{2} .
$$

The energy distribution $f\left(E_{S}\right)$ describes the spectrum at the source and depends on the details of the WIMP annihilation mechanism.

The information on the galactic DM density profile $\rho$ as well as on the propagation of cosmic rays within the Milky Way DH is summarized in the halo integral

$$
I\left(E, E_{S}\right)=\int_{\mathrm{DH}} d^{3} \mathbf{x}_{S} G\left(\mathbf{x}_{\odot}, E \leftarrow \mathbf{x}_{S}, E_{S}\right)\left\{\frac{\rho\left(\mathbf{x}_{S}\right)}{\rho_{\odot}}\right\}^{2},
$$

where the solar neighborhood DM density is denoted by $\rho_{\odot}$. The halo integral $I\left(E, E_{S}\right)$ is a key ingredient for the derivation of the flux at the Earth of the antimatter species produced inside the galactic DH by WIMP annihilations. The spatial reach of the Green function $G$ depends on the nature of the cosmic ray particles - either antiprotons or positrons - and on the energies $E$ and $E_{s}$. This range delineates the region of the Milky Way from which most of the signal detected at the Earth originates. It corresponds to the extension of the so-called horizon beyond which the Green function vanishes. The horizon plays a crucial role in the subsequent discussion as well as in the next section 3 devoted to the effect of DM clumps.

\subsection{TeV antiprotons : a new window}

The propagation of cosmic ray antiprotons is dominated by diffusion. Energy losses as well as diffusive reacceleration do not play any major role. A very crude approximation for the antiproton Green function is obtained by neglecting galactic convection and solving the resulting Poisson equation in infinite space. This yields the antiproton propagator

$$
G_{\overline{\mathrm{p}}}\left(\mathbf{x}_{\odot} \leftarrow \mathbf{x}_{S}\right) \equiv \frac{1}{4 \pi K(E)} \frac{1}{r_{\oplus}}
$$

where $r_{\oplus}$ denotes the distance between the Earth and the source. The sole merit of this expression is to exhibit the importance of remote sources. We will therefore keep in mind that the antiproton 
sphere is fairly extended. The finite thickness of the diffusive halo is nevertheless a limiting factor since cosmic rays may escape through the vertical boundaries as they wander toward the Earth. As a consequence, the size of the antiproton sphere cannot be much larger than the DH halfthickness $L$. Galactic convection comes also into play. If the wind velocity $V_{C}$ is large, cosmic rays are efficiently blown outside the Milky Way. This process limits further the reach of the antiproton sphere. The solution of the master equation (2.5) has been thoroughly investigated and several different techniques $[53,54]$ lead essentially to the same fluxes at the Earth.

The Bessel expansion method takes advantage of the axial symmetry of the DH and enforces a vanishing cosmic ray flux at a distance $R=20 \mathrm{kpc}$ from the rotation axis of the Galaxy. This condition is actually implemented naturally by the following series expansion for $\psi$

$$
\psi(r, z, E)=\sum_{i=1}^{+\infty} P_{i}(z, E) J_{0}\left(\alpha_{i} r / R\right)
$$

The Bessel function of zeroth order $J_{0}$ vanishes at the points $\alpha_{i}$. The radial dependence of $\psi$ is now taken into account by the set of its Bessel transforms $P_{i}(z, E)$. The source term $q$ may also be Bessel expanded into the corresponding functions $Q_{i}(z, E)$ so that the master equation (2.5) becomes

$$
\begin{aligned}
\partial_{z}\left(V_{C} P_{i}\right) & -K \partial_{z}^{2} P_{i}+K\left\{\frac{\alpha_{i}}{R}\right\}^{2} P_{i}+ \\
& +2 h \delta(z) \partial_{E}\left\{b^{\operatorname{loss}}(E) P_{i}-K_{E E}(E) \partial_{E} P_{i}\right\}=Q_{i}(z, E) .
\end{aligned}
$$

Here, energy loss and diffusive reacceleration are confined inside the galactic disc - which is considered infinitely thin, hence the presence of an effective term $2 h \delta(z)$. The form of the source terms $Q_{i}(z, E)$ which appear in equation (2.13) depends on the nature of the cosmic ray particle. In the case of antiprotons, the following mechanisms can in principle contribute.

(i) Antiprotons may collide elastically on interstellar $\mathrm{H}$ and He. Because they are preferentially scattered forward, however, such interactions are innocuous and will be disregarded.

(ii) Antiprotons may also annihilate on interstellar $\mathrm{H}$ and $\mathrm{He}$. This leads to a negative source term $-\Gamma_{\overline{\mathrm{p}}}^{\mathrm{ann}} \psi$, where the annihilation rate $\Gamma_{\overline{\mathrm{p}}}^{\mathrm{ann}}$ is defined as

$$
\Gamma_{\overline{\mathrm{p}}}^{\mathrm{ann}}=\sigma_{\overline{\mathrm{p}} \mathrm{H}}^{\mathrm{ann}} \beta_{\overline{\mathrm{p}}} n_{\mathrm{H}}+\sigma_{\overline{\mathrm{p}} \mathrm{He}}^{\mathrm{ann}} \beta_{\overline{\mathrm{p}}} n_{\mathrm{He}} .
$$

The annihilation cross section $\sigma_{\overline{\mathrm{p}} \mathrm{H}}^{\mathrm{ann}}$ can be borrowed from $[55,56]$ and multiplied by a factor of $4^{2 / 3} \sim 2.5$, taking into account the higher geometric cross section, to get $\alpha_{\mathrm{p} H e}^{\mathrm{ann}}$. The average hydrogen $n_{\mathrm{H}}$ and helium $n_{\mathrm{He}}$ densities in the galactic disc are respectively set equal to 0.9 and 0.1 $\mathrm{cm}^{-3}$.

(iii) The annihilation of DM candidate particles throughout the Milky Way halo generates primary antiprotons. The corresponding source term $q_{\bar{p}}^{\text {prim }}(r, z, E)$ has already been discussed and is generically given by expression (2.2) where $f(E)$ stands here for the antiproton spectrum $d N_{\overline{\mathrm{p}}} / d E_{\overline{\mathrm{p}}}$. Notice that WIMP annihilations take place all over the diffusive halo.

(iv) Such is not the case with either secondary antiprotons - which are produced as high-energy primary nuclei impinge on the atoms of the interstellar medium inside the galactic disc - or tertiary antiprotons which result from the inelastic and non-annihilating interactions which these particles 
may undergo with the same atoms. The rate for the production of secondary antiprotons takes the following form

$$
q_{\overline{\mathrm{p}}}^{\mathrm{sec}}\left(r, E_{\overline{\mathrm{p}}}\right)=\int_{E_{\mathrm{p}}^{0}}^{+\infty} n_{\mathrm{H}} \times \beta_{\mathrm{p}} \psi_{\mathrm{p}}\left(r, E_{\mathrm{p}}\right) \times d E_{\mathrm{p}} \times \frac{d \sigma}{d E_{\overline{\mathrm{p}}}}\left(E_{\mathrm{p}} \rightarrow E_{\overline{\mathrm{p}}}\right)
$$

in the case of the interactions between cosmic ray protons and hydrogen atoms. In figure 18, the various contributions to the secondary antiproton flux from the spallation of interstellar $\mathrm{H}$ and $\mathrm{He}$ by cosmic ray protons and alpha particles are presented together with the existing low-energy data. Galactic propagation parameters correspond to the MED configuration of table 4.

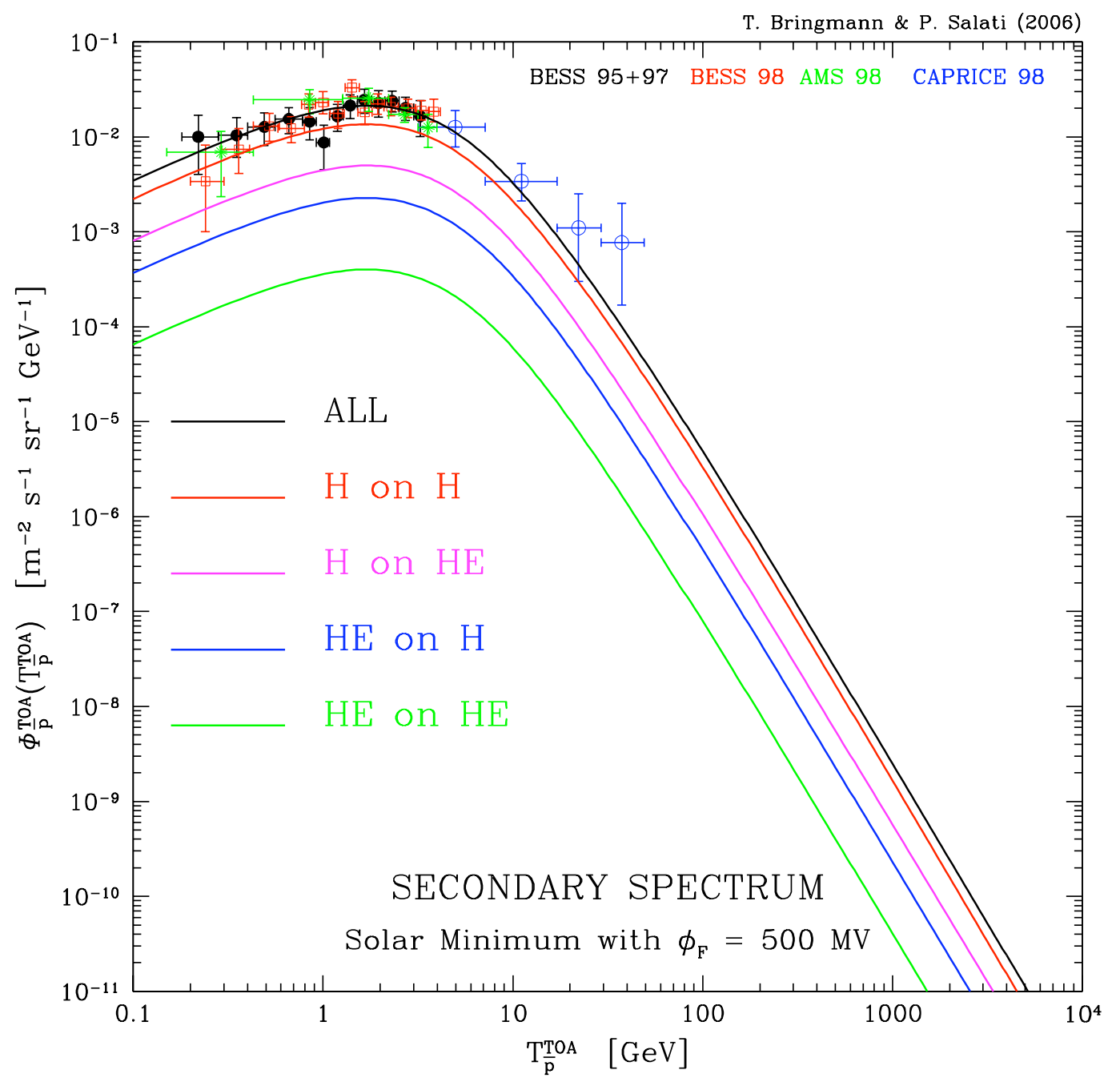

Figure 18: The spallation of the interstellar medium by cosmic ray nuclei yields a flux of secondary antiprotons which is plotted here as a function of their kinetic energy as measured at the top of the atmosphere (TOA). Propagation parameters correspond to the MED configuration of table 4. For reference, the existing low-energy data on the antiproton flux at the top of the atmosphere $[57,58,59,60]$ are also presented. Solar modulation has been implemented through the force field approximation [61], with a Fisk potential $\phi_{\mathrm{F}}$ of $500 \mathrm{MV}$ corresponding to the minimum of solar activity during which the observations have been performed. Figure from [53]. 
(v) Antiprotons may finally collide on a nucleon at rest and transfer enough energy to excite it as a $\Delta$ resonance. This mechanism redistributes antiprotons toward lower energies and flattens their spectrum as shown in [62]. This yields the source term

$$
\begin{aligned}
q_{\overline{\mathrm{p}}}^{\text {ter }}\left(r, E_{\overline{\mathrm{p}}}\right) & =\int_{E_{\overline{\mathrm{p}}}}^{+\infty} \frac{d \sigma_{\overline{\mathrm{p}} \mathrm{H} \rightarrow \overline{\mathrm{p} X}}}{d E_{\overline{\mathrm{p}}}}\left(E_{\overline{\mathrm{p}}}^{\prime} \rightarrow E_{\overline{\mathrm{p}}}\right) n_{\mathrm{H}} \beta_{\overline{\mathrm{p}}}^{\prime} \psi_{\overline{\mathrm{p}}}\left(r, E_{\overline{\mathrm{p}}}^{\prime}\right) d E_{\overline{\mathrm{p}}}^{\prime} \\
& -\sigma_{\overline{\mathrm{p}} \mathrm{H} \rightarrow \overline{\mathrm{p}} \mathrm{X}}\left(E_{\overline{\mathrm{p}}}\right) n_{\mathrm{H}} \beta_{\overline{\mathrm{p}}} \psi_{\overline{\mathrm{p}}}\left(r, E_{\overline{\mathrm{p}}}\right)
\end{aligned}
$$

where the inelastic and non-annihilating differential cross section in this expression can be approximated by

$$
\frac{d \sigma_{\overline{\mathrm{p}} \mathrm{H} \rightarrow \overline{\mathrm{p}} \mathrm{X}}}{d E_{\overline{\mathrm{p}}}}=\frac{\sigma_{\overline{\mathrm{p}} \mathrm{H} \rightarrow \overline{\mathrm{p}} \mathrm{X}}}{T_{\overline{\mathrm{p}}}^{\prime}} .
$$

The initial antiproton kinetic energy is denoted by $T_{\overline{\mathrm{p}}}^{\prime}$. In order to take into account elastic scatterings on helium, one simply has to replace the hydrogen density by $n_{\mathrm{H}}+4^{2 / 3} n_{\mathrm{He}}$.

Exercise $\mathbf{n}^{0} \mathbf{2}-\mathbf{b}$ - Level [2] : Equipped with all these source terms, derive the full expression for the master equation describing the (Bessel transformed) antiproton distribution functions $\bar{P}_{i}(z, E)$ and show that

$$
\begin{aligned}
\partial_{z}\left(V_{C} \bar{P}_{i}\right) & -K \partial_{z}^{2} \bar{P}_{i}+K\left\{\frac{\alpha_{i}}{R}\right\}^{2} \bar{P}_{i}+2 h \delta(z) \partial_{E}\left\{b^{\text {loss }}(E) \bar{P}_{i}-K_{E E}(E) \partial_{E} \bar{P}_{i}\right\}= \\
& -2 h \delta(z) \Gamma_{\overline{\mathrm{p}}}^{\text {ann }} \bar{P}_{i}+Q_{\overline{\mathrm{p}}, i}^{\text {prim }}(z, E)+2 h \delta(z)\left\{Q_{\overline{\mathrm{p}}, i}^{\mathrm{sec}}+Q_{\overline{\mathrm{p}}, i}^{\mathrm{ter}}\right\} .
\end{aligned}
$$

Integrate this relation along the vertical axis $z$ - in particular through the infinitely thin disc - in order to establish eventually that the Bessel transforms $\bar{P}_{i}(0, E)$ fulfill the integro-differential equation

$$
\begin{aligned}
\overline{\mathscr{A}}_{i} \bar{P}_{i}(0, E)+ & 2 h \partial_{E}\left\{b^{\operatorname{loss}}(E) \bar{P}_{i}(0, E)-K_{E E}(E) \partial_{E} \bar{P}_{i}(0, E)\right\}= \\
& 2 h\left\{Q_{\overline{\mathrm{p}}, i}^{\mathrm{sec}}+Q_{\overline{\mathrm{p}}, i}^{\mathrm{ter}}\right\}+2 \int_{0}^{L} d z Q_{\overline{\mathrm{p}}, i}^{\mathrm{prim}}(z, E) e^{-\frac{z V_{C}}{2 K} \mathscr{F}_{i}(z) .}
\end{aligned}
$$

Check that the coefficients $\overline{\mathscr{A}}_{i}$ are given by

$$
\overline{\mathscr{A}}_{i}(E)=V_{C}+2 h \Gamma_{\overline{\mathrm{p}}}^{\mathrm{ann}}(E)+K(E) S_{i} \operatorname{coth}\left(\frac{S_{i} L}{2}\right),
$$

where $S_{i}^{2} \equiv\left(V_{C} / K\right)^{2}+\left(2 \alpha_{i} / R\right)^{2}$ while the vertical functions $\mathscr{F}_{i}(z)$ are defined by

$$
\mathscr{F}_{i}(z)=\sinh \left\{\frac{S_{i}}{2}(L-z)\right\} / \sinh \left\{\frac{S_{i}}{2} L\right\} .
$$

Equation (2.19) may be solved according to the method outlined in the appendix B of [51]. Setting the energy loss rate $b^{\text {loss }}$ and the energy diffusion coefficient $K_{E E}$ equal to zero does not affect too much the result.

A completely different approach of the antiproton transport through the DH relies on the existence of the above mentioned Green propagator $G_{\overline{\mathrm{p}}}$. Such a function translates the probability for 
an antiproton produced at point $S\left(x_{S}, y_{S}, z_{S}\right)$ to travel to the observer located at point $M(x, y, z)$. Energy losses, diffusive reacceleration and tertiary production are inefficient above a few $\mathrm{GeV}$ and can be safely neglected. The energies $E$ and $E_{S}$ are thus identical and the antiproton energy spectrum at the Earth is given by the simplified convolution

$$
\psi_{\overline{\mathrm{p}}}(\odot, E)=\int_{\mathrm{DH}} d^{3} \mathbf{x}_{S} G_{\overline{\mathrm{p}}}\left(\mathbf{x}_{\odot} \leftarrow \mathbf{x}_{S}, E\right) q_{\overline{\mathrm{p}}}\left(\mathbf{x}_{S}, E\right) .
$$

The construction of the Green function for antiprotons is inspired from the positron case - see section 2.3 - with the essential difference that the antiproton energy does not change and that time is integrated out.

Exercise $\mathbf{n}^{0}$ 2-c - Level [3] : Because the Milky Way is now pictured as an infinite slab of halfthickness $L$ with a gaseous disc in the middle at $z=0$, the antiproton propagation is invariant under a translation along the horizontal directions $x$ and $y$. The master equation (2.5) needs still to be solved along the vertical direction $z$ with the condition that $G_{\bar{p}}$ vanishes at the boundaries $z= \pm L$. Energy losses, diffusive reacceleration and tertiary production are neglected. Taking only into account space diffusion, galactic convection and antiproton annihilations on interstellar $\mathrm{H}$ and $\mathrm{He}$, establish that

$$
G_{\overline{\mathrm{p}}}\left(\mathbf{x}_{\odot} \leftarrow \mathbf{x}_{S}, E\right)=\frac{e^{-z_{S} / r_{w}}}{2 \pi K(E)} \sum_{n=1}^{+\infty} \frac{1}{C_{n}} \phi_{n}(0) \phi_{n}\left(z_{S}\right) K_{0}\left(\frac{r}{L} \sqrt{\varepsilon_{n}}\right) .
$$

The vertical functions $\phi_{n}$ are defined by

$$
\phi_{n}(z)=\sin \left\{\xi_{n}\left(1-\frac{z}{L}\right)\right\},
$$

where the coefficients $\xi_{n}$ are solutions to the equation

$$
\xi_{n}=n \pi-\tan ^{-1}\left(p \xi_{n}\right) .
$$

Check that the parameter $p$ is related to the convective scale $r_{w} \equiv 2 K(E) / V_{C}$ and the scattering length $r_{s} \equiv K(E) / h \Gamma_{\overline{\mathrm{p}}}^{\mathrm{ann}}(E)$ through the identity

$$
\frac{1}{p}=\frac{L}{r_{s}}+\frac{L}{r_{w}},
$$

whereas the scale $C_{n}$ is defined by

$$
\frac{C_{n}}{L}=1+\frac{1}{p}\left(\frac{\sin \xi_{n}}{\xi_{n}}\right)^{2}
$$

In the argument of the modified Bessel functions of the second kind $K_{0}$ in equation (2.23), the ratio $r / L$ is multiplied by a factor $\sqrt{\varepsilon_{n}}$ where

$$
\varepsilon_{n}=\xi_{n}^{2}+\left(\frac{L}{r_{w}}\right)^{2}
$$

At high energies, above $\sim 100 \mathrm{GeV}, r_{s}$ and $r_{w}$ are much larger than $L$ due to the greatly enhanced diffusion coefficient $K(E)$. As a consequence, the parameter $p$ is much larger than 1 and thus the 
coefficient $\xi_{n}$ is approximately given by

$$
\xi_{n}=(n-1 / 2) \pi
$$

Furthermore, the scale $C_{n}$ tends to $L$ while $\sqrt{\varepsilon_{n}}$ reduces to $\xi_{n}$. When diffusion takes over disc annihilations and galactic convection, the antiproton propagator $G_{\bar{p}}$ depends on the energy $E$ only through the factor

$$
G_{\overline{\mathrm{p}}} \propto \frac{e^{-z_{S} / r_{w}}}{2 \pi K(E)} \simeq \frac{1}{2 \pi K(E)} .
$$

The ratio $z_{S} / r_{w}$ is actually bounded by $L / r_{w} \ll 1$ and we recover with satisfaction the same behaviour as featured by the simplified expression (2.11). This property provides the reason why the antiproton flux of figure 18 decreases like a power law above an energy of a few tens of GeV. If the scaling violations of the differential production cross section $d \sigma / d E_{\mathrm{p}}(\mathrm{pH} \rightarrow \overline{\mathrm{p}})$ of relation $(2.15)$ were negligible, the secondary source term $q_{\overline{\mathrm{p}}}^{\mathrm{sec}}(r, E)$ would have the same energy dependence as the impinging cosmic ray flux $\Phi_{\mathrm{p}}=\beta_{\mathrm{p}} \psi_{\mathrm{p}} / 4 \pi \propto E^{-\gamma}$. Because $G_{\overline{\mathrm{p}}}$ is proportional to $1 / K(E) \propto E^{-\delta}$ at high energies, we expect the secondary antiproton flux to have a typical spectral behaviour like

$$
G_{\overline{\mathrm{p}}} \times \Phi_{\mathrm{p}} \propto E^{-\gamma-\delta} .
$$

The cosmic ray proton and helium fluxes can be borrowed from [51] where a fit of the BESS [63] and AMS [64] data is proposed. The spectral index $\gamma$ is found to be equal to 2.72 for protons and to 2.74 for alpha particles. Would hadronic interactions be scale invariant, the antiproton flux of figure 18 , for which $\delta=0.7$, would drop like $\sim E^{-3.4}$. The actual spectrum is slightly harder with an $E^{-3.3}$ energy dependence.

The spallation of interstellar $\mathrm{H}$ and $\mathrm{He}$ by cosmic ray primaries - essentially protons and alpha particles - produce an irreducible background of secondary antiprotons inside which the signature from putative DM species is swamped. The precise determination of this background is crucial in order to disentangle a possible WIMP signal. The semi-analytic treatment of cosmic ray propagation which has been discussed above and which is based on the Bessel expansion (2.12) is a convenient framework to derive the theoretical uncertainties associated to the various parameters at stake, namely $K_{0}, \delta, V_{a}, V_{C}$ and the $\mathrm{DH}$ half-thickness $L$. The space of these propagation parameters has been extensively scanned [50] in order to select the allowed regions where the predictions on $\mathrm{B} / \mathrm{C}$ - a typical cosmic ray secondary to primary ratio - match the observations. Several hundreds of different propagation models have survived that crucial test. The propagation parameters are thus only loosely constrained by the cosmic ray nuclei abundances so far observed. The same conclusion has been reached independently by [65] with the help of a fully numerical code [66] in which the convective wind $V_{C}$ increases linearly with vertical height $z$. However, the $\mathrm{B} / \mathrm{C}$ ratio could not be accounted for when both galactic convection and diffusive reacceleration were implemented at the same time, a problem which our Bessel treatment does not encounter. The yellow band presented in figure 19 is the envelope of the secondary antiproton spectra computed with the set of $\sim 1,600$ different propagation models found in [50] to pass the $\mathrm{B} / \mathrm{C}$ test. This band comprises the theoretical uncertainty in the determination of the secondary antiproton flux. It is confined by the MIN and MAX configurations of table 4. As a first observation, notice how narrow the uncertainty strip is between $\sim 10$ and $100 \mathrm{GeV}$. The PAMELA and AMS-02 collaborations 

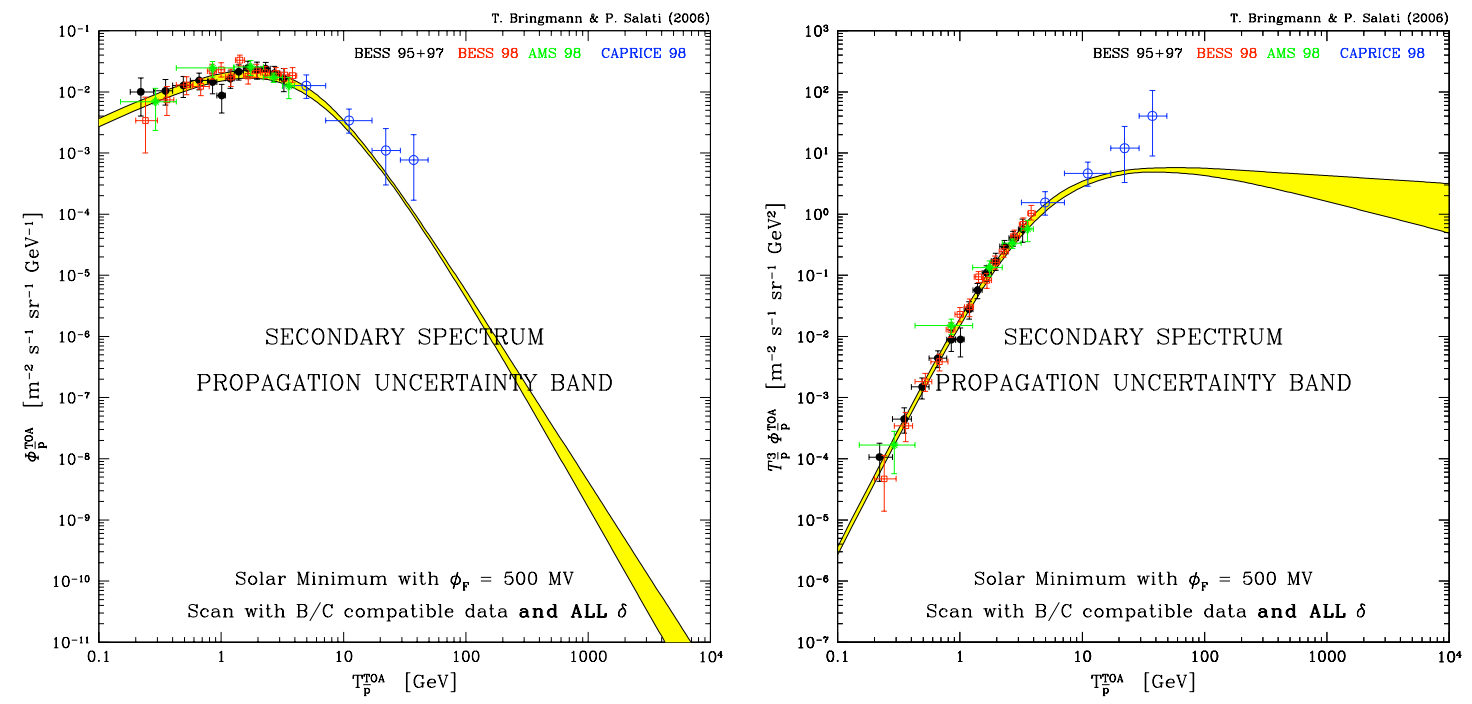

Figure 19: Theoretical uncertainties in the secondary flux of antiprotons, taking into account the whole range of propagation parameters that is allowed by the existing $\mathrm{B} / \mathrm{C}$ data, again featured together with the existing low-energy data. In the right panel, the product $T_{\overline{\mathrm{p}}}^{3} \Phi_{\overline{\mathrm{p}}}$ has been plotted in order to better illustrate the expected near $T_{\overline{\mathrm{p}}}^{-3}$ scaling of the flux at high energies. Figures from [53].

will thus be able to highlight even small spectral deviations in that energy range. Above $\sim 100$ $\mathrm{GeV}$, the yellow band widens as a result of the energy dependence of the diffusion coefficient $K$. From the $\mathrm{B} / \mathrm{C}$ analysis, the spectral index $\delta$ may take any value between 0.46 and 0.85 . Its spread $\Delta \delta=0.4$ thus translates into a factor of $10^{0.8} \sim 6$ of uncertainty on the secondary antiproton flux at $10 \mathrm{TeV}$, two decades above the energy where the yellow strip is still the thinnest. This expectation is actually confirmed in the right panel of figure 19, where we can read off a ratio of 1 to 6 at $10 \mathrm{TeV}$ between the minimal and maximal antiproton flux expectations. This large uncertainty in the secondary antiproton background at $\mathrm{TeV}$ energies may look depressing. One should keep in mind, however, that PAMELA and AMS-02 will considerably improve the measurements of the cosmic ray nuclei abundances with a determination of the $\mathrm{B} / \mathrm{C}$ ratio to a better accuracy and over a wider energy range than available so far. This will translate into improved constraints on the propagation parameters and eventually into a thinner uncertainty strip in the panels of figure 19 . The antiproton spectrum itself will also be measured up to a few $\mathrm{TeV}$ in the case of AMS-02. Once it is compared to the cosmic ray proton and helium fluxes, the spectral index $\delta$ should be better determined. Finally, we expect the LHC to improve the accuracy of the antiproton production cross sections of the various nucleus-nucleus interactions at stake.

The antiproton signal from annihilating DM particles leads to a primary component directly produced throughout the DH. It depends on many unknown ingredients. To commence, the WIMP annihilation cross section $\left\langle\sigma_{\mathrm{ann}} v\right\rangle$ at freeze-out is related to the WIMP relic abundance by

$$
\Omega_{\chi} h^{2} \simeq \frac{3 \times 10^{-27} \mathrm{~cm}^{3} \mathrm{~s}^{-1}}{\left\langle\sigma_{\mathrm{ann}} v\right\rangle} .
$$

Since today the cosmological abundance of dark matter is $\Omega_{\mathrm{DM}} \sim 0.21$ while the Hubble constant has converged toward $h \sim 0.7$, we infer a typical WIMP annihilation cross section at decoupling 
of order $3 \times 10^{-26} \mathrm{~cm}^{3} \mathrm{~s}^{-1}$. In supersymmetry, the DM candidate is a neutral Majorana fermion - generically called the neutralino - whose annihilation cross section is s-wave suppressed, hence a smaller value today than in the early universe when the temperature of the primordial plasma represented a sizable fraction of the WIMP mass. This s-wave suppression depends on the supersymmetric model under consideration and is in general large enough to significantly inhibit the antiproton DM signal. Notice that some supersymmetric configurations are nevertheless associated to large cross sections today. The WIMP annihilation at decoupling is in general so enhanced that the WIMP relic abundance is too much depleted to yield detectable annihilation signatures. As discussed below, the antiproton signal depends also sensitively on the galactic DM profile and the flux at the Earth is influenced by cosmic ray propagation throughout the DH. As is clear in figure 19, the antiproton observations are already well explained by a pure secondary component arising from the spallation of cosmic ray nuclei on the interstellar gas of the galactic disc. The CAPRICE data vaguely point toward a possible antiproton excess above $10 \mathrm{GeV}$, a region which will be soon investigated by PAMELA and AMS-02. The window extending above $10 \mathrm{GeV}$ up to a few $\mathrm{TeV}$ could be actually surprising. We will thus focus hereafter on heavy WIMP candidates whose annihilations can generate high-energy antiprotons. The models which we have selected for this analysis are summarized in table 5 where both supersymmetric and extra dimension configurations are featured. They fall into three main categories.

\begin{tabular}{|l||c|c|c|c|c|c|c|c|c|c|c|c|}
\hline DM model & $m_{\chi}$ & $\left\langle\sigma_{\mathrm{ann}} v\right\rangle$ & $t \bar{t}$ & $b \bar{b}$ & $c \bar{c}$ & $s \bar{s}$ & $u \bar{u}$ & $d \bar{d}$ & $Z Z$ & $W^{+} W^{-}$ & $H H$ & $g g$ \\
\hline \hline LSP 1.0 & 1.0 & 0.46 & - & - & - & - & - & - & - & 100 & - & - \\
LKP 1.0 & 1.0 & 1.60 & 10.9 & 0.7 & 11.1 & 0.7 & 11.1 & 0.7 & 0.5 & 1.0 & 0.5 & 0.5 \\
LSP 1.7 & 1.7 & 102 & - & - & - & - & - & - & 20.1 & 79.9 & - & - \\
LKP 1.7 & 1.7 & 0.55 & 11.0 & 0.7 & 11.1 & 0.7 & 11.1 & 0.7 & 0.5 & 0.9 & 0.5 & 0.5 \\
\hline
\end{tabular}

Table 5: Benchmark models for studying possible primary contributions to the antiproton spectrum at high energies. The DM particle mass $m_{\chi}$ is expressed in $\mathrm{TeV}$ whereas $\left\langle\sigma_{\mathrm{ann}} v\right\rangle$ denotes the annihilation rate in units of $10^{-26} \mathrm{~cm}^{3} \mathrm{~s}^{-1}$. The remaining columns give the branching ratios into the annihilation channels relevant for $\bar{p}$ production (in percent). The corresponding values are typical for high Higgsino (LSP 1.0) and Wino (LSP 1.7) fractions of the neutralino. For the latter, the non-perturbative expressions are taken from [67] while for the former the annihilation cross section of a pure anti-symmetric Higgsino into $W$ bosons has been calculated neglecting other annihilation channels. In the case of the LKP - the lightest Kaluza-Klein particle of models with universal extra dimensions (UED) [68] - the quoted values for the branching ratios as well as for $\left\langle\sigma_{\mathrm{ann}} v\right\rangle m_{\chi}^{2}$ are actually very insensitive to the parameters of the model $[69,70]$.

(i) LSP 1.0 - In the framework of supersymmetry, the lightest stable supersymmetric particle (LSP) provides an excellent dark matter candidate [16]. In most models, it is given by the lightest neutralino, which is a linear combination of the superpartners of the gauge and Higgs fields

$$
\chi \equiv \tilde{\chi}_{1}^{0}=N_{11} \tilde{B}+N_{12} \tilde{W}^{3}+N_{13} \tilde{H}_{1}^{0}+N_{14} \tilde{H}_{2}^{0}
$$

While the neutralino is often a gaugino, with a large Bino fraction and a mass of a couple of hundred $\mathrm{GeV}$ or less, the hyperbolic branch/focus point region of minimal supergravity (mSUGRA) typically exhibits very heavy neutralinos with a large Higgsino fraction [71]. From the requirement 
that Higgsinos should give the right relic density, their mass has to be around $1 \mathrm{TeV}$ [72]. For these high masses, the neutralino is an almost pure anti-symmetric combination of the two neutral Higgsino states - in which case the annihilation cross section into $Z$ as well as into Higgs boson pairs vanishes exactly and that into quarks is usually heavily suppressed by multi-TeV squark masses in the propagator. For these reasons the annihilation into $W$ bosons typically dominates.

(ii) LSP 1.7 - Another interesting situation arises in the case of a neutralino that is almost a pure Wino, as expected for example in anomaly mediated supersymmetry breaking (AMSB) scenarios [73]. For Winos, the preferred mass from relic density requirements is peaked at about $1.7 \mathrm{TeV}$ [72]. Non-perturbative binding energy effects then result in greatly enhanced annihilation cross sections today when the neutralinos have very small galactic velocities [67]. In this limit, heavy Winos annihilate almost exclusively into gauge bosons. Notice that the annihilation into photons is also significantly enhanced with respect to the perturbative result, leading to promising prospects for an indirect detection in terms of gamma rays $[67,74,75]$.

(iii) LKP 1.0 \& 1.7 - The last example of a TeV scale dark matter candidate we want to consider here is that of the lightest Kaluza-Klein particle (LKP) in models with universal extra dimensions (UED) [68], where all standard model fields are allowed to propagate in a higher-dimensional bulk. After compactification of the internal space, these additional degrees of freedom appear as towers of new, heavy states in the effective four-dimensional theory. The stability of the lightest of these states - the LKP - is guaranteed by the existence of an internal $Z_{2}$ symmetry called KK parity that derives from higher-dimensional translational invariance. Taking into account radiative corrections to the KK masses, the LKP is expected to be well approximated by the $B^{(1)}$, the first KK excitation of the weak hypercharge boson [76]. Detailed relic density calculations show that it can account for the required dark matter density if the compactification scale - and thus the $B^{(1)}$ mass - lies in the range $0.6 \lesssim m_{B^{(1)}} \lesssim 1.4 \mathrm{TeV}$ [77], mainly depending on the standard model Higgs mass. Deviations from the minimal scheme for calculating the radiative mass spectrum weaken the upper bound on the compactification scale to about 2 or $3 \mathrm{TeV}$ [78]. The main annihilation channels of the $B^{(1)}$ that are relevant for antiproton production are those into quark pairs. They amount to about $35 \%$ in total whereas the annihilation into gauge and Higgs bosons is of the order of $1 \%$ each and thus subdominant.

\begin{tabular}{|l||c|c|c||c|c|}
\hline Halo model & $\alpha$ & $\beta$ & $\gamma$ & $\rho_{\mathrm{s}}\left[10^{6} M_{\odot} \mathrm{kpc}^{-3}\right]$ & $r_{\mathrm{s}}[\mathrm{kpc}]$ \\
\hline \hline Cored isothermal [2] & 2 & 2 & 0 & 7.90 & 4 \\
NFW 97 [79] & 1 & 3 & 1 & 5.38 & 21.75 \\
Moore 04 [80] & 1 & 3 & 1.16 & 2.54 & 32.62 \\
Moore 99 [81] & 1.5 & 3 & 1.5 & 1.06 & 34.52 \\
\hline
\end{tabular}

Table 6: Parameters in equation (2.34) for the halo models considered in figure 21. The scale radius $r_{\mathrm{s}}$ and density $\rho_{\mathrm{s}}$ are strongly correlated with the virial mass of the Galaxy [82] and the values are borrowed from [83] for the Milky Way. In the case of the Moore 99 profile, DM self-annihilations set an upper bound to the maximal possible density, hence the existence of a cutoff radius inside which the DM density $\rho\left(\mathbf{x}_{S}\right)$ is assumed to be constant [84]. When the DM distribution is cuspy - for $\gamma \geq 1-$ the divergence at the galactic center is smoothed according to the prescription of [53]. 

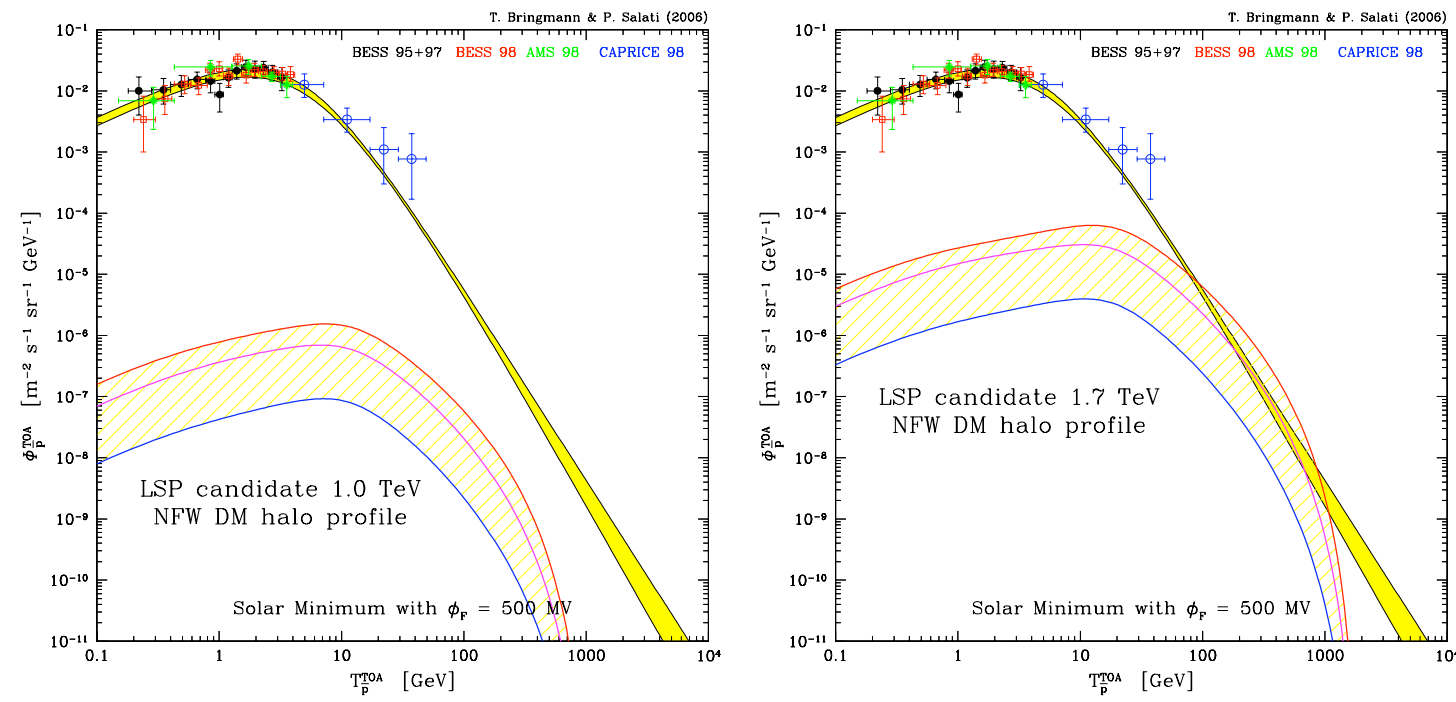

Figure 20: The primary flux in antiprotons is compared to the background in secondaries for the supersymmetric models LSP 1.0 and 1.7 of table 5. An NFW dark matter halo is assumed. The cosmic ray propagation parameters are varied from the MIN (blue) to the MAX (red) configurations of table 4. The magenta solid line corresponds to the model (MED) which provides the best fit to the $\mathrm{B} / \mathrm{C}$ measurements $[50,52]$. Figures from [53].
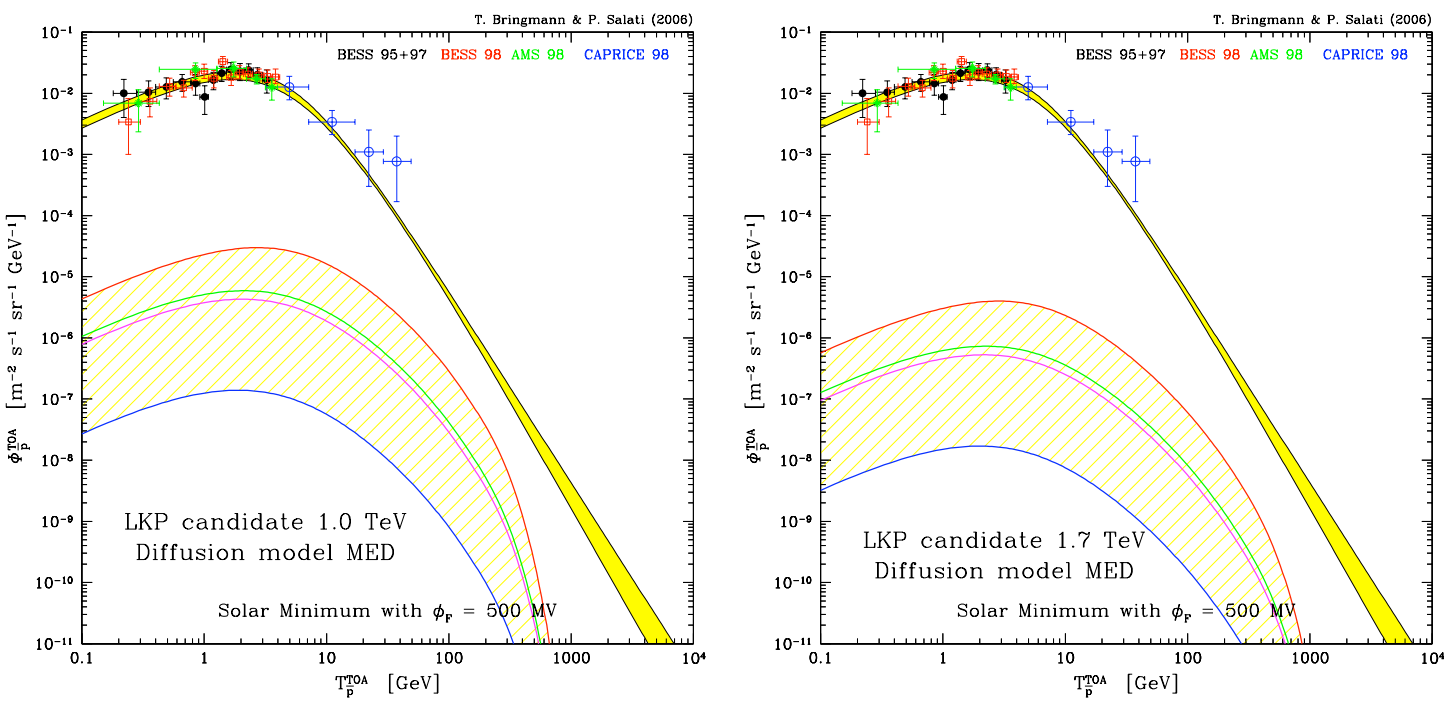

Figure 21: Same as figure 20 for the extra dimension benchmark models specified in table 5. From bottom to top, the different curves correspond respectively to the isothermal sphere (blue), NFW (magenta), Moore 04 (green) and Moore 99 (red) DM halo profiles. For the diffusion parameters, we adopt the MED configuration of table 4. Notice the important spread in the magnitude of the antiproton DM signal. The isothermal sphere model has been borrowed from [83] and features an extreme situation where the DM solar neighborhood density is unusually small. Figures from [53].

The antiproton DM signals of the WIMP benchmark models of table 5 are featured by the magenta solid curves of figures 20 and 21 . These primary fluxes have been derived with the MED set of ta- 
ble 4 and assuming an NFW DM halo profile. Unfortunately, the expected primary components are usually smaller than the background fluxes in secondary antiprotons, with the striking exception of the $1.7 \mathrm{TeV}$ Wino where the resonantly enhanced annihilation cross section may allow for a spectacular signal in the range of a few $100 \mathrm{GeV}$ already for very conservative assumptions about the DM distribution. As claimed by [85], Wino DM exhibits very promising observational prospects in terms of primary contributions to the antiproton flux. The dependence of the primary antiproton DM flux on the cosmic ray diffusion parameters is presented in figure 20. The yellow hatched band is delineated by the MIN (blue) and MAX (red) configurations of table 4. The dependence on the DH model is rather strong - although the $\mathrm{B} / \mathrm{C}$ constraints basically fix the secondary antiproton flux. This effect can be attributed $[51,52]$ to the fact that primary and secondary antiprotons mainly probe different regions of the halo. It clearly illustrates the need for cosmic ray data that are both more accurate and span a larger energy range, each of which would greatly increase the predictability for primary contributions to the antiproton flux. The influence of the DM halo profile is analyzed in figure 21 where the various possibilities of table 6 are featured. The DM galactic distribution is assumed to be spherical with a dependence on galactocentric distance $r$ parameterized by

$$
\rho(r)=\rho_{\mathrm{s}}\left(\frac{r_{\mathrm{s}}}{r}\right)^{\gamma}\left\{1+\left(\frac{r}{r_{\mathrm{s}}}\right)^{\alpha}\right\}^{(\gamma-\beta) / \alpha} .
$$

The four different profiles of table 6 basically span the whole range of reasonable halo models with respect to indirect dark matter detection prospects. Here again, the uncertainty is quite significant although the isothermal sphere model of [83] - which provides the lowest (blue) primary fluxes is somewhat extreme as it yields an unusually small DM solar neighborhood density.

Finally, let us address the question whether the next generation of experiments will be able to distinguish between the WIMP models presented here. To this end, we consider in figure 22 for each model a boost factor that normalizes the maximal deviation from the secondary flux to that of the $1.7 \mathrm{TeV}$ Wino case. At high energy, the precision of cosmic ray flux measurements is essentially limited by statistics. For comparison, the statistical error after 3 years of data sampling by PAMELA and AMS-02 have also been included - provided that these experiments would measure an antiproton spectrum as induced by LSP annihilations. A heavy Wino species can easily be distinguished from the background - even when taking into account the full uncertainty in the spectrum of secondary antiprotons - already by PAMELA. No boost factor is necessary in that case. However, in order to discriminate the spectra of the other benchmark DM candidates at a similar confidence level, artificial enhancement factors must be invoked with values from about 150 up to 500. When it comes to the actual discrimination between different DM candidates, the prospects are less promising. Given the current uncertainty in the secondary flux, as well as the expected statistical errors in the data, neither PAMELA nor AMS-02 will be able to distinguish between different types of annihilating DM species - i.e. LSP versus LKP. A determination of the WIMP mass, on the other hand, will be possible to a certain extent - at least when a clear drop in the spectrum becomes visible. A certain degeneracy between the WIMP type and its mass appears in the spectrum, putting a principle limit on the accuracy of any possible mass determination. For a given mass, the LSP produces an annihilation spectrum that is very similar to that of an LKP, apart from being slightly shifted to higher energies. An LKP with a somewhat enhanced mass - say by 

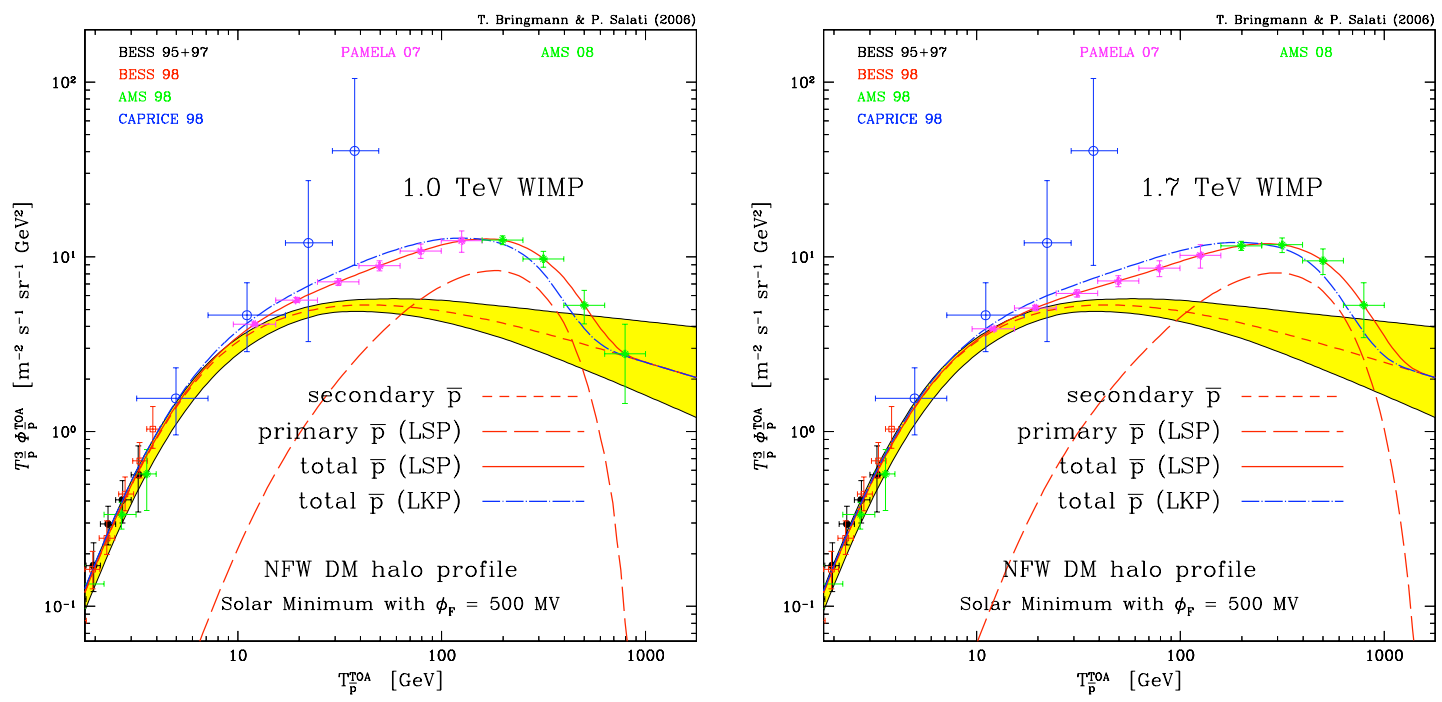

Figure 22: The yellow band features the expected antiproton background for the full range of allowed diffusion parameters. In the left (right) panel, the primary and total fluxes for the $1 \mathrm{TeV}(1.7 \mathrm{TeV})$ benchmark WIMP models of table 5 have been added. An NFW halo model and the MED set of cosmic ray diffusion parameters have been selected here. For illustration - and to better compare these models - we have adopted a boost factor of $2(1050,330,270)$ for the case of a LSP 1.7 (LKP 1.7, LSP 1.0, LKP 1.0) DM candidate. For the LSP, we also include the expected statistical error after 3 years of data sampling by PAMELA and AMS-02, respectively. Figures from [53].

about $10 \%$ - would therefore feature a spectrum that is almost indistinguishable from that of the LSP.

To conclude this section, let us point out that apart from the case of the heavy Wino for which the annihilation cross section is resonantly enhanced, prospects for observing the antiproton DM signal are poor. That is why a boost factor is generally introduced in the litterature in order to enhance the primary fluxes and make them observable. This procedure is justified by the existence of substructures in the DM galactic distribution inside which the WIMPs are clumped. That hand waving argument is scrutinized in section 3 where boost factors are shown to be energy dependent and subject to strong statistical uncertainties.

\subsection{The positron signal}

In the case of positrons and electrons, the master equation (2.5) describing the propagation of cosmic rays throughout the DH is dominated by space diffusion and energy losses. Above a few $\mathrm{GeV}$, synchrotron radiation in the galactic magnetic fields as well as inverse Compton scattering on stellar light and on $\mathrm{CMB}$ photons dominate, hence the positron loss rate

$$
b^{\text {loss }}(E)=\langle\dot{E}\rangle=-\frac{E^{2}}{E_{0} \tau_{E}} .
$$

The energy of reference $E_{0}$ is set equal to $1 \mathrm{GeV}$ while the typical energy loss time is $\tau_{E}=10^{16} \mathrm{~s}$. The master equation for positron propagation simplifies into

$$
-K \Delta \psi+\partial_{E}\left\{b^{\text {loss }}(E) \psi\right\}=q(\mathbf{x}, E) .
$$


For the energies under consideration - above $\sim 0.1 \mathrm{GeV}-$ positrons are ultra-relativistic and the rigidity $\mathscr{R}$ is proportional to the energy $E$. The space diffusion coefficient can be expressed as

$$
K(\varepsilon)=K_{0} \varepsilon^{\delta},
$$

where $\varepsilon$ denotes the ratio $E / E_{0}$.

Exercise $\mathbf{n}^{0}$ 2-d - Level [1] : Taking into account relations (2.35) and (2.37), derive the positron propagation equation

$$
K_{0} \varepsilon^{\delta} \Delta \psi+\frac{\partial}{\partial \varepsilon}\left\{\frac{\varepsilon^{2}}{\tau_{E}} \psi\right\}+q=0 .
$$

Equation (2.38) may be solved with Baltz \& Edsjö [86] clever trick which consists in translating the energy $E$ into the pseudo-time

$$
\tilde{t}(E)=\tau_{E}\left\{v(E)=\frac{\varepsilon^{\delta-1}}{1-\delta}\right\} .
$$

In this formalism, the energy losses which positrons experience boil down to a mere evolution in the pseudo-time $\tilde{t}$.

Exercise $\mathbf{n}^{0}$ 2-e - Level [1] : Show that the propagation equation (2.38) simplifies into the wellknown heat equation

$$
\frac{\partial \tilde{\psi}}{\partial \tilde{t}}-K_{0} \Delta \tilde{\psi}=\tilde{q}(\mathbf{x}, \tilde{t})
$$

The space and energy positron density is now $\tilde{\psi}=\varepsilon^{2} \psi$ whereas the positron production rate has become $\tilde{q}=\varepsilon^{2-\delta} q$. Notice that both $\tilde{\psi}$ and $\tilde{q}$ have the same dimensions as before because $\varepsilon$ is dimensionless.

Without any space boundary condition, the solution of equation (2.40) is straightforward. A droplet of heat deposited at the origin of the coordinates at pseudo-time $\tilde{t}_{S}=0$ is described by the pointlike source term

$$
\tilde{q}\left(\mathbf{x}_{S}, \tilde{t}_{S}\right)=\delta^{3}\left(\mathbf{x}_{S}\right) \delta\left(\tilde{t}_{S}\right)
$$

The subsequent diffusion in infinite $3 \mathrm{D}$ space results into the density $\tilde{\psi}$ at position $\mathbf{x}$ and pseudotime $\tilde{t}$ given by the well-known Green function

$$
\tilde{\psi}(\mathbf{x}, \tilde{t}) \equiv \tilde{G}(\mathbf{x}, \tilde{t} \leftarrow \mathbf{0}, 0)=\theta(\tilde{t})\left\{4 \pi K_{0} \tilde{t}\right\}^{-3 / 2} \exp \left\{-\frac{r^{2}}{4 K_{0} \tilde{t}}\right\}
$$

where $r \equiv|\mathbf{x}|$ and $\theta$ is Heaviside's step function. The general solution of equation (2.40) can be written in terms of the Green function $\tilde{G}$ as an integral over pseudo-time and space

$$
\tilde{\psi}(\mathbf{x}, \tilde{t})=\int_{\tilde{t}_{S}=0}^{\tilde{t}_{S}=\tilde{t}} d \tilde{t}_{S} \int_{\mathrm{DH}} d^{3} \mathbf{x}_{S} \tilde{G}\left(\mathbf{x}, \tilde{t} \leftarrow \mathbf{x}_{S}, \tilde{t}_{S}\right) \tilde{q}\left(\mathbf{x}_{S}, \tilde{t}_{S}\right)
$$


We are lead to express the density of positrons resulting from their transport within the Milky Way down to $\mathbf{x}$ as the convolution

$$
\psi_{e^{+}}(\mathbf{x}, E)=\int_{E_{S}=E}^{E_{S}=+\infty} d E_{S} \int_{\mathrm{DH}} d^{3} \mathbf{x}_{S} G_{e^{+}}\left(\mathbf{x}, E \leftarrow \mathbf{x}_{S}, E_{S}\right) q_{e^{+}}\left(\mathbf{x}_{S}, E_{S}\right)
$$

recovering thus the generic expression (2.6). The positron propagator $G_{e^{+}}\left(\mathbf{x}, E \leftarrow \mathbf{x}_{S}, E_{S}\right)$ stands for the probability for a particle injected at $\mathbf{x}_{S}$ with the energy $E_{S}$ to reach the location $\mathbf{x}$ with the degraded energy $E \leq E_{S}$.

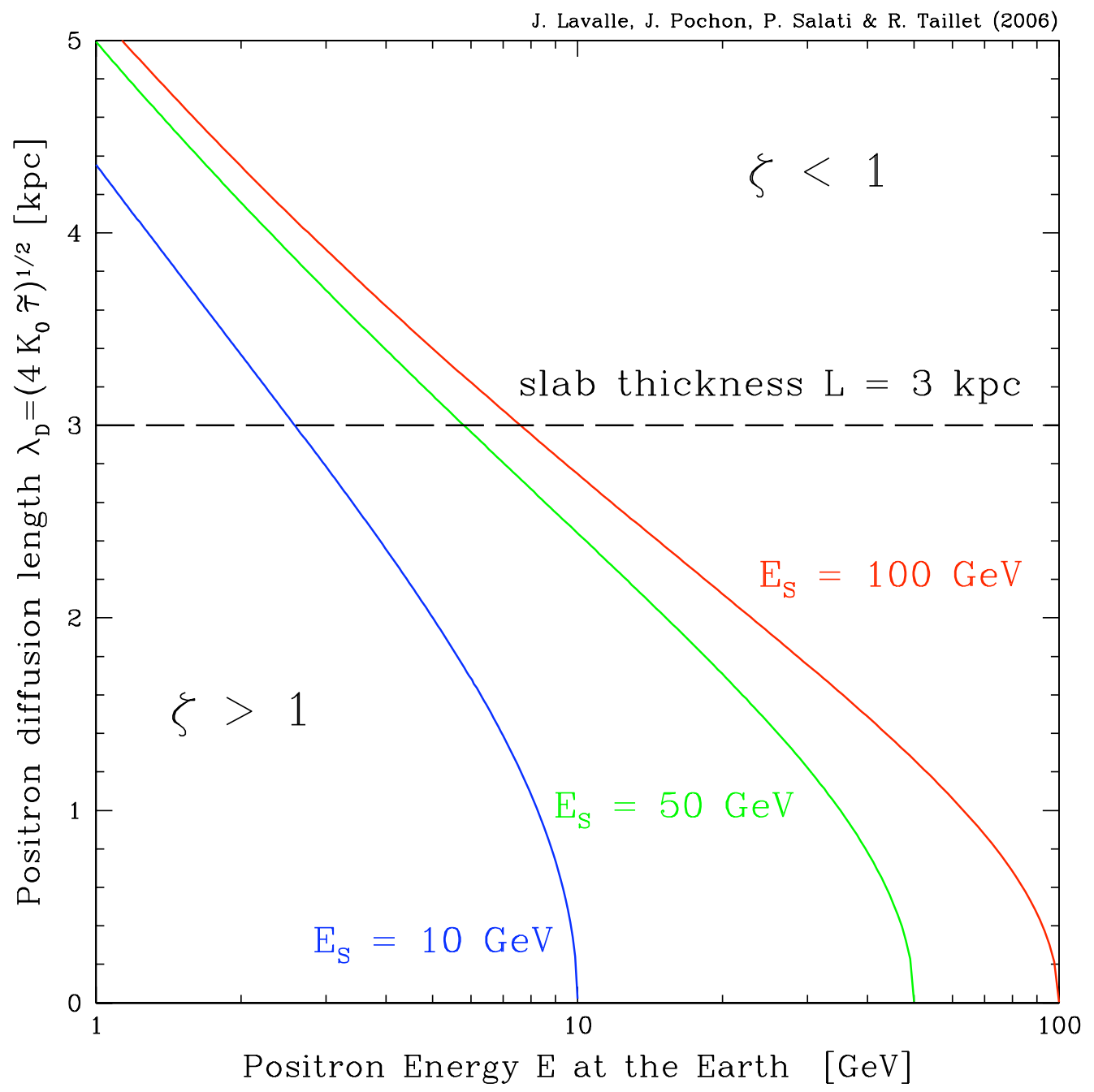

Figure 23: The positron diffusion length $\lambda_{\mathrm{D}}$ is featured as a function of the positron energy $E$ at the Earth for three different values of the energy $E_{S}$ at the source. In this illustration, the space diffusion coefficient at $1 \mathrm{GV}$ has been set equal to $K_{0}=3 \times 10^{27} \mathrm{~cm}^{2} \mathrm{~s}^{-1}$ with a spectral index $\delta=0.6$. The long-dashed horizontal line corresponds to a diffusion length $\lambda_{\mathrm{D}}$ equal to the half-thickness $L=3 \mathrm{kpc}$ of the DH. Below that limit, positron propagation is not sensitive to the vertical boundaries and the $3 \mathrm{D}$ approximation (2.46) is valid. This regime corresponds to large values of the parameter $\zeta \equiv L^{2} / \lambda_{\mathrm{D}}{ }^{2}$. Figure from [87]. 
It is proportional to the heat Green function $\tilde{G}$ through

$$
G_{e^{+}}\left(\mathbf{x}, E \leftarrow \mathbf{x}_{S}, E_{S}\right)=\frac{\tau_{E}}{E_{0} \varepsilon^{2}} \tilde{G}\left(\mathbf{x}, \tilde{t} \leftarrow \mathbf{x}_{S}, \tilde{t}_{S}\right)
$$

where the connection between the energy $E$ and pseudo-time $\tilde{t}$ is given by relation (2.39).

Exercise $\mathbf{n}^{0}$ 2-f - Level [1] : Show that in the 3D limit of an infinite DH, the heat Green function connecting the source $\mathbf{x}_{S}$ to the Earth is the Gaussian distribution

$$
\tilde{G}\left(\mathbf{x}_{\odot}, \tilde{t} \leftarrow \mathbf{x}_{S}, \tilde{t}_{S}\right)=\left\{\frac{1}{4 \pi K_{0} \tilde{\tau}}\right\}^{3 / 2} \exp \left\{-\frac{r_{\oplus}^{2}}{4 K_{0} \tilde{\tau}}\right\}
$$

where $\tilde{\tau}=\tilde{t}-\tilde{t}_{S}$ is the typical duration - including the diffusion process - over which the positron energy decreases from $E_{S}$ to $E$. The distance between the Earth and the source is denoted by

$$
r_{\oplus}=\left\{\left(x_{\odot}-x_{S}\right)^{2}+\left(y_{\odot}-y_{S}\right)^{2}+\left(z_{\odot}-z_{S}\right)^{2}\right\}^{1 / 2} .
$$

The concept of positron horizon is based on the Gaussian distribution (2.46) which is roughly constant within a sphere of radius

$$
\lambda_{\mathrm{D}}=\sqrt{4 K_{0} \tilde{\tau}},
$$

and decreases sharply outside. So does the positron Green function $G_{e^{+}}$. The so-called positron sphere - whose center is at the Earth where the observer stands - delineates thus the region of the diffusive halo from which positrons predominantly originate. The typical diffusion length $\lambda_{D}$ gauges how far particles produced with the energy $E_{S}$ travel before being detected at the energy $E$. It encodes at the same time the energy loss processes and the diffusion throughout the magnetic fields of the Galaxy. A rapid inspection of equation (2.39) shows that $\lambda_{D}$ increases as the detected energy $E$ decreases, except for energies $E_{S}$ at the source very close to $E$. The positron sphere is therefore fairly small at high energies - say above $\sim 100 \mathrm{GeV}$ - whereas it spreads over several kiloparsecs below $10 \mathrm{GeV}$. The diffusion length $\lambda_{\mathrm{D}}$ is featured in figure 23 as a function of $E$ for three different values of the energy at the source. In the case where $E_{S}=100 \mathrm{GeV}$, it exceeds the half-thickness $L$ below an energy of $\sim 8 \mathrm{GeV}$.

The diffusive halo inside which cosmic rays propagate before escaping into the intergalactic medium is actually finite. Without any boundary condition, the propagator $\tilde{G}$ would be given by the $3 \mathrm{D}$ relation (2.46). However, cosmic rays may escape outside what has been modeled initially as a leaky box and $\tilde{G}$ should account for that effect. In spite of the boundaries at $r=R \equiv 20 \mathrm{kpc}$, we can still decide that cosmic ray diffusion is not limited along the radial direction and that it operates as if it took place inside an infinite horizontal slab with half-thickness $L$. Sources located beyond the radius $R$ should be obviously disregarded since the convolution (2.44) is only performed over the $\mathrm{DH}$. Because their energy is rapidly degraded as they propagate, positrons are produced close to where they are observed. Neglecting the effect of radial boundaries on the propagator $G_{e^{+}}$turns out to be a fair approximation [88] because positrons do not originate from far away on average. Even in the case of antiprotons for which the galactic propagation range is significantly larger than 
for positrons, the effects of radial boundaries down at the Earth are not significant insofar as cosmic ray species tend to leak above and beneath the diffusive halo at $z= \pm L$ instead of traveling a long distance along the galactic disc. The infinite slab hypothesis allows the radial and vertical directions to be disentangled and the reduced propagator $\tilde{G}$ may be expressed as

$$
\tilde{G}\left(\mathbf{x}, \tilde{t} \leftarrow \mathbf{x}_{S}, \tilde{t}_{S}\right)=\frac{\theta(\tilde{\tau})}{4 \pi K_{0} \tilde{\tau}} \exp \left\{-\frac{r^{2}}{4 K_{0} \tilde{\tau}}\right\} \tilde{V}\left(z, \tilde{t} \leftarrow z_{S}, \tilde{t}_{S}\right)
$$

where $\tilde{\tau}=\tilde{t}-\tilde{t}_{S}$ as before. The radial distance between the source $\mathbf{x}_{S}$ and the point $\mathbf{x}$ of observation is defined as

$$
r=\left\{\left(x-x_{S}\right)^{2}+\left(y-y_{S}\right)^{2}\right\}^{1 / 2}
$$

Exercise $\mathbf{n}^{0}$ 2-g - Level [1] : Should cosmic ray transport be free along the vertical direction, show that the propagator $\tilde{V}$ would be given by the $1 D$ solution $\mathscr{V}_{1 D}$ to the diffusion equation (2.40)

$$
\tilde{V}\left(z, \tilde{t} \leftarrow z_{S}, \tilde{t}_{S}\right) \equiv \mathscr{V}_{1 D}\left(z, \tilde{t} \leftarrow z_{S}, \tilde{t}_{S}\right)=\frac{\theta(\tilde{\tau})}{\sqrt{4 \pi K_{0} \tilde{\tau}}} \exp \left\{-\frac{\left(z-z_{S}\right)^{2}}{4 K_{0} \tilde{\tau}}\right\} .
$$

The vertical boundary conditions definitely need to be implemented. Wherever the source inside the slab, the positron density vanishes at $z= \pm L$.

(i) A first approach relies on the method of the so-called electrical images and has been discussed in [86]. Any point-like source inside the slab is associated to the infinite series of its multiple images through the boundaries at $z= \pm L$ which act as mirrors. The $\mathrm{n}$-th image is located at

$$
z_{n}=2 L n+(-1)^{n} z_{S},
$$

and has a positive or negative contribution depending on whether $n$ is an even or odd number. When the diffusion time $\tilde{\tau}$ is small, the 1D solution (2.51) is a quite good approximation. The relevant parameter is actually

$$
\zeta=\frac{L^{2}}{4 K_{0} \tilde{\tau}} \equiv \frac{L^{2}}{\lambda_{\mathrm{D}}^{2}}
$$

and in the regime where it is much larger than 1, the propagation is insensitive to the vertical boundaries. On the contrary, when $\zeta$ is much smaller than 1, a large number of images need to be taken into account in the sum

$$
\tilde{V}\left(z, \tilde{t} \leftarrow z_{S}, \tilde{t}_{S}\right)=\sum_{n=-\infty}^{+\infty}(-1)^{n} \mathscr{V}_{1 D}\left(z, \tilde{t} \leftarrow z_{n}, \tilde{t}_{S}\right),
$$

and convergence may be a problem.

(ii) It is fortunate that a quite different approach is possible in that case. The 1D diffusion equation (2.40) actually looks like the Schrödinger equation - in imaginary time - that accounts for the behaviour of a particle inside an infinitely deep 1D potential well which extends from $z=-L$ to $z=+L$. The eigenfunctions of the associated Hamiltonian are both even

$$
\varphi_{n}(z)=\sin \left\{k_{n}(L-|z|)\right\}
$$


and odd

$$
\varphi_{n}^{\prime}(z)=\sin \left\{k_{n}^{\prime}(L-z)\right\}
$$

functions of the vertical coordinate $z$. The wave-vectors $k_{n}$ and $k_{n}^{\prime}$ are respectively defined as

$$
k_{n}=\left(n-\frac{1}{2}\right) \frac{\pi}{L} \text { (even) and } k_{n}^{\prime}=n \frac{\pi}{L} \text { (odd) . }
$$

The vertical propagator may be expanded as the series

$$
\tilde{V}\left(z, \tilde{t} \leftarrow z_{S}, \tilde{t}_{S}\right)=\sum_{n=1}^{+\infty} \frac{1}{L}\left\{e^{-\lambda_{n} \tilde{\tau}} \varphi_{n}\left(z_{S}\right) \varphi_{n}(z)+e^{-\lambda_{n}^{\prime} \tilde{\tau}} \varphi_{n}^{\prime}\left(z_{S}\right) \varphi_{n}^{\prime}(z)\right\},
$$

where the time constants $\lambda_{n}$ and $\lambda_{n}^{\prime}$ are respectively equal to $K_{0} k_{n}{ }^{2}$ and $K_{0} k_{n}^{\prime 2}$. In the regime where $\zeta$ is much smaller than 1 - for very large values of the diffusion time $\tilde{\tau}$ - just a few eigenfunctions need to be considered in order for the sum (2.58) to converge.

Exercise $\mathbf{n}^{0}$ 2-h - Level [1]: Show that the energies $E$ and $E_{S}$ always come into play in the reduced propagator $\tilde{G}$ through the diffusion length $\lambda_{\mathrm{D}}$. Justify then the expression for the positron propagator

$$
G_{e^{+}}\left(\mathbf{x}, E \leftarrow \mathbf{x}_{S}, E_{S}\right)=\frac{\tau_{E}}{E_{0} \varepsilon^{2}} \tilde{G}\left(\mathbf{x} \leftarrow \mathbf{x}_{S} ; \lambda_{\mathrm{D}}\right) .
$$

Like for antiprotons, a background of secondary positrons is produced by the spallation of the interstellar medium by impinging high-energy particles. In that respect, the Milky Way looks like a giant accelerator where cosmic rays play the role of the beam whereas the galactic disc and its gas behave as the target. The dominant mechanism is the collision of protons with hydrogen atoms at rest producing charged pions $\pi^{ \pm}$which decay into muons $\mu^{ \pm}$. The latter are also unstable and eventually lead to electrons and positrons through the chain

$$
\begin{aligned}
\mathrm{p}+\mathrm{H} \longrightarrow \mathrm{X}+\pi^{ \pm} & \\
\pi^{ \pm} \longrightarrow v_{\mu}+\mu^{ \pm} & \\
& \mu^{ \pm} \longrightarrow v_{\mu}+v_{e}+e^{ \pm} .
\end{aligned}
$$

(a) In order to obtain the differential cross section for positron production, we need first to derive the probability for a pion with energy $E_{\pi}$ to eventually decay into a positron with energy $E_{e}$. The corresponding distribution function $\mathscr{P}_{\xi}\left(E_{\pi} \rightarrow E_{e}\right)$ is comprehensively built in appendix C of [89]. We recall here the salient features of that construction. To commence, in the pion rest frame, the muon is produced isotropically with the Lorentz factor

$$
\gamma_{\mu}^{\star}=\frac{m_{\pi}^{2}+m_{\mu}^{2}}{2 m_{\pi} m_{\mu}}
$$

and the velocity $\beta_{\mu}^{\star}$. The pion moves with the Lorentz factor $\gamma_{\pi}=E_{\pi} / m_{\pi}$ in the laboratory system inside which the muon energy is distributed according to the flat probability function

$$
\mathscr{P}\left(E_{\pi} \rightarrow E_{\mu}\right)=\frac{d N_{\mu}}{d E_{\mu}}=\frac{\Theta\left(\gamma_{\mu}^{-} \leq \gamma_{\mu} \leq \gamma_{\mu}^{+}\right)}{2 m_{\mu} \beta_{\pi} \gamma_{\pi} \beta_{\mu}^{\star} \gamma_{\mu}^{\star}},
$$


where the Lorentz factors $\gamma_{\mu}^{ \pm}$are defined as

$$
\gamma_{\mu}^{ \pm}=\gamma_{\pi} \gamma_{\mu}^{\star}\left(1 \pm \beta_{\pi} \beta_{\mu}^{\star}\right)
$$

The function $\Theta$ is set to unity provided that the condition in parenthesis is fulfilled and to 0 otherwise. The isotropy of the muon production in the pion rest frame translates in the laboratory system into a flat distribution for the muon Lorentz factor $\gamma_{\mu}=E_{\mu} / m_{\mu}$ which is furthermore constrained to lie in the range extending from $\gamma_{\mu}^{-}$to $\gamma_{\mu}^{+}$. Because weak interaction are left-handed and spin is conserved, the muon is created fully polarized in the pion frame. A positively (negatively) charged pion gives rise to a left-handed muon neutrino (right-handed muon antineutrino) and accordingly to a left-handed positive (right-handed negative) muon. In the muon rest frame, the angular distribution of positrons (electrons) is asymmetric with respect to the direction along which the muon spin is aligned and toward which that particle propagates before decaying

$$
\mathscr{P}_{\xi}^{\star}\left(E_{e}^{\star}, \cos \theta^{\star}\right)=\frac{8 E_{e}^{\star 2}}{m_{\mu}^{3}}\left\{3-4 \frac{E_{e}^{\star}}{m_{\mu}}-\xi \cos \theta^{\star}\left(1-4 \frac{E_{e}^{\star}}{m_{\mu}}\right)\right\} .
$$

The parameter $\xi$ is equal to +1 for positrons and to -1 for electrons. In the derivation presented in appendix $\mathrm{C}$ of [89], the muon is also assumed to be fully polarized in the laboratory system so that the angle $\theta$ between the positron and muon momenta - as seen in that frame - is related to the above-mentioned asymmetric angle $\theta^{\star}$ by the Lorentz boost

$$
\cos \theta=\frac{\cos \theta^{\star}+\beta_{\mu}}{1+\beta_{\mu} \cos \theta^{\star}}
$$

Exercise $\mathbf{n}^{0}$ 2-i - Level [2] : Show that the positron energy $E_{e}$ as seen in the laboratory system may be derived from its muon rest frame value $E_{e}^{\star}$ by the Lorentz boost

$$
E_{e}=\gamma_{\mu} E_{e}^{\star}\left(1+\beta_{\mu} \cos \theta^{\star}\right) .
$$

Compute the Jacobian associated to the change of the variables $\left(E_{e}^{\star}, \cos \theta^{\star}\right)$ into the new set $\left(E_{e}, \cos \theta\right)$ in order to establish the identity

$$
E_{e}^{\star} d E_{e}^{\star} d \cos \theta^{\star}=E_{e} d E_{e} d \cos \theta .
$$

Deduce finally that the muon rest frame distribution function $\mathscr{P}_{\xi}^{\star}\left(E_{e}^{\star}, \cos \theta^{\star}\right)$ and its laboratory system counterpart $\mathscr{P}_{\xi}\left(E_{e}, \cos \theta\right)$ are related by

$$
\mathscr{P}_{\xi}\left(E_{e}, \cos \theta\right)=\frac{E_{e}}{E_{e}^{\star}} \mathscr{P}_{\xi}^{\star}\left(E_{e}^{\star}, \cos \theta^{\star}\right) .
$$

We readily infer that the pion to positron (or electron) transfer function $\mathscr{P}_{\xi}\left(E_{\pi} \rightarrow E_{e}\right)$ is given in the laboratory system by the convolution

$$
\mathscr{P}_{\xi}\left(E_{\pi} \rightarrow E_{e}\right)=\frac{d N_{e}}{d E_{e}}=\int_{\gamma_{\mu}^{-}}^{\gamma_{\mu}^{+}} d \gamma_{\mu} m_{\mu} \times \mathscr{P}\left(E_{\pi} \rightarrow E_{\mu}\right) \times \int_{\cos \theta_{\mathrm{L}}}^{1} d \cos \theta \mathscr{P}_{\xi}\left(E_{e}, \cos \theta\right)
$$


The angle $\theta_{\mathrm{L}}$ corresponds to a positron (or electron) energy $E_{e}^{\star}$ in the muon rest frame equal to the maximal reachable value of $m_{\mu} / 2$. If $\cos \theta_{\mathrm{L}}$ is larger than +1 , the integral over the angle $\theta$ in relation (2.69) vanishes whereas it runs from -1 to +1 whenever $\cos \theta_{\mathrm{L}}$ becomes less than -1 . At fixed muon energy $E_{\mu}$ and Lorentz factor $\gamma_{\mu}$, these situations arise when the energy $E_{e}$ in the laboratory is respectively larger than

$$
E_{e}^{+}\left(\gamma_{\mu}\right)=\frac{m_{\mu}}{2} \sqrt{\frac{1+\beta_{\mu}}{1-\beta_{\mu}}},
$$

and smaller than

$$
E_{e}^{-}\left(\gamma_{\mu}\right)=\frac{m_{\mu}}{2} \sqrt{\frac{1-\beta_{\mu}}{1+\beta_{\mu}}} .
$$

(b) In proton-proton collisions, pions can be produced in two different ways depending on the energy $E_{\mathrm{p}}$ of the incoming cosmic ray proton in the galactic frame. Below $\sim 3 \mathrm{GeV}$, one of the protons is predominantly excited to a $\Delta$ resonance which subsequently decays into a nucleon and a pion according to the reactions

$$
\begin{aligned}
\mathrm{p}+\mathrm{H} \longrightarrow \mathrm{p}+ & \Delta^{+} \\
\Delta^{+} & \longrightarrow \mathrm{p}+\pi^{0} \\
\Delta^{+} & \longrightarrow \mathrm{n}+\pi^{+} .
\end{aligned}
$$

Exercise $\mathbf{n}^{0} \mathbf{2}-\mathbf{j}$ - Level [1] : The neutral $\pi^{0}$ and charged $\pi^{ \pm}$pions may be folded into an SU(2) isospin triplet whose eigenvalues with respect to the isospin operator $T_{3}$ are given by

$$
\left(\pi^{+}, \pi^{0}, \pi^{-}\right) \equiv(1,0,-1) .
$$

The proton and the neutron are the two eigenstates of an isospin doublet and can be excited into a $\Delta$ resonance which may exist under the four different incarnations of the isospin $3 / 2$ family

$$
\left(\Delta^{++}, \Delta^{+}, \Delta^{0}, \Delta^{-}\right) \equiv\left(\frac{3}{2}, \frac{1}{2},-\frac{1}{2},-\frac{3}{2}\right)
$$

Assuming that isospin is conserved in $\Delta$ decay, show that the branching ratios for the production of neutral $\pi^{0}$ and charged $\pi^{+}$pions are respectively equal to $2 / 3$ and $1 / 3$.

In the galactic frame, the target proton is at rest and the center of mass frame (CMF) of the reaction is characterized by the Lorentz factor

$$
\gamma_{c}=\frac{\sqrt{s}}{2 m_{\mathrm{p}}}=\sqrt{\frac{E_{\mathrm{p}}+m_{\mathrm{p}}}{2 m_{\mathrm{p}}}},
$$

and the total available energy

$$
\sqrt{s}=\sqrt{2 m_{\mathrm{p}}\left(E_{\mathrm{p}}+m_{\mathrm{p}}\right)} .
$$

Reaction (2.72) leads in the CMF to an isobar energy of

$$
E_{\Delta}^{c}=\gamma_{\Delta}^{c} m_{\Delta}=\frac{s+m_{\Delta}^{2}-m_{\mathrm{p}}^{2}}{2 \sqrt{s}}
$$


To simplify the discussion, we assume that the $\Delta$ resonance is produced solely along the beam axis with an equal probability for each direction. In the galactic frame, isobars moving forward - in the same direction as the incident cosmic ray proton - and backward - in the opposite direction - are respectively associated to the Lorentz factors

$$
\gamma_{\Delta}^{ \pm}=\gamma_{c} \gamma_{\Delta}^{c}\left(1 \pm \beta_{c} \beta_{\Delta}^{c}\right)
$$

In this scheme, two values for the isobar energy are possible in the galactic system. In the $\Delta$ rest frame, reaction (2.74) generates a pion with the Lorentz factor

$$
\gamma_{\pi}^{\star}=\frac{E_{\pi}^{\star}}{m_{\pi}}=\frac{m_{\Delta}^{2}+m_{\pi}^{2}-m_{\mathrm{n}}^{2}}{2 m_{\Delta} m_{\pi}}
$$

and the velocity $\beta_{\pi}^{\star}$. In the galactic frame, pions produced by isobars moving forward have Lorentz factors in the range extending from $\gamma_{\pi, \mathrm{F}}^{-}$to $\gamma_{\pi, \mathrm{F}}^{+}$.

Exercise $\mathbf{n}^{0} \mathbf{2}-\mathbf{k}$ - Level [1] : Inspired by the derivation of relations (2.62) and (2.63), show that

$$
\gamma_{\pi, \mathrm{F}}^{ \pm}=\gamma_{\Delta}^{+} \gamma_{\pi}^{\star}\left(1 \pm \beta_{\Delta}^{+} \beta_{\pi}^{\star}\right)
$$

Repeat the same analysis for $\Delta$ particles moving backward and establish that the pion Lorentz factors in the galactic system are now limited by

$$
\gamma_{\pi, \mathrm{B}}^{ \pm}=\gamma_{\Delta}^{-} \gamma_{\pi}^{\star}\left(1 \pm \beta_{\Delta}^{-} \beta_{\pi}^{\star}\right)
$$

Derive eventually the corresponding pion energy distribution in the galactic frame

$$
\mathscr{P}_{\Delta}\left(E_{\mathrm{p}} \rightarrow E_{\pi}\right)=\frac{d N_{\pi}}{d E_{\pi}}=\frac{1}{4 m_{\pi} \beta_{\pi}^{\star} \gamma_{\pi}^{\star}}\left\{\frac{\Theta\left(\gamma_{\pi, \mathrm{B}}^{-} \leq \gamma_{\pi} \leq \gamma_{\pi, \mathrm{B}}^{+}\right)}{\beta_{\Delta}^{-} \gamma_{\Delta}^{-}}+\frac{\Theta\left(\gamma_{\pi, \mathrm{F}}^{-} \leq \gamma_{\pi} \leq \gamma_{\pi, \mathrm{F}}^{+}\right)}{\beta_{\Delta}^{+} \gamma_{\Delta}^{+}}\right\} .
$$

The convolution of the distribution functions $\mathscr{P}_{\Delta}\left(E_{\mathrm{p}} \rightarrow E_{\pi}\right)$ and $\mathscr{P}_{+}\left(E_{\pi} \rightarrow E_{e}\right)$ over the pion energy leads to the differential cross section

$$
\frac{d \sigma}{d E_{e}}\left(E_{\mathrm{p}} \rightarrow \Delta \rightarrow E_{e}\right)=\sigma\left(E_{\mathrm{p}} \rightarrow \Delta\right) \times \int \mathscr{P}_{\Delta}\left(E_{\mathrm{p}} \rightarrow E_{\pi}\right) \times d E_{\pi} \times \mathscr{P}_{+}\left(E_{\pi} \rightarrow E_{e}\right) .
$$

The total $\Delta$ production cross section $\sigma\left(E_{\mathrm{p}} \rightarrow \Delta\right)$ depends on the incoming proton energy $E_{\mathrm{p}}$ in the galactic system or alternatively on the CMF energy $\sqrt{s}$ of reaction (2.72). A parametric form can be found in [90].

(c) Above $\sim 7 \mathrm{GeV}$, the pion production (2.60) is well described in the framework of the scaling model. Various parameterizations are given in the literature [91, 92] for the Lorentz invariant (LI) cross section

$$
E_{\pi}^{\star} \frac{d^{3} \sigma}{d^{3} p_{\pi}^{\star}}=E_{\pi} \frac{d^{3} \sigma}{d^{3} p_{\pi}} \equiv \frac{1}{p_{\pi}} \frac{d^{2} \sigma}{d \Omega_{\pi} d E_{\pi}} .
$$

The latter depends on the total energy $\sqrt{s}$ available in the center of mass frame of the proton-proton collision and on the transverse $p_{\pi, \perp} \equiv p_{\pi, \perp}^{\star}$ and longitudinal $p_{\pi, \|}^{\star}$ momenta of the pion as seen, for instance, in the CMF. 
Exercise $\mathbf{n}^{0}$ 2-l - Level [1] : We would like to compute the maximal energy $E_{\pi, \max }^{\star} \equiv E_{\pi}^{0}$ which the pion may reach in the CMF for a fixed value of the total available energy $\sqrt{s}$. As shown below, this energy $E_{\pi}^{0}$ increases with $\sqrt{s}$. Thus the problem comes down to the search for the minimal value of $\sqrt{s}$ which can still accomodate a pion with the energy $E_{\pi}^{\star}=E_{\pi}^{0}$. We need therefore to identify the final state configuration whose total energy is the smallest and which contains a pion with the energy $E_{\pi}^{0}$. Reaction (2.60) gives rise to a pion and a set of particles generically denoted by $X$. In order to minimize $\sqrt{s}$, we must find the state $X$ with the fewest particles and also with the lightest species. This configuration corresponds to what the series of reactions (2.72) and (2.74) yields - i.e. a proton and a neutron. Explain then why we may write the CMF energy as

$$
\sqrt{s}=E_{\pi}^{0}+\sqrt{m_{\mathrm{p}}^{2}+k_{\mathrm{p}, \|}^{2}+k_{\mathrm{p}, \perp}^{2}}+\sqrt{m_{\mathrm{n}}^{2}+k_{\mathrm{n}, \|}^{2}+k_{\mathrm{n}, \perp}^{2}} .
$$

The components of the proton and neutron momenta that are aligned with the pion momentum are respectively denoted by $k_{\mathrm{p}, \|}$ and $k_{\mathrm{n}, \|}$. What is the value of the perpendicular components $k_{\mathrm{p}, \perp}$ and $k_{\mathrm{n}, \perp}$ that minimize the CMF energy while the longitudinal components $k_{\mathrm{p}, \|}$ and $k_{\mathrm{n}, \|}$ are kept fixed? We are now left with a configuration where the pion, proton and neuton momenta are all aligned. The parameter $\lambda$ denotes the proton to pion momenta ratio and fulfills the identities

$$
k_{\mathrm{p}, \|}=\lambda k_{\pi}^{0} \quad \text { and } \quad k_{\mathrm{n}, \|}=(1-\lambda) k_{\pi}^{0} .
$$

Find the value of $\lambda$ that minimizes the CMF energy $\sqrt{s}$ and establish the relation

$$
\sqrt{s}=E_{\pi}^{0}+\sqrt{m_{X}^{2}+k_{\pi}^{0^{2}}}
$$

where $m_{X}=m_{\mathrm{p}}+m_{\mathrm{n}}$. Derive eventually the maximal pion energy

$$
E_{\pi}^{0}=\frac{s+m_{\pi}^{2}-m_{X}^{2}}{2 \sqrt{s}}
$$

and check that it increases with $\sqrt{s}$ as announced at the beginning.

In the galactic frame, the differential cross section for pion production can be expressed as

$$
\frac{d \sigma}{d E_{\pi}}\left(E_{\mathrm{p}} \rightarrow E_{\pi}\right)=2 \pi p_{\pi} \int_{\cos \alpha}^{1} d \cos \theta \times\left\{E_{\pi} \frac{d^{3} \sigma}{d^{3} p_{\pi}}\right\}_{\mathrm{LI}} .
$$

The angle $\theta$ between the pion momentum $\mathbf{p}_{\pi}$ and the beam axis is related to the pion Lorentz factor in the CMS

$$
\gamma_{\pi}^{\star}=\gamma_{c} \gamma_{\pi}\left(1-\beta_{c} \beta_{\pi} \cos \theta\right) .
$$

The Lorentz factor $\gamma_{c}$ and velocity $\beta_{c}$ of the CMF with respect to the galactic frame have been defined above. They depend on the energy $E_{\mathrm{p}}$ of the impinging proton. As the angle $\theta$ increases, so does $\gamma_{\pi}^{\star}$ until it reaches the maximal value of $\gamma_{\pi}^{0}=E_{\pi}^{0} / m_{\pi}$. The corresponding angle in the galactic system is

$$
\cos \alpha=\frac{1}{\beta_{c} \beta_{\pi}}\left\{1-\left(\frac{\gamma_{\pi}^{0}}{\gamma_{c} \gamma_{\pi}}\right)\right\} .
$$

Whenever $\cos \alpha$ exceeds +1 , the integral (2.91) vanishes. On the contrary, the integration range extends from $\theta=0$ up to $\theta=\pi$ if $\cos \alpha$ turns out to be smaller than -1 . The scaling model leads 
eventually to the positron production differential cross section

$$
\frac{d \sigma}{d E_{e}}\left(E_{\mathrm{p}} \rightarrow \pi^{+}+X \rightarrow E_{e}\right)=\int \frac{d \sigma}{d E_{\pi}}\left(E_{\mathrm{p}} \rightarrow E_{\pi}\right) \times d E_{\pi} \times \mathscr{P}_{+}\left(E_{\pi} \rightarrow E_{e}\right),
$$

where the convolution of the pion production differential cross section with the pion to positron conversion function $\mathscr{P}_{+}\left(E_{\pi} \rightarrow E_{e}\right)$ is performed over pion energy.

(d) Positrons may also be produced through kaons generated in proton-proton collisions. The two main kaon decay modes contribute together a few percent to the total positron production differential cross section. In the first chain of reactions

$$
\begin{aligned}
\mathrm{p}+\mathrm{H} \longrightarrow \mathrm{X}+K^{ \pm} & \\
K^{ \pm} \longrightarrow v_{\mu}+\mu^{ \pm} & \\
& \mu^{ \pm} \longrightarrow v_{\mu}+v_{e}+e^{ \pm},
\end{aligned}
$$

the kaon plays the same role as the pion in the set of decays (2.60). The corresponding cross section is given by relation (2.94) where the pion mass $m_{\pi}$ has been replaced by the kaon mass $m_{K}$. The branching ratio of the $K^{ \pm} \rightarrow v_{\mu}+\mu^{ \pm}$decay channel is $63.5 \%$. The kaon may also decay into a pair of pions, hence the series of reactions

$$
\begin{aligned}
\mathrm{p}+\mathrm{H} \longrightarrow \mathrm{X}+K^{ \pm} \\
K^{ \pm} \longrightarrow \pi^{0}+\pi^{ \pm} \\
\pi^{ \pm} \longrightarrow v_{\mu}+\mu^{ \pm} \\
\mu^{ \pm} \longrightarrow v_{\mu}+v_{e}+e^{ \pm} .
\end{aligned}
$$

The branching ratio of the two pion decay mode is $21 \%$.

Exercise $\mathbf{n}^{0}$ 2-m - Level [1] : Show that the positron production differential cross section is given now by the same convolution as relation (2.94)

$$
\frac{d \sigma}{d E_{e}}\left(E_{\mathrm{p}} \rightarrow K^{+} \rightarrow \pi^{+} \rightarrow E_{e}\right)=\int \frac{d \sigma}{d E_{\pi}}\left(E_{\mathrm{p}} \rightarrow K^{+} \rightarrow E_{\pi}\right) \times d E_{\pi} \times \mathscr{P}_{+}\left(E_{\pi} \rightarrow E_{e}\right),
$$

where the production of pions through the two pion decay channel can be expressed as

$$
\frac{d \sigma}{d E_{\pi}}\left(E_{\mathrm{p}} \rightarrow K^{+} \rightarrow E_{\pi}\right)=\int \frac{d \sigma}{d E_{K}}\left(E_{\mathrm{p}} \rightarrow E_{K}\right) \times d E_{K} \times \frac{\Theta\left(\gamma_{\pi, K}^{-} \leq \gamma_{\pi} \leq \gamma_{\pi, K}^{+}\right)}{2 m_{\pi} \beta_{K} \gamma_{K} \beta_{\pi}^{\prime} \gamma_{\pi}^{\prime}} .
$$

Derive the Lorentz factor of the pion in the kaon rest frame

$$
\gamma_{\pi}^{\prime}=\frac{m_{K}^{2}+m_{\pi^{ \pm}}^{2}-m_{\pi^{0}}^{2}}{2 m_{K} m_{\pi^{ \pm}}}
$$

The Lorentz factor and speed of the kaon in the galactic frame are respectively denoted by $\gamma_{K}=E_{K} / m_{K}$ and $\beta_{K}$. Explain why the Lorentz factor of the pion in the galactic frame is bounded by

$$
\gamma_{\pi, K}^{ \pm}=\gamma_{K} \gamma_{\pi}^{\prime}\left(1 \pm \beta_{K} \beta_{\pi}^{\prime}\right)
$$


Parameterizations of kaon production in proton-proton interactions can be found in [91, 92] in the scaling regime.

(e) Notice finally that useful parametric expressions for the yield and spectra of the stable secondary species produced in $\mathrm{p}-\mathrm{p}$ collisions have been derived from experimental data and summarized in [93].

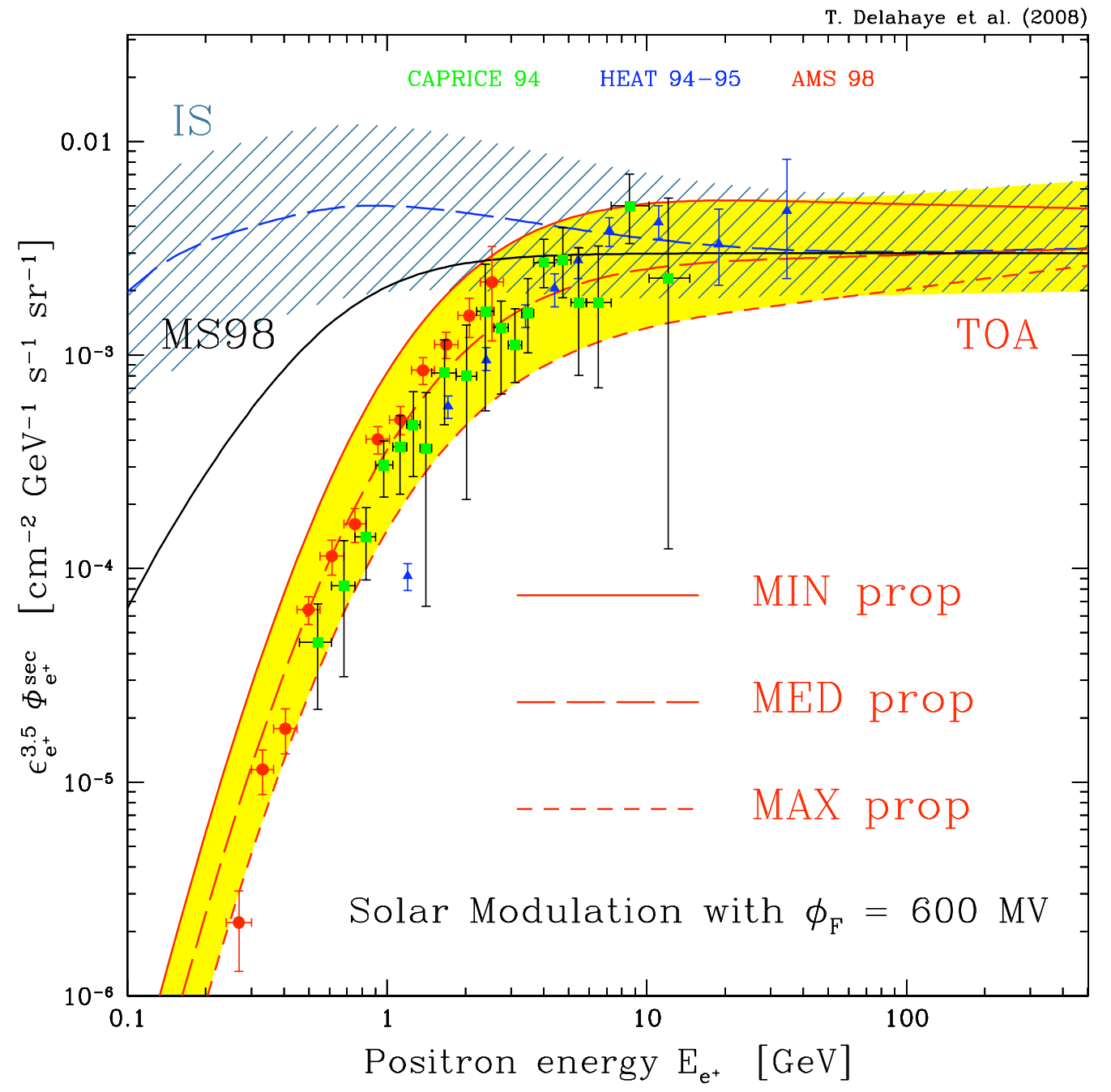

Figure 24: The secondary positron background $\varepsilon_{e^{+}}^{3.5} \times \Phi_{e^{+}}^{\text {sec }}$ is plotted as a function of the positron energy $E_{e^{+}}$. The blue long-dashed curve corresponds to the interstellar (IS) flux derived with a differential production cross section borrowed from [93] and the most recent measurements by BESS [94] of the cosmic ray proton and helium fluxes. Once modulated with a Fisk potential $\phi_{\mathrm{F}}$ of $600 \mathrm{MV}$, it yields the red long-dashed top of the atmosphere (TOA) flux. The blue hatched region is the envelope of the IS positron spectra computed with the set of $\sim 1,600$ different models found in [50] to pass the $\mathrm{B} / \mathrm{C}$ test. This band comprises the theoretical uncertainty associated to cosmic ray propagation. Its TOA counterpart is the yellow strip. The observations by CAPRICE [95] (green squares), HEAT [96] (blue triangles) and AMS [60] (red dots) are also indicated. They lie within the yellow region. The agreement between these measurements and the calculations presented in this section is actually quite good. The black solid line features Moskalenko and Strong's results [89] for their model 08-005 without reacceleration as parameterized by Baltz and Edsjö [86]. 
Cosmic ray protons with energy $E_{\mathrm{p}}$ induce a production of positrons per hydrogen atom whose rate is given by

$$
d \Gamma_{e^{+}}^{\mathrm{sec}}\left(E_{e}\right)=\frac{d \sigma}{d E_{e}}\left(E_{\mathrm{p}} \rightarrow E_{e}\right) \times \beta_{\mathrm{p}} \times\left\{d n_{\mathrm{p}} \equiv \psi_{\mathrm{p}}\left(E_{\mathrm{p}}\right) \times d E_{\mathrm{p}}\right\} .
$$

This leads to the positron production rate per unit of volume and energy

$$
q_{e^{+}}^{\mathrm{sec}}\left(\mathbf{x}, E_{e}\right)=4 \pi n_{H}(\mathbf{x}) \int \Phi_{\mathrm{p}}\left(\mathbf{x}, E_{\mathrm{p}}\right) \times d E_{\mathrm{p}} \times \frac{d \sigma}{d E_{e}}\left(E_{\mathrm{p}} \rightarrow E_{e}\right) .
$$

These relations can be generalized in order to incorporate cosmic ray helium nuclei as well as interstellar helium. The gas of the galactic disc is generally assumed to be homogeneously spread with average hydrogen $n_{\mathrm{H}}$ and helium $n_{\mathrm{He}}$ densities of 0.9 and $0.1 \mathrm{~cm}^{-3}$. The positron production rate $q_{e^{+}}^{\mathrm{sec}}\left(\mathbf{x}, E_{e}\right)$ can be eventually folded in relation (2.44) to yield the positron energy density $\psi_{e^{+}}^{\mathrm{sec}}\left(E_{e}\right)$ and the corresponding flux $\Phi_{e^{+}}^{\mathrm{sec}}\left(E_{e}\right)$ at the Earth.

Exercise $\mathbf{n}^{0}$ 2-n - Level [1] : The cosmic ray proton $\Phi_{\mathrm{p}} \equiv \beta_{\mathrm{p}} \psi_{\mathrm{p}} / 4 \pi$ and helium $\Phi_{\alpha}$ fluxes depend a priori on the position $\mathbf{x}_{S}$ of the source. Secondary positrons are produced inside the galactic disc. Those detected at the Earth originate mostly from the solar neighborhood. Thus we can safely disregard the radial dependence of $\Phi_{\mathrm{p}}$ and $\Phi_{\alpha}$ in the calculation of the positron source term and take the solar values which can be found for instance in $[51,94]$. The secondary positron source term for proton-hydrogen collisions becomes

$$
q_{e^{+}}^{\mathrm{sec}}\left(\odot, E_{e}\right)=4 \pi n_{H} \int \Phi_{\mathrm{p}}\left(\odot, E_{\mathrm{p}}\right) \times d E_{\mathrm{p}} \times \frac{d \sigma}{d E_{e}}\left(E_{\mathrm{p}} \rightarrow E_{e}\right) .
$$

Use this approximation into relation (2.44) to derive the secondary positron flux

$$
\Phi_{e^{+}}^{\mathrm{sec}}\left(\odot, \varepsilon \equiv E_{e} / E_{0}\right)=\frac{\beta_{e^{+}}}{4 \pi} \times \frac{\tau_{E}}{\varepsilon^{2}} \times \int_{\varepsilon}^{+\infty} d \varepsilon_{S} \times \tilde{I}\left(\lambda_{\mathrm{D}}\right) \times q_{e^{+}}^{\mathrm{sec}}\left(\odot, \varepsilon_{S}\right) .
$$

The integral $\tilde{I}$ is the convolution of the reduced positron Green function over the galactic disc alone

$$
\tilde{I}\left(\lambda_{\mathrm{D}}\right)=\int_{\text {disc }} d^{3} \mathbf{x}_{S} \tilde{G}\left(\mathbf{x}_{\odot} \leftarrow \mathbf{x}_{S} ; \lambda_{\mathrm{D}}\right)
$$

The method outlined in this exercise has been used to derive the positron flux featured in figure 24 . At $1 \mathrm{GeV}$, the width of the IS uncertainty strip corresponds to an increase by a factor of $\sim 6$ between the smallest and the largest positron fluxes allowed by the $\mathrm{B} / \mathrm{C}$ constraint. That factor decreases down to 3.9 at $10 \mathrm{GeV}$ and reaches 2.9 at $100 \mathrm{GeV}$. Once modulated with a Fisk potential $\phi_{\mathrm{F}}$ of $600 \mathrm{MV}$, the blue hatched region is transformed into the yellow TOA band. The latter is delineated by the MAX and MIN configurations of table 4. Quite surprisingly, the MIN model (red solid curve) corresponds now to the maximal secondary positron flux whereas the MAX configuration (red short-dashed line) yields the minimal prediction. According to relation (2.104), the positron flux is given by the convolution over the energy $\varepsilon_{S}$ of the source term $q_{e^{+}}^{\text {sec }}\left(\varepsilon_{S}\right)$ with the halo integral $\tilde{I}\left(\lambda_{\mathrm{D}}\right)$. As the energy $\varepsilon_{S}$ increases from $\varepsilon$ to infinity, the positron diffusion length $\lambda_{\mathrm{D}}-$ which depends on both $\varepsilon$ and $\varepsilon_{S}$-increases. As long as $\lambda_{\mathrm{D}}$ remains smaller than the disc half-thickness $h$ of $100 \mathrm{pc}$, the halo integral $\tilde{I}$ is equal to 1 . However, it drops rapidly below that value and 
vanishes as soon as $\lambda_{\mathrm{D}}$ exceeds $h$. The diffusion length $\lambda_{\mathrm{D}}$ is proportional to $\left(K_{0} \tilde{\tau}\right)^{1 / 2}$ so that its increase with $\varepsilon_{S}$ - keeping $\varepsilon$ fixed - is dominated by the diffusion coefficient $K_{0}$. Hence the smaller $K_{0}$, the smaller $\lambda_{\mathrm{D}}$ and consequently the more the halo integral $\tilde{I}\left(\lambda_{\mathrm{D}}\right)$ contributes to the positron flux (2.104). The decrease of the DH half-thickness $L$ from the MAX to the MIN models has the opposite effect but cannot prevent the MIN configuration from providing the largest secondary positron flux. The various positron measurements fall nicely inside the TOA yellow band. Our modeling of cosmic ray propagation supplemented by Baltz \& Edsjö's treatment [86] of energy losses is therefore well supported by observation. The careful reader could nevertheless notice a small deficiency of the data below a few $\mathrm{GeV}$ associated to a possible excess above.

This trend is mildly indicated by the HEAT data [97] which seem to point toward a possible excess of the positron fraction

$$
\frac{e^{+}}{e^{+}+e^{-}} \equiv \frac{\Phi_{e^{+}}^{\text {tot }}}{\Phi_{e^{+}}^{\text {tot }}+\Phi_{e^{-}}^{\text {tot }}}
$$

for energies above $10 \mathrm{GeV}$ and with respect to the calculations for the secondary component [89] available when that analysis was performed. Different astrophysical contributions to the positron fraction in the $10 \mathrm{GeV}$ region have been explored [97], but only more accurate and energy extended data could shed light on the effective presence of a bump in the positron fraction and on its physical interpretation. Alternatively, it has been conjectured that the possible excess of positrons found in the HEAT data could be due to the presence of DM annihilation in the galactic halo [86, 98]. This interpretation, though very exciting, is at some point limited by the uncertainties in the halo structure and in the cosmic ray propagation modeling. Equations (2.8), (2.44) and (2.59) can be combined to yield the positron flux generated by the WIMP annihilations taking place within the Milky Way DH

$$
\Phi_{e^{+}}^{\mathrm{DM}}\left(\odot, \varepsilon \equiv E_{e} / E_{0}\right)=\mathscr{F} \frac{\tau_{E}}{\varepsilon^{2}} \times \int_{\varepsilon}^{m_{\chi} / E_{0}} d \varepsilon_{S} f\left(\varepsilon_{S}\right) \tilde{I}_{\mathrm{DM}}\left(\lambda_{\mathrm{D}}\right)
$$

The halo integral $\tilde{I}_{\mathrm{DM}}$ is the convolution of the reduced positron propagator $\tilde{G}$ with the square of the DM galactic density

$$
\tilde{I}_{\mathrm{DM}}\left(\lambda_{\mathrm{D}}\right)=\int_{\mathrm{DH}} d^{3} \mathbf{x}_{S} \tilde{G}\left(\mathbf{x}_{\odot} \leftarrow \mathbf{x}_{S} ; \lambda_{\mathrm{D}}\right)\left\{\frac{\rho\left(\mathbf{x}_{S}\right)}{\rho_{\odot}}\right\}^{2} .
$$

The latter has already been given in relation (2.34) and table 6 where extremely different situations have been grouped together. In particular, the DM density in the solar neighborhood is subject to large variations between the various models. Following the analysis by [88], we will consider instead DM profiles normalized to a fixed solar value $\rho_{\odot}$ of $0.3 \mathrm{GeV} \mathrm{cm}^{-3}$. These are generically given by

$$
\rho(r)=\rho_{\odot}\left(\frac{r_{\odot}}{r}\right)^{\gamma}\left\{\frac{1+\left(r_{\odot} / r_{\mathrm{s}}\right)^{\alpha}}{1+\left(r / r_{\mathrm{s}}\right)^{\alpha}}\right\}^{(\beta-\gamma) / \alpha},
$$

where $r_{\odot}=8.5 \mathrm{kpc}$ is the galactocentric distance of the solar system. Notice that $r$ denotes here once again the radius in spherical coordinates. Two profiles are featured in figure 25 and the corresponding parameters are summarized in table 7. 


\begin{tabular}{|l||c|c|c||c|}
\hline Halo model & $\alpha$ & $\beta$ & $\gamma$ & $r_{\mathrm{s}}[\mathrm{kpc}]$ \\
\hline \hline Cored isothermal [2] & 2 & 2 & 0 & 5 \\
NFW 97 [79] & 1 & 3 & 1 & 20 \\
\hline
\end{tabular}

Table 7: Parameters in equation (2.109) for the DM halo models considered in figure 25. Values are similar to those listed in table 6 . The solar neighborhood DM density $\rho_{\odot}$ is now set equal to the canonical value of $0.3 \mathrm{GeV} \mathrm{cm}{ }^{-3}$ for both the isothermal sphere and the NFW profile.

As shown in figure 25, the half-thickness $L$ of the diffusive halo has a direct influence on the overall shape of $\tilde{I}_{\mathrm{DM}}$ as a function of $\lambda_{\mathrm{D}}$. In the left panel, an isothermal distribution has been assumed whereas the right panel features the case of a NFW profile. For small values of $L$ - see the green
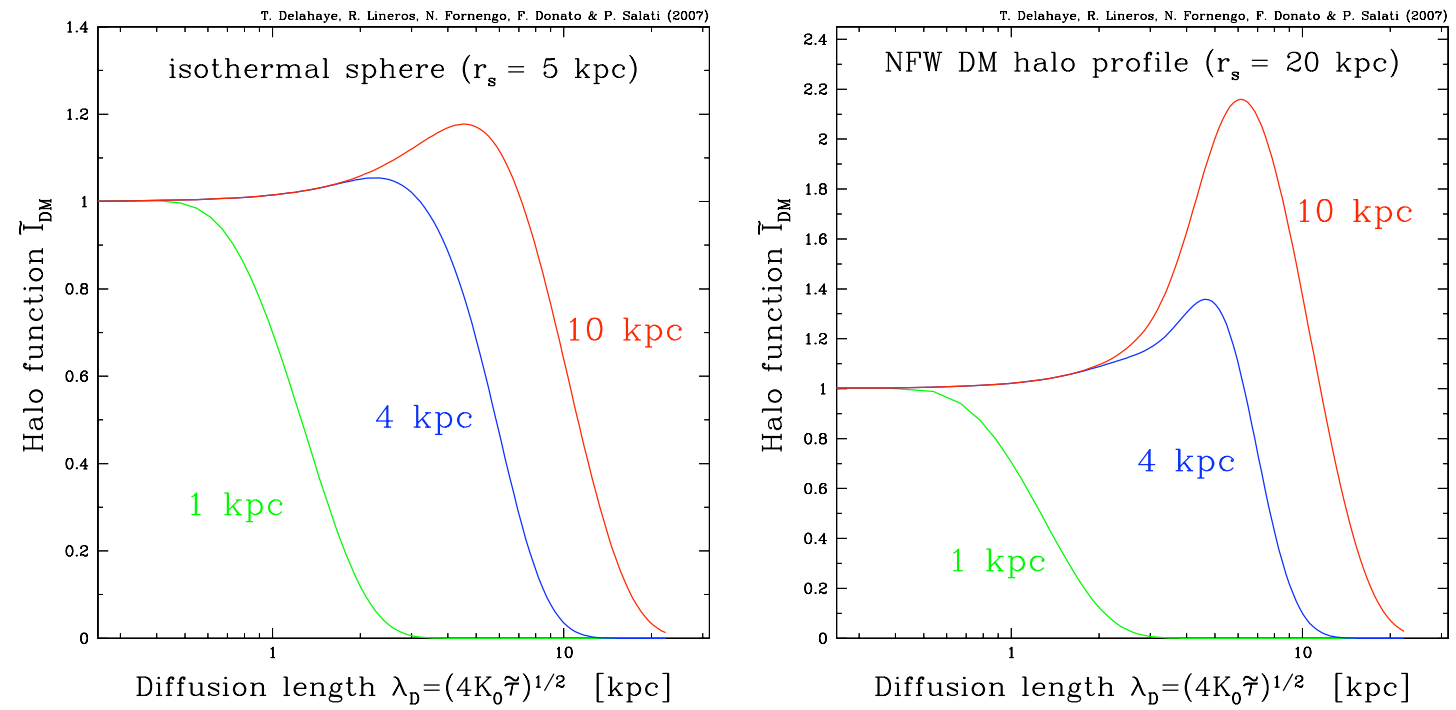

Figure 25: The halo convolution $\tilde{I}_{\mathrm{DM}}$ is presented as a function of the positron diffusion length $\lambda_{\mathrm{D}}$ for various values of the DH half-thickness $L$. The left panel features the case of an isothermal DM distribution whereas a NFW profile has been assumed in the right panel - see table 7. When $L$ is large enough for the positron horizon to reach the galactic center and its denser DM distribution, a maximum appears in the curves for $\lambda_{\mathrm{D}} \sim r_{\odot}$. Figures from [88].

curve for which $L=1 \mathrm{kpc}$ - the positron horizon is fairly limited. Because the positrons detected at the Earth merely originate from a very near region, the DM profile which is probed is essentially uniform. The DH integral $\tilde{I}_{\mathrm{DM}}$ is unity below $\lambda_{\mathrm{D}} \sim L$ and collapses for larger values of the diffusion length. For a thicker slab, the cosmic ray positron flux at the Earth gets sensitive to the center of the Galaxy. That is why the halo integral $\tilde{I}_{\mathrm{DM}}$ exhibits a maximum for a diffusion length $\sim 5-7$ $\mathrm{kpc}$, a value close to the galactocentric distance $r_{\odot}=8.5 \mathrm{kpc}$ of the solar system. In both panels, the larger $L$, the more visible the bump. Notice also that the steeper the DM profile, the higher the maximum. 


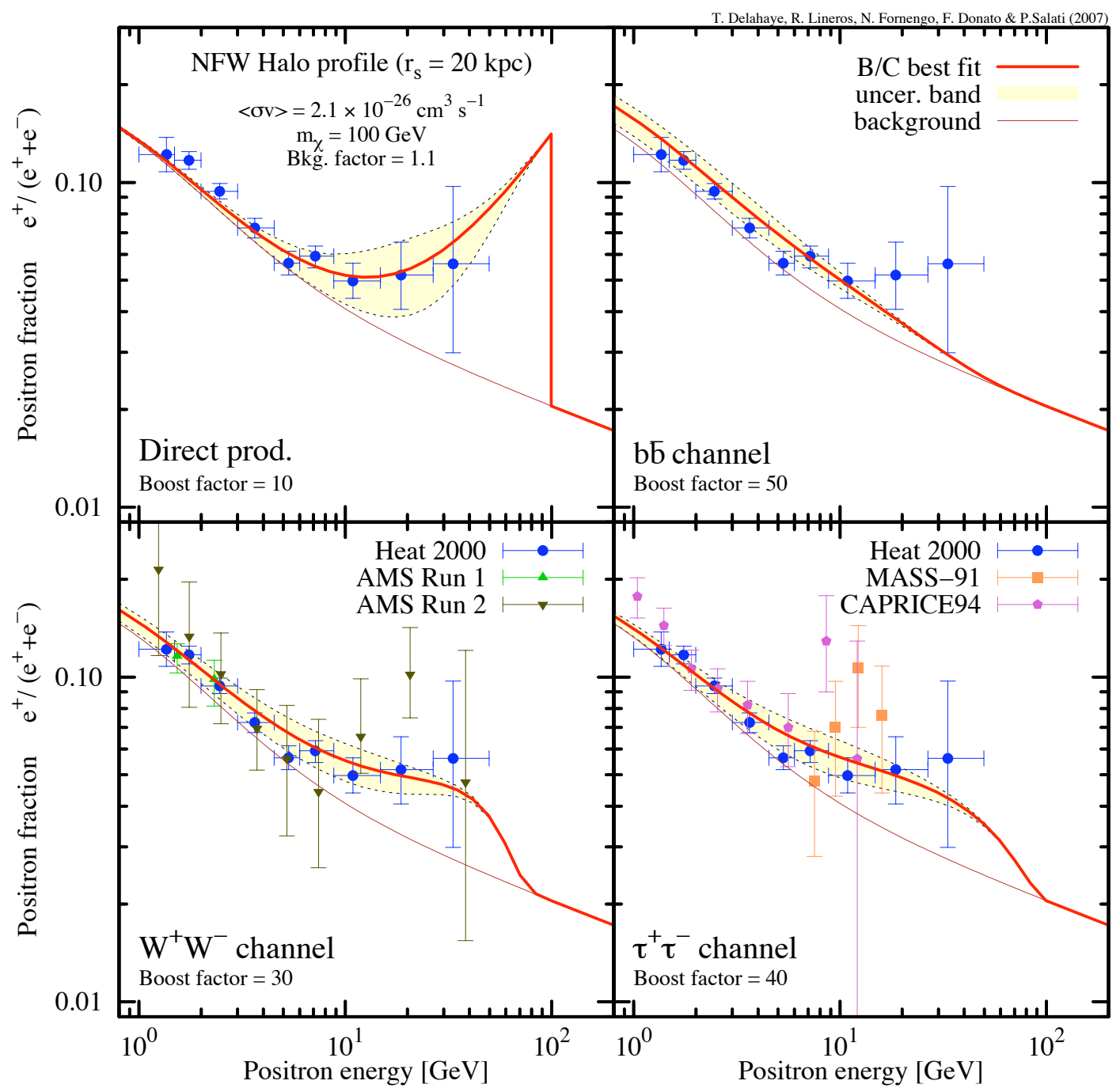

Figure 26: The positron fraction $e^{+} /\left(e^{-}+e^{+}\right)$is plotted versus the positron energy $E$ for a $100 \mathrm{GeV}$ WIMP in the case of the NFW profile of table 7. The four panels refer to different annihilation final states : direct $e^{+} e^{-}$production (top left), $b \bar{b}$ (top right), $W^{+} W^{-}$(bottom left) and $\tau^{+} \tau^{-}$(bottom right). In each panel, the brown thin solid line stands for the positron background borrowed from [89] and parameterized by [86]. The red thick solid curve refers to the total positron flux where the signal is propagated with the best-fit choice of the astrophysical parameters - the configuration MED of table 4. The yellow area features the total uncertainty band arising from cosmic ray propagation. The different models found in [50] to be compatible with the $\mathrm{B} / \mathrm{C}$ ratio all yield a positron fraction which is enclosed inside this yellow strip. Experimental data from HEAT [97], AMS [99, 100], CAPRICE [95] and MASS [101] are also presented for comparison. Figure from [88].

In figure 26, the positron fraction (2.106) is presented as a function of the positron energy $E$. The total positron flux at the Earth

$$
\Phi_{e^{+}}^{\mathrm{tot}}=\Phi_{e^{+}}^{\mathrm{DM}}+\Phi_{e^{+}}^{\mathrm{sec}}
$$

encompasses the annihilation signal and a background component for which the results of [89] 
as parameterized by [86] have been used - see the brown thin solid lines. The mass of the DM species is $100 \mathrm{GeV}$ and a NFW profile has been assumed. The observations featured in the various panels are indications of a possible excess of the positron fraction for energies above $10 \mathrm{GeV}$. Those measurements may be compared to the red thick solid curves that correspond to the MED configuration of table 4. In order to get a reasonable agreement between the DM predictions and the data, the annihilation signal has been boosted by an energy-independent factor ranging from 10 to 50 as indicated in each panel. At the same time, the positron background has been shifted upward from its reference value [86] by a small amount of 10\%. As is clear in the upper left panel, the case of direct production offers a very good agreement with the potential HEAT excess. Notice how well all the data points lie within the yellow uncertainty band. A boost factor of 10 is enough to obtain an excellent agreement between the measurements and the median flux. A smaller value would be required for a flux at the upper envelope of the uncertainty strip. The $W^{+} W^{-}$and $\tau^{+} \tau^{-}$ channels may also reproduce reasonably well the observations, especially once the uncertainty arising from cosmic ray propagation throughout the Milky Way diffusive halo is taken into account. They need though larger boost factors of the order of 30 to 40 . On the contrary, softer production channels, like the $b \bar{b}$ case, are unable to match the features of the putative HEAT excess. For all annihilation channels, the uncertainty strips get thinner at high energies. The typical positron diffusion length $\lambda_{\mathrm{D}}$ decreases in this regime and the halo integral $\tilde{I}_{\mathrm{DM}}$ probes mostly the solar environment with a value of the DM density approximately given by $\rho_{\odot}$. With a value of $\tilde{I}_{\mathrm{DM}}$ close to 1 in relation (2.107), the primary positron flux $\Phi_{e^{+}}^{\mathrm{DM}}$ is subject to little variations as the set of models compatible with $\mathrm{B} / \mathrm{C}$ is scanned. The uncertainty areas surprisingly tend to shrink also at low energies, a regime where the positron horizon is the furthest and where the details of galactic propagation are expected to be the most important. Actually, the annihilation signal $\Phi_{e^{+}}^{\mathrm{PM}}$ turns out to be completely swamped in the positron background $\Phi_{e^{+}}^{\mathrm{sec}}$. In particular, the signal from direct production stands up over the background only for energies larger than $5 \mathrm{GeV}$. The corresponding uncertainty on the positron fraction is at most of the order of $50 \%$ for energies between 10 and $20 \mathrm{GeV}$. In the other cases, the uncertainty bands are even thinner. Beware finally of the positron background which also suffers from uncertainties due to secondary production processes and propagation. The latter effect is presented in figure 24 but its joint influence on both the primary and secondary fluxes has not yet been investigated as I am completing this proceedings.

\subsection{High-energy photons and the galactic center}

WIMP annihilations also generate high-energy photons whose energy distribution is described by the function $f\left(E_{\gamma}\right) \equiv d N_{\gamma} / d E_{\gamma}$. The corresponding flux at the Earth - from the direction toward which the unit vector $\mathbf{u}$ is pointing - is given by the product

$$
\Phi_{\gamma}^{\mathrm{DM}}\left(E_{\gamma}, \mathbf{u}\right)=\frac{\eta}{4 \pi}\left\{\frac{\left\langle\sigma_{\mathrm{ann}} v\right\rangle f\left(E_{\gamma}\right)}{m_{\chi}^{2}}\right\} \times \int_{\operatorname{los}} \rho^{2}(\mathbf{x}) d s .
$$

This formula is often seen as the emblem of particle astrophysics insofar as it exhibits two distinct pieces. The first part is related to particle physics and encodes informations on the WIMP properties such as its mass and annihilation cross section. The second term is clearly astrophysical in nature and deals with the distribution of DM along the line of sight (los) toward which $\mathbf{u}$ is pointing. 
Let us first concentrate on the particle physics aspect and in particular on the gamma ray distribution generated by WIMP annihilations. Three different contributions to $f\left(E_{\gamma}\right)$ need to be considered.

(i) The dominant source of high-energy photons is related to the production of quarks and gauge bosons which subsequently fragmentate and decay into secondary gamma rays through essentially the two-photon decays of neutral pions

$$
\chi+\chi \rightarrow q \bar{q}, W^{+} W^{-}, \ldots \rightarrow \gamma+\ldots .
$$

For each annihilation channel, this leads to a continuum whose spectrum has been parametrized in [83] with the generic form

$$
\frac{d N_{\gamma}^{\text {cont }}}{d x}=x^{-1.5} \exp \left(a+b x+c x^{2}+d x^{3}\right)
$$

where $x=E_{\gamma} / m_{\chi}$. This distribution exhibits a characteristic $x^{-1.5}$ power law behaviour for small values of $x$ and a smooth cutoff when the photon energy is close to the WIMP mass.

(ii) A particularly clear signal of the presence of DM species inside the Milky Way halo is the production of monochromatic gamma rays through the reaction

$$
\chi+\chi \rightarrow \gamma+\gamma \& \gamma+Z^{0} .
$$

This process gives rise to characteristic line signals which cannot be mistaken for some conventional astrophysical source and which would unequivocally signal the presence of an exotic component inside the Galaxy should a peak be detected in the high-energy spectrum.

Exercise $\mathbf{n}^{0}$ 2-o - Level [1] : Show that the energy of the photons produced in reaction (2.114) is equal to $E_{\gamma}=m_{\chi}$ and

$$
E_{\gamma}=m_{\chi}-\frac{m_{Z}^{2}}{4 m_{\chi}}
$$

However, WIMPs have to be electrically neutral and the production of monochromatic photons is necessarily mediated by loop diagrams. It is generally suppressed and the integrated photon yield amounts to $\sim 10^{-3}$ of the total. This leads to a clear but faint signal which is beyond the reach of current detectors unless the process is efficiently enhanced as in the case of heavy Wino-like neutralinos - these DM candidates are highly degenerate in mass with charginos [102, 67].

(iii) Finally, as already pointed out in [103], a single photon may be produced through internal bremsstrahlung as a WIMP pair annihilates. The gamma ray is radiated by the charged particles that are either exchanged or produced. This process becomes particularly important for a sizable branching ratio into electron-positron pairs as in the case of $\mathrm{MeV}$ dark matter [104] or KaluzaKlein inspired models [105]. The final state radiation spectrum associated to the production of the charged lepton pair $l^{+} l^{-}$is, in leading logarithmic order, well approximated by $[105,106]$

$$
\frac{d N_{\gamma}^{\mathrm{IB}}}{d x}=\frac{d\left(\sigma_{l^{+} l^{-} \gamma} v\right) / d x}{\sigma_{l^{+} l^{-}} v} \simeq \frac{\alpha_{\mathrm{em}}}{\pi} \frac{\left(x^{2}-2 x+2\right)}{x} \ln \left\{\frac{m_{\chi}^{2}}{m_{l}^{2}}(1-x)\right\} .
$$


Internal bremsstrahlung produces more photons than fragmentation does near the upper edge $E_{\gamma}=m_{\chi}$ of the spectrum and is thus responsible for a characteristic sharp cutoff there. For Wino-like heavy DM species, photons are radiated by final $W^{+} W^{-}$pairs as discussed in [74].

Exercise $\mathbf{n}^{0} \mathbf{2}-\mathbf{p}$ - Level [2] : Derive relation (2.111) and show that the gamma ray flux is given by

$$
\Phi_{\gamma}^{\mathrm{DM}}\left(E_{\gamma}=m_{\chi}, \mathbf{u}\right)=1.88 \times 10^{-13} \text { photons } \mathrm{cm}^{-2} \mathrm{~s}^{-1} \mathrm{sr}^{-1} \times \frac{\left\langle\sigma_{\gamma \gamma} \nu\right\rangle_{29}}{m_{100}{ }^{2}} \times J(\mathbf{u})
$$

in the case of the two-photon line. The annihilation cross section $\left\langle\sigma_{\gamma \gamma} v\right\rangle$ and the neutralino mass $m_{\chi}$ are respectively expressed in units of $10^{-29} \mathrm{~cm}^{3} \mathrm{~s}^{-1}$ and $100 \mathrm{GeV}$. A Majorana type DM species has been assumed here with $\eta \equiv 1 / 2$. The line of sight integral $J(\mathbf{u})$ in the direction toward which the unit vector $\mathbf{u}$ is pointing has been defined by

$$
J(\mathbf{u})=\left\{\rho_{\odot}^{2} r_{\odot}\right\}^{-1} \times \int_{\operatorname{los}} \rho^{2}(\mathbf{x}) d s,
$$

with a solar neighborhood $D M$ density of $\rho_{\odot}=0.3 \mathrm{GeV} \mathrm{cm}^{-3}$. The galactocentric distance $r_{\odot}$ of the solar system has been set equal to $8.5 \mathrm{kpc}$.

The line of sight integral $J$ depends on the galactic DM distribution. The various halo models of table 6 yield similar values all over the sky except in the direction of the galactic center where predictions can vary by several orders of magnitude depending on the assumed profile. As shown in [107] - where it has been averaged over a solid angle of $10^{-5} \mathrm{sr}$ - the line of sight integral is respectively equal to 30 and $1.45 \times 10^{4}$ for the two models of table 7 . In the case of a Moore profile that has been adiabatically compressed by the collapse of the supermassive black hole lying at the center of the Galaxy - see section 3.2 and figure 32 for a discussion of that effect $-J$ may even be as large as $3 \times 10^{8}$. The astrophysical uncertainties are enormous of course. The possibility of a strong signal has triggered a febrile activity around the galactic center and motivated observations focusing in that direction. Let us concentrate for instance on the NFW profile of table 7. For small galactocentric distances, the DM distribution simplifies into

$$
\rho(r)=\aleph \rho_{\odot}\left\{\frac{r_{\odot}}{r}\right\} .
$$

where the normalization constant $\aleph$ is found equal to 2.03 .

Exercise $\mathbf{n}^{0} \mathbf{2 - q}-$ Level [1] : A line of sight is separated from the galactic center by the angle $\alpha$. Assume that $\alpha$ is small and show that the integral $J$ simplifies into

$$
J_{N F W}(\alpha)=\aleph^{2} \frac{\pi}{\alpha} .
$$

Compute then the average value of the line of sight integral $J$ over a disc whose angular radius is $\theta$ and establish that

$$
\langle J\rangle_{N F W}(\theta) \equiv \frac{1}{\pi \theta^{2}} \int_{0}^{\theta} 2 \pi \alpha J_{N F W}(\alpha) d \alpha=\aleph^{2} \frac{2 \pi}{\theta} .
$$

Check that this leads numerically to $\langle J\rangle_{N F W}(\theta)=1484 \times\left(1^{\circ} / \theta\right)$. 
High-energy photons can be detected by satellite borne instruments orbiting the Earth and therefore free from the screening of its atmosphere. To quote just a few experiments, let us mention the Compton Gamma Ray Observatory (CGRO) with its EGRET instrument devoted to the search for GeV photons [108] as well as the recently launched GLAST satellite [109] and the forthcoming AMS-02 mission on board the international space station [110]. Because of the reduced payload which can be carried up in orbit, the collecting area of these instruments is not very large and statistics are reduced even for a mission of several years. Above a few dozens of GeV, atmospheric Cherenkov telescopes (ACT) come into play and offer a nice alternative which complements space observations. The effective detecting area can be actually quite large insofar as a high-energy gamma ray impinging upon the upper atmosphere generates a shower and is degraded into many optical photons which ordinary telescopes spread on the ground can detect. The track left in the focal planes of the latter gives the direction of the shower on the sky so that several telescopes allow the stereoscopic reconstruction of the direction of the initial particle. The Cherenkov light illuminates on the ground a disc whose diameter is of order $250 \mathrm{~m}$ hence a large effective area of $\sim 0.05 \mathrm{~km}^{2}$ for each telescope, to be compared with the typical square-meter collecting surface of a space instrument. The intensity of the image depends on the energy of the incident photon. The ACT technique is thus a powerful tool allowing the observation of the high-energy gamma ray sky. However, as for any other observation, a gamma ray signal is only detectable as long as it emerges above the background. More specifically, the signal has to be larger than the statistical fluctuations - also called the noise - of the latter. For atmospheric Cherenkov telescopes, the dominant background arises from cosmic ray electrons that penetrate the atmosphere inside which they produce electromagnetic showers of the same type as those induced by high-energy photons. It is not possible to distinguish photons from electrons since both species lead to the same light pattern on the ground. The cosmic ray electron flux is given by [111]

$$
\Phi_{e^{-}}\left(E_{e}\right)=6.4 \times 10^{-2} \mathrm{GeV}^{-1} \mathrm{~cm}^{-2} \mathrm{~s}^{-1} \mathrm{sr}^{-1}\left(E_{e} / 1 \mathrm{GeV}\right)^{-3.3 \pm 0.2} .
$$

Impinging cosmic ray hadrons also interact with the atmosphere. The showers which they generate tend to develop at a lesser altitude and are more widely spread on the ground than those of the electromagnetic type. Stereoscopy is a powerful tool to discriminate hadrons from electrons and gamma rays since the pattern recognition of the light pool is then possible. Observations performed between $50 \mathrm{GeV}$ and $2 \mathrm{TeV}$ yield a hadron flux [112]

$$
\Phi_{\text {had }}(E)=1.8 \mathrm{GeV}^{-1} \mathrm{~cm}^{-2} \mathrm{~s}^{-1} \mathrm{sr}^{-1}(E / 1 \mathrm{GeV})^{-2.75} .
$$

A small fraction $\kappa_{c r}$ of the hadron induced showers are mistaken for gamma ray events though. This is the dominant source of background at high energy as hadrons have a harder spectrum than electrons. The HESS collaboration quotes a rejection factor of one misidentified event over a sample of 300 showers generated by cosmic ray protons. Satellite borne instruments do not suffer from the same flaws. However, point sources can still be buried inside a galactic gamma ray diffuse emission produced above $100 \mathrm{MeV}$ by the spallation of the interstellar gas by cosmic ray nuclei. The flux of this diffuse emission is given by the convolution along the line of sight of the hydrogen density $n_{H}$ with the gamma ray emissivity $I_{H}$ per hydrogen atom

$$
\Phi_{\gamma}^{\mathrm{sec}}\left(E_{\gamma}, \mathbf{u}\right)=\int_{\operatorname{los}} n_{H}(\mathbf{x}) \times I_{H}\left(\mathbf{x}, E_{\gamma}\right) \times d s .
$$


The galactic diffuse emission is therefore made of secondary photons resulting mostly from the interactions of high-energy cosmic ray protons on the hydrogen of the Milky Way disc. This process has already been discussed for antiprotons and positrons. It has been respectively described by the source terms $q_{\overline{\mathrm{p}}}^{\mathrm{sec}}\left(\mathbf{x}, E_{\overline{\mathrm{p}}}\right)$ - see relation (2.15) of section 2.2 - and $q_{e^{+}}^{\mathrm{sec}}\left(\mathbf{x}, E_{e}\right)$ - see relation (2.102) of section 2.3. The photon emissivity per hydrogen atom is defined as

$$
I_{H}\left(\mathbf{x}, E_{\gamma}\right)=\frac{q_{\gamma}^{\sec }\left(\mathbf{x}, E_{\gamma}\right)}{4 \pi n_{H}(\mathbf{x})}
$$

and may be written as the convolution over proton energy of the cosmic ray proton flux with the differential photo-production cross section of proton-proton interactions

$$
I_{H}\left(\mathbf{x}, E_{\gamma}\right)=\int \Phi_{\mathrm{p}}\left(\mathbf{x}, E_{\mathrm{p}}\right) \times d E_{\mathrm{p}} \times \frac{d \sigma}{d E_{\gamma}}\left(E_{\mathrm{p}} \rightarrow E_{\gamma}\right) .
$$

The emissivity $I_{H}$ is expressed in units of $\mathrm{GeV}^{-1} \mathrm{~s}^{-1} \mathrm{sr}^{-1}$ since it is essentially a production rate per unit of energy and solid angle. In the solar neighborhood, the gamma ray emissivity of each hydrogen atom illuminated by the local cosmic ray protons may be approximated by the power law [113]

$$
I_{H}\left(\odot, E_{\gamma}\right)=2 \times 10^{-35} \mathrm{GeV}^{-1} \mathrm{~s}^{-1} \mathrm{sr}^{-1}\left(E_{\gamma} / 1 \mathrm{TeV}\right)^{-2.73} .
$$

This galactic diffuse emission dominates over an extragalactic component which has been measured [114] with the EGRET instrument on board the CGRO satellite

$$
\Phi_{\gamma}^{\mathrm{eg}}\left(E_{\gamma}\right)=7.32 \pm 0.34 \times 10^{-9} \mathrm{MeV}^{-1} \mathrm{~cm}^{-2} \mathrm{~s}^{-1} \mathrm{sr}^{-1}\left(E_{\gamma} / 451 \mathrm{MeV}\right)^{-2.10 \pm 0.03} .
$$

Let us concentrate now on the sensitivity of an atmospheric Cherenkov telescope of the HESS caliber. Such an instrument has an effective detecting area $\mathscr{S}$ of order $0.1 \mathrm{~km}^{2}$ with four mirrors spread on a $300 \mathrm{~m} \times 300 \mathrm{~m}$ square. One of the main targets of HESS is the galactic center where the putative WIMPs might have collapsed, producing a hot spot in the gamma ray sky. We will assume in what follows that the DM profile is given by the NFW distribution of table 7 . The effective time $\mathscr{T}$ during which the observation of the galactic center is performed - disregarding the periods of daylight as well as the nights during which the Moon shines - will be taken to be a month. We infer an approximate acceptance of

$$
\mathscr{S} \times \mathscr{T} \approx 0.01 \mathrm{~km}^{2} \mathrm{yr} .
$$

Let us also assume that our HESS type telescope surveys a circular field of view with angular radius $\theta \sim 1^{\circ}$ surrounding the center of the Milky Way. The self-annihilation of WIMPs into photon pairs is the only process in which we will be interested here. It produces monochromatic gamma rays with energy $E_{\gamma} \equiv m_{\chi}$.

Exercise $\mathbf{n}^{0}$ 2-r - Level [1] : Average the flux $\Phi_{\gamma}^{\text {DM }}$ given by expression (2.117) over the field of view and take into account the acceptance of the instrument in order to derive the total number of line photons collected during the run

$$
N_{\gamma}^{\mathrm{DM}}\left(E_{\gamma} \equiv m_{\chi}\right)=840 \text { photons } \times \frac{\left\langle\sigma_{\gamma \gamma} v\right\rangle_{29}}{m_{100}^{2}} \times\left\{\frac{\theta}{1^{\circ}}\right\} .
$$


These monochromatic gamma rays are detected within some energy bin whose width is set by the resolution of the telescope. We may safely take an energy resolution $\sigma(E) / E$ of order $10 \%$ to be compared to the value of $15 \%$ in the case of HESS. The energy bin that contains the line has thus a width $\Delta E_{\gamma}$ of order $0.2 \times m_{\chi}$. The better the energy resolution, the narrower the line bin and the more visible the peak in the photon spectrum. All the bins are filled up predominantly by misidentified hadron and electron events as discussed above. The monochromatic signal from annihilating DM species is detectable only if it exceeds the statistical fluctuations of that background.

Exercise $\mathbf{n}^{0}$ 2-s - Level [1] : Electron induced showers are assumed here to be the only source of background even though this may not be true at high energy where hadrons come into play. Compute the number of background events collected during the run inside the line bin. The latter is centered on the line energy $E_{\gamma} \equiv m_{\chi}$. Use the cosmic ray electron flux (2.122) to establish that

$$
N_{\gamma}^{\text {back }}=9.7 \times 10^{5} \text { photons } \times\left(m_{100}\right)^{-2.3} \times\left\{\frac{\theta}{1^{\circ}}\right\}^{2} .
$$

The DM line signal $N_{\gamma}^{\mathrm{DM}}$ is deeply swamped into the background $N_{\gamma}^{\text {back }}$ and seems hopelessly out of reach. However, electronic and hadronic events are homogeneously spread on the sky since these cosmic radiations are isotropic at the Earth. Changing the direction towards which the telescope is pointing does not affect the number of background events. On the contrary, the line signal disappears as soon as the field of view no longer encompasses the galactic center. By alternatively pointing the telescope on and off the source makes it possible to detect the line signal provided that it exceeds the statistical background fluctuations. A good estimate for the latter is given by the Poisson noise $\sqrt{N_{\gamma}^{\text {back }}}$. Detection of a signal with a significance of $\mathrm{n}$ is therefore achieved when the signal to noise ratio is equal to

$$
N_{\gamma}^{\mathrm{DM}} / \sqrt{N_{\gamma}^{\mathrm{back}}}=\mathrm{n}
$$

Exercise $\mathbf{n}^{0}$ 2-t - Level [1] : Show that our HESS like telescope would detect a NFW distribution of DM species at the galactic center with a signal to ratio ratio of 3-sigma should their mass and two-photon annihilation cross section fulfill the condition

$$
\left\langle\sigma_{\gamma \gamma} v\right\rangle \geq 3.5 \times 10^{-29} \mathrm{~cm}^{3} \mathrm{~s}^{-1} \times\left(m_{100}\right)^{0.85} .
$$

The HESS collaboration has recently observed the galactic center with unprecedented accuracy $[115,116]$ above $160 \mathrm{GeV}$. A strong point-like source called HESS J1745-290 is detected at the positions of the supermassive black hole Sagittarius $A^{*}$ and the supernova remnant Sagittarius A East. Its spectral index is $\sim 2.25$. An important gamma ray diffuse emission is also seen with a similar spectral index. It is clearly associated with the band of molecular clouds lying in the 
central region and mapped from their CS line. These clouds have been recently penetrated by cosmic ray protons and nuclei accelerated by some nearby supernova event. The correlation between the intensity of the $\mathrm{TeV}$ diffuse emission and the gas column density of the clouds is striking and suggests a uniform density of cosmic rays. No line is observed though. Moreover, the gamma ray spectrum is too hard to be compatible with a WIMP annihilation signal. As shown in [105], a Kaluza-Klein DM species could still yield the same flat energy distribution if the contributions from internal bremsstrahlung and $\tau$ lepton decays are included. The price to pay however is an unacceptably large mass of $10 \mathrm{TeV}$. Notice finally that a contribution from DM annihilation cannot be ruled out provided that it contributes less than $10 \%$ of the signal. 


\section{DM substructures and the galactic lottery}

In the presence of cold dark matter - which is made in our case of non-relativistic weakly interacting massive particles or WIMPs - the smallest DM structures form first. They become non-linear at a red shift of order 50 to 100 and collapse to form dense virialized clumps which subsequently gather inside larger and larger objects until the halos of galaxies appear. At the same time, baryons fall inside the DM potential wells where they lead to the first stars and globular clusters. This hierarchical scenario is very reminiscent of matryoshka dolls designed to nest inside one another in the same way as the smallest DM substructures do inside the larger halos which they contribute to build. The size of the smallest DM subhalos is fixed by the free-streaming length over which WIMPs have been able to travel freely since the big bang, erasing any primordial density fluctuation. As long as the DM species are thermally coupled with the primordial plasma, their propagation is severely hampered by the numerous elastic collisions which they undergo. As thermal freeze-out takes place - as a result of cooling and dilution [117] - the diffusion of WIMPs stops. The particles become free to move, albeit very slowly since they are already non-relativistic. A general discussion of these effects can be found in [118]. The free-streaming length sets a lower bound on the mass of the DM substructures which depends on the nature and properties of the WIMP $[119,120]$. Typical values range from $3 \times 10^{-9} M_{\odot}$ up to $0.3 M_{\odot}$ [121]. As the cosmic web evolves, the DM structures merge and can also be partially disrupted by galactic tidal forces. The current cutoff in the spectrum of clump masses corresponds to the smallest surviving objects and is not yet well understood. According to numerical simulations [122, 123, 124], DM subhalos as light as $10^{-6} M_{\odot}$ can survive at low red shift whereas analytical considerations [125] point toward the disruption of objects smaller than $10^{3} M_{\odot}$. The possibility that the impulsive encounters with stars alongside disc shocking and tidal stripping could be a serious threat to the survival of primordial microhalos has also been hotly debated - see for instance [126, 127] for a discussion of these effects and [128] for a more optimistic conclusion. It is fair to say that no definite conclusion has yet been reached as regards the mass spectrum, the morphology and the galactic distribution of DM substructures. The presence of the latter in DM halos is nevertheless commonly accepted now with its immediate consequence that the annihilation rate of WIMPs is enhanced.

Exercise $\mathbf{n}^{0}$ 3-a - Level [1] : Let us consider a domain encompassing a volume $\mathscr{V}$ inside which DM particles are not distributed homogeneously. Their number density $n_{\chi}(\mathbf{x})$ depends thus on the location $\mathbf{x}$ within the volume $\mathscr{V}$. As mentioned above, this is typically the case of a clumpy DM galactic halo. Should WIMPs be homogeneously distributed, their numerical density would be given by the average

$$
\left\langle n_{\chi}\right\rangle=\frac{1}{\mathscr{V}} \int_{\mathscr{Y}} n_{\chi}(\mathbf{x}) d^{3} \mathbf{x} .
$$

Show that density fluctuations boost the annihilation rate of DM species by a factor

$$
\mathscr{C}=\left\langle n_{\chi}^{2}\right\rangle /\left\langle n_{\chi}\right\rangle^{2} \geq 1
$$

where

$$
\left\langle n_{\chi}^{2}\right\rangle=\frac{1}{\mathscr{V}} \int_{\mathscr{Y}} n_{\chi}^{2}(\mathbf{x}) d^{3} \mathbf{x}
$$


Because clumpiness enhances the annihilation rate of DM particles, it also increases the corresponding indirect signatures and leads a priori to better detection prospects. In most studies however, this effect is taken into account by an energy-independent multiplicative factor called boost factor by which the signal computed from a smooth DM distribution should be multiplied. The values quoted are in general put by hand and generous enough to get a detectable flux overcoming the background - see sections 2.2 and 2.3. This has been shown to be wrong in a recent statistical analysis [87]. The Green function connecting the production of the various cosmic radiations to their observation at the Earth introduces actually a dependence on the energy.
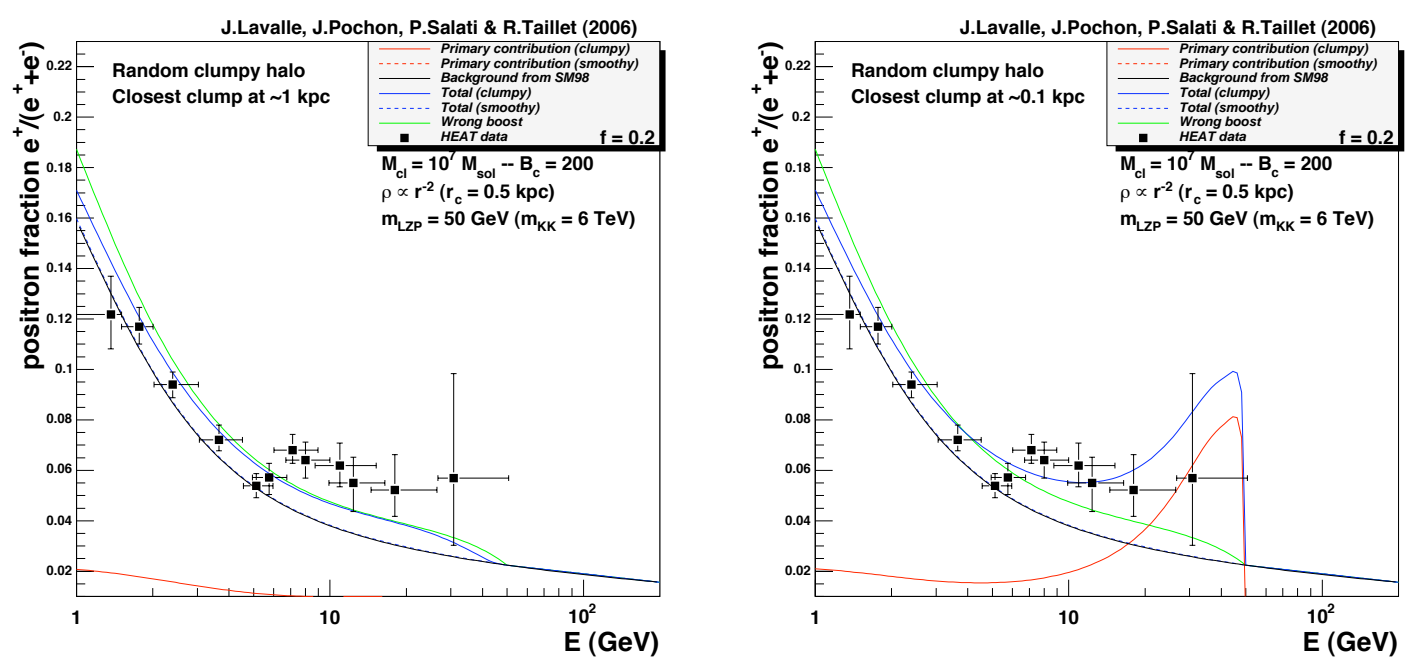

Figure 27: The positron fraction is derived for a $50 \mathrm{GeV}$ Kaluza-Klein inspired particle [129] and compared to the HEAT excess [97] which cannot be explained by the pure conventional background [89] in black. An isothermal profile has been assumed for the dark matter halo of the Milky Way, a fraction $f=0.2$ of which lies in clumps with mass $10^{7} M_{\odot}$ and intrinsic boost $B_{c}=200$. Two random realizations of that clumpy $\mathrm{DM}$ halo are featured in blue. The distance of the closest substructure has been set equal to $1 \mathrm{kpc}$ in the left panel and decreased to $0.1 \mathrm{kpc}$ in the right panel. The green curve corresponds to the traditional and wrong shift by a factor of $f \times B_{c}=40$ of the positron spectrum which a completely smooth DM halo would yield. Figure from [87].

Moreover, a unique number cannot account for an effect which turns out to be stochastic as a result of our ignorance. Should we know the exact location of each DM substructure inside the Milky Way halo, we would be able to derive the corresponding flux at the Earth. However, this is not the case even if general hypotheses about the statistical properties of the distribution of DM clumps can be made. In some cases, the expected signal from a given type of DM particle can be quite sensitive to the precise position of the nearest subhalos relative to the Earth. As an illustration, the possibility that the positron excess observed by HEAT could be due to a single nearby clump has been raised in $[130,131]$. The corresponding probability was estimated to be of order $10^{-4}$ in the case of a supersymmetric WIMP. In the same spirit, the case of $50 \mathrm{GeV}$ LZP species [129] is analyzed in figure 27. Each panel corresponds to the same Monte Carlo realization of a clumpy halo made of $10^{7} M_{\odot}$ objects. The only difference lies in the distance of the closest clump to the Earth. Decreasing that distance from $1 \mathrm{kpc}$ down to $100 \mathrm{pc}$ has dramatic consequences on the positron excess as is clear in the right panel. Finally, in the case of high-energy photons - and neutrinos - 
the enhancement of the signal depends also on the direction. Subhalos are more easily destroyed as they wander close to the galactic center $[132,133]$ and the boost factor should be depressed in that direction.

\subsection{A statistical approach of galactic variance}

Should a DM substructure lie in our immediate vicinity, it would emit a strong signal in a variety of cosmic ray channels. As shown in figure 27, we would observe for instance a significantly distorted positron spectrum in the case of a LZP species. But how probable is this?

To answer that question, a statistical analysis becomes mandatory. We would like to relate the stochastic distribution of DM clumps within the Galaxy to the indirect signals which these subhalos would generated at the Earth. In the absence of any substructure, the DM distribution $\rho_{\text {smth }}$ is smooth and its radial profile (2.34) or (2.109) is specified in tables 6 or 7 . The flux at the Earth yielded by the annihilating DM particles can be expressed as

$$
\Phi_{\text {smth }}(\odot, E)=\mathscr{F} \times\left\{\mathbb{I}_{\text {smth }}^{(2)} \equiv \int_{\mathrm{DH}} d^{3} \mathbf{x} \mathscr{G}\left(\mathbf{x}_{\odot} \leftarrow \mathbf{x}\right) \frac{\rho_{\mathrm{smth}}^{2}(\mathbf{x})}{\rho_{\odot}^{2}}\right\}
$$

where relation (2.8) has been adapted in order to put the stress on the position of the sources. The multiplicative factor $\mathscr{F}$ depends on the mass and annihilation cross section of the WIMP and is defined in relation (2.9). The cosmic ray energy distribution $f\left(E_{S}\right)$ at the source as well as the energy $E$ of detection are now embedded into the effective Green function

$$
\mathscr{G}\left(\mathbf{x}_{\odot} \leftarrow \mathbf{x}_{S}\right) \equiv \int d E_{S} f\left(E_{S}\right) G\left(\mathbf{x}_{\odot}, E \leftarrow \mathbf{x}_{S}, E_{S}\right)
$$

In the case of antiprotons for which energy losses and diffusive reacceleration are negligible above a few $\mathrm{GeV}$, the effective propagator $\mathscr{G}$ boils down to the function $G_{\overline{\mathrm{p}}}\left(\mathbf{x}_{\odot} \leftarrow \mathbf{x}_{S}, E\right)$ of equation (2.23). For DM particles annihilating into electron-positron pairs - direct production is presented in the upper left panel of figure 26 - the effective Green function is identical to the positron propagator $G_{e^{+}}\left(\mathbf{x}_{\odot}, E \leftarrow \mathbf{x}_{S}, m_{\chi}\right)$ defined in relation (2.59). A continuous energy spectrum $f\left(E_{S}\right)$ at the source arises for the other WIMP annihilation channels and leads to

$$
\mathscr{G}\left(\mathbf{x}_{\odot} \leftarrow \mathbf{x}_{S}\right)=\int_{E}^{m_{\chi}} d E_{S} f\left(E_{S}\right) G_{e^{+}}\left(\mathbf{x}_{\odot}, E \leftarrow \mathbf{x}_{S}, E_{S}\right)
$$

Whatever the cosmic ray particle, we will keep in mind that the effective propagator $\mathscr{G}$ varies with energy $E$ even though we will be more interested here in its dependence on position. In the presence of clumps, the DM distribution is given by the superposition

$$
\rho=\rho_{\text {smth }}^{\prime}+\delta \rho
$$

where the smooth part corresponds now to $\rho_{\text {smth }}^{\prime}$ whereas $\delta \rho$ stands for the substructures. DM subhalos are assumed to be quite concentrated. The distribution $\delta \rho$ vanishes everywhere except in very localized regions where it overcomes completely the component $\rho_{\text {smth }}^{\prime}$. 
Exercise $\mathbf{n}^{0}$ 3-b - Level [1] : The antiproton and positron cosmic ray horizons are much more extended than the DM substructures. The latter can be considered as point-like objects. Compute then the flux at the Earth yielded by the distribution 3.7 in order to establish that

$$
\Phi(\odot, E)=\Phi_{\text {smth }}^{\prime}(\odot, E)+\left\{\Phi_{\text {rand }}(\odot, E) \equiv \sum_{i} \varphi_{i}\right\},
$$

where the sum runs over the population of subhalos. Show that the contribution from the ith clump located at $\mathbf{x}_{i}$ is

$$
\varphi_{i}=\mathscr{F} \times \mathscr{G}\left(\mathbf{x}_{\odot} \leftarrow \mathbf{x}_{i}\right) \times \xi_{i},
$$

where the volume $\xi_{i}$ is defined by

$$
\xi_{i} \equiv \int_{\text {ith clump }} d^{3} \mathbf{x}\left\{\frac{\delta \rho(\mathbf{x})}{\rho_{\odot}}\right\}^{2} .
$$

The ith minihalo produces as many cosmic ray species as if its so-called annihilation or solar equivalent volume were filled with an homogeneous distribution of the same DM species at a density equal to the local value $\rho_{\odot}$. The intrinsic boost factor $B_{i}-$ with respect to the solar DM environment - can be defined by

$$
\xi_{i}=\frac{B_{i} M_{i}}{\rho_{\odot}}
$$

where $M_{i}$ stands for the mass of the substructure. The intrinsic boost $B_{i}$ characterizes the enhancement of the annihilation rate inside the ith subhalo with respect to the situation where that object would be uniformely spread in the solar neighborhood with the inner density $\rho_{\text {. }}$. The boost factor of the cosmic ray signal at the Earth is defined as

$$
B(E) \equiv \frac{\Phi(\odot, E)}{\Phi_{\text {smth }}(\odot, E)}=\frac{\Phi_{\text {smth }}^{\prime}(\odot, E)}{\Phi_{\text {smth }}(\odot, E)}+\frac{\Phi_{\text {rand }}(\odot, E)}{\Phi_{\text {smth }}(\odot, E)}
$$

It measures the increase of the flux to which clumpiness leads with respect to the smooth DM distribution $\rho_{\text {smth }}$. This ratio depends on the energy $E$ through the cosmic ray propagator $\mathscr{G}$. It is also very sensitive to the actual position of the subhalos whose contribution $\Phi_{\text {rand }}$ can only be accessed statistically as a result of our ignorance of their exact location within the Milky Way. Not only does $B$ depend on the energy of detection $E$, but it definitely must be analyzed within a stochastic framework.

To do so, an infinite set of halo realizations must be taken into account, each of which produces a different flux $\Phi_{\text {rand }}$. The boost factor is not unique and must be treated as a random variable. The procedure to determine the statistical law according to which $B$ is distributed has been thoroughly discussed in [87]. We will just point out the salient features of this analysis and summarize the hypotheses on which it is based.

(i) The actual distribution of DM substructures is one particular realization to be taken from the statistical ensemble made up by all the possible random distributions. The flux contributed by the 


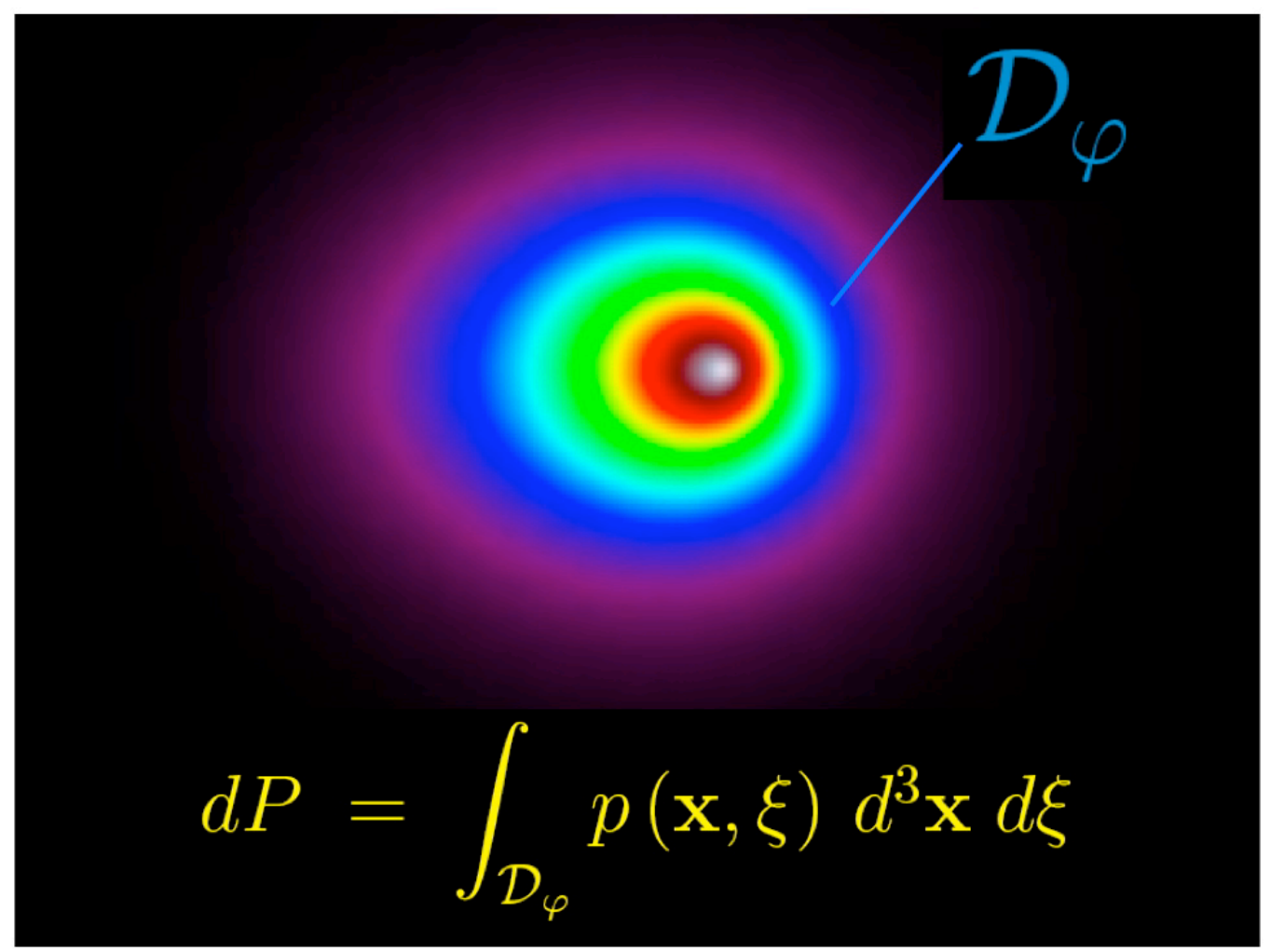

Figure 28: The parameter space is schematically represented in this diagram. A point in that space is characterized by the position $\mathbf{x}$ of the DM substructure within the galactic halo $\mathscr{D}_{H}$ and by its annihilation volume $\xi$. Each region corresponds to a specific flux which the clump generates at the Earth. The intensity of the signal is coded with different colors. The probability to get a flux $\varphi$ is given by the integral of the distribution function $p(\mathbf{x}, \xi)$ over the domain $\mathscr{D}_{\varphi}$ of parameter space which yields the required value.

clumps must be averaged over that infinite set to yield $\left\langle\Phi_{\text {rand }}\right\rangle$ and the associated variance is defined by the canonical relation

$$
\sigma_{\text {rand }}^{2}=\left\langle\Phi_{\text {rand }}^{2}\right\rangle-\left\langle\Phi_{\text {rand }}\right\rangle^{2} .
$$

This translates into the average boost factor

$$
B_{\mathrm{eff}} \equiv\left\langle B=\left(\Phi / \Phi_{\mathrm{smth}}\right)\right\rangle
$$

and boost variance

$$
\sigma_{B}=\sigma_{\text {rand }} / \Phi_{\text {smth }}
$$

(ii) Clumps are distributed independently of each other. This is a strong assumption that generally holds because the substructure two-body correlation length is smaller than the cosmic ray horizon size set, for instance, by the propagation range $\lambda_{\mathrm{D}}$ in the case of positrons. We just need then to determine how a single clump is distributed inside the Milky Way halo in order to derive the statistical properties of an entire population of $N_{H}$ such substructures. If $\varphi$ denotes the contribution of a single object, the average contribution of the DM minihalos to the flux is equal to

$$
\left\langle\Phi_{\text {rand }}\right\rangle=N_{H} \times\langle\varphi\rangle,
$$


while its variance can be expressed as

$$
\sigma_{\text {rand }}^{2}=N_{H} \times \sigma^{2} \equiv N_{H} \times\left\{\left\langle\varphi^{2}\right\rangle-\langle\varphi\rangle^{2}\right\}
$$

(iii) The set of the random distributions of one single clump inside the Milky Way halo - a domain $\mathscr{D}_{H}$ beyond which the signal is no longer detectable at the Earth and large enough to encompass the DH in the case of charged cosmic rays - makes up the statistical ensemble which we eventually need to consider. An event from that ensemble consists in the presence of a clump characterized by the annihilation volume $\xi$ - up to $d \xi$ - and located at position $\mathbf{x}$ - within the elementary volume $d^{3} \mathbf{x}$. The distribution function $p(\mathbf{x}, \xi)$ with which the events are weighted is related to the probability $\mathscr{P}(\varphi)$ to get the flux $\varphi$ insofar as

$$
\mathscr{P}(\varphi) d \varphi=d P=\int_{\mathscr{D}_{\varphi}} p(\mathbf{x}, \xi) d^{3} \mathbf{x} d \xi
$$

The region of parameter space, namely the position $\mathbf{x}$ inside the galactic halo $\mathscr{D}_{H}$ and the annihilation volume $\xi$, inside which a clump must lie in order to contribute a flux $\varphi$ at the Earth is denoted by $\mathscr{D}_{\varphi}$. We readily conclude that any function $\mathscr{H}$ of the flux $\varphi$ is given on average by the convolution

$$
\langle\mathscr{H}\rangle=\int \mathscr{H}(\varphi) \mathscr{P}(\varphi) d \varphi \equiv \int_{\mathscr{D}_{H}} \mathscr{H}\{\varphi(\mathbf{x}, \xi)\} \times p(\mathbf{x}, \xi) \times d^{3} \mathbf{x} d \xi
$$

This is true in particular for the flux $\varphi$ itself or its square, hence the possibility to compute the average quantities $\langle\varphi\rangle$ and $\left\langle\varphi^{2}\right\rangle$ which come into play in relations (3.16) and (3.17).

(iv) Once the single clump distribution function $p(\mathbf{x}, \xi)$ has been determined, we are naturally lead to the effective boost factor

$$
B_{\text {eff }}=\left\{\frac{\Phi_{\text {smth }}^{\prime}}{\Phi_{\text {smth }}} \simeq 1\right\}+\frac{\left\langle\Phi_{\text {rand }}\right\rangle}{\Phi_{\text {smth }}}=1+N_{H} \times \frac{\langle\xi \mathscr{G}\rangle}{\mathbb{I}_{\text {smth }}^{(2)}},
$$

and to the boost variance

$$
\frac{\sigma_{B}}{B_{\text {eff }}}=\frac{\sigma_{\text {rand }} / \Phi_{\text {smth }}}{1+\left\langle\Phi_{\text {rand }}\right\rangle / \Phi_{\text {smth }}} \simeq \frac{\sigma_{\text {rand }}}{\left\langle\Phi_{\text {rand }}\right\rangle}
$$

The relative variance of the random part of the signal can be derived from the generic expresion (3.19) which, together with the definitions (3.16) and (3.17), leads to

$$
\left\{\frac{\sigma_{\text {rand }}}{\left\langle\Phi_{\text {rand }}\right\rangle}\right\}^{2}=\frac{1}{N_{H}} \times\left\{\frac{\left\langle\xi^{2} \mathscr{G}^{2}\right\rangle}{\langle\xi \mathscr{G}\rangle^{2}}-1\right\} .
$$

This statistical method has been applied in [87] to configurations where the clumps are assumed to be identical objects. To simplify the discussion and without loss of generality, we will consider hereafter subhalos with the same mass $M_{c}$, the same annihilation volume $\xi_{c}$ and thus the same intrinsic boost

$$
B_{c}=\frac{\xi_{c} \rho_{\odot}}{M_{c}} .
$$

In the example featured in figure 27, each substructure has a mass of $10^{7} M_{\odot}$ and its intrinsic boost $B_{c}$ is equal to 200 . 
Exercise $\mathbf{n}^{0}$ 3-c - Level [2] : We will also consider that a fraction $f$ of the completely smooth DM distribution $\rho_{\text {smth }}$ has actually collapsed in substructures which follow the same pattern. What remains of the smooth component is given by

$$
\rho_{\text {smth }}^{\prime} \equiv(1-f) \times \rho_{\text {smth }},
$$

whereas the density of the subhalos is equal on average to

$$
\langle\delta \rho\rangle \equiv f \times \rho_{\text {smth }} .
$$

The Milky Way halo $\mathscr{D}_{H}$ contains a large number $N_{H}$ of identical DM clumps which are characterized by their mass $M_{c}$ and intrinsic boost $B_{c}$. The distribution function that describes the position and annihilation volume of a single minihalo may be expressed as

$$
p(\mathbf{x}, \xi) \equiv p(\mathbf{x}) \times \delta\left(\xi-\xi_{c}\right) .
$$

Show that the average number density of clumps within the galactic halo can be expressed as

$$
n(\mathbf{x})=N_{H} p(\mathbf{x})=\frac{f \rho_{\mathrm{smth}}(\mathbf{x})}{M_{c}} .
$$

The ratio $\Phi_{\text {smth }}^{\prime} / \Phi_{\text {smth }}$ is smaller than 1 and is in general negligible with respect to $\left\langle\Phi_{\text {rand }}\right\rangle / \Phi_{\text {smth }}$. Express that ratio in terms of the fraction $f$ and establish that the effective boost is equal to

$$
B_{\mathrm{eff}}=(1-f)^{2}+f B_{c} \times \frac{\mathbb{I}_{\mathrm{smth}}^{(1)}}{\mathbb{I}_{\mathrm{smth}}^{(2)}},
$$

where the integral $\mathbb{I}_{\text {smth }}^{(\mathrm{n})}$ is generically defined as

$$
\mathbb{I}_{\text {smth }}^{(\mathrm{n})} \equiv \int_{\mathrm{DH}} d^{3} \mathbf{x} \mathscr{G}\left(\mathbf{x}_{\odot} \leftarrow \mathbf{x}\right)\left\{\frac{\rho_{\text {smth }}(\mathbf{x})}{\rho_{\odot}}\right\}^{\mathrm{n}} .
$$

Show that the relative variance of the boost is given now exactly by

$$
\frac{\sigma_{B}}{B_{\text {eff }}}=\frac{\sigma_{\text {rand }} / \Phi_{\text {smth }}}{(1-f)^{2}+\left\langle\Phi_{\text {rand }}\right\rangle / \Phi_{\text {smth }}} \simeq \frac{\sigma_{\text {rand }}}{\left\langle\Phi_{\text {rand }}\right\rangle} .
$$

The product of the fraction $f$ by the intrinsic boost $B_{c}$ is in general much larger than 1 whereas the ratio $\mathbb{I}_{\text {smth }}^{(1)} / \mathbb{I}_{\text {smth }}^{(2)}$ is of order unity. Remember that subhalos have the same annihilation volume $\xi_{c}$ and demonstrate that

$$
\left\{\frac{\sigma_{B}}{B_{\text {eff }}}\right\}^{2} \simeq\left\{\frac{\sigma_{\text {rand }}}{\left\langle\Phi_{\text {rand }}\right\rangle}\right\}^{2}=\frac{1}{N_{H}} \times\left\{\frac{\left\langle\mathscr{G}^{2}\right\rangle}{\langle\mathscr{G}\rangle^{2}}-1\right\} .
$$

Apply this relation to our case in order to derive the boost variance

$$
\left\{\frac{\sigma_{B}}{B_{\text {eff }}}\right\}^{2} \simeq \frac{M_{c}}{f \rho_{\odot}} \times \mathbb{J}_{\text {smth }}^{(1)} \times\left\{\mathbb{I}_{\text {smth }}^{(1)}\right\}^{-2}-\frac{1}{N_{H}}
$$

where the integral $\underset{\mathrm{J}}{\mathrm{smth}}(\mathrm{n})$ is given by

$$
\mathbb{J}_{\mathrm{smth}}^{(\mathrm{n})} \equiv \int_{\mathrm{DH}} d^{3} \mathbf{x} \mathscr{G}^{2}\left(\mathbf{x}_{\odot} \leftarrow \mathbf{x}\right)\left\{\frac{\rho_{\mathrm{smth}}(\mathbf{x})}{\rho_{\odot}}\right\}^{\mathrm{n}} .
$$


The discussion will be focused on DM species annihilating directly into electron-positron pairs. The latter particles are monochromatic at the source as they are produced with the energy $E_{S} \equiv m_{\chi}$. The positron line is indeed a prototypical example. It leads to a cosmic ray horizon whose extension around the Earth is gauged by the diffusion length $\lambda_{\mathrm{D}}$ and depends on whether the energy $E$ of detection is close to $E_{S}$ or not. Moreover, the effective Green function $\mathscr{G}$ which comes into play in the integrals $\mathbb{I}_{\text {smth }}^{(\mathrm{n})}$ and $\mathbb{J}_{\text {smth }}^{(\mathrm{n})}$ can be replaced by the positron propagator $G_{e^{+}}$whose construction is completed in section 2.3. After a close inspection of relations (3.28) and (3.32), we may even substitute $\mathscr{G}$ with the reduced propagator $\tilde{G}$ of equation (2.59). The relevant energy variable of the integrals $\mathbb{I}_{\text {smth }}^{(\mathrm{n})}$ and $\mathbb{J}_{\text {smth }}^{(\mathrm{n})}$ is thus the positron diffusion length $\lambda_{\mathrm{D}}$. The same remark applies to the effective boost $B_{\text {eff }}$ and to the variance $\sigma_{B}$. Both depend on the energies $E$ and $E_{S}$ through the length $\lambda_{\mathrm{D}}$.

The case of a $100 \mathrm{GeV}$ positron line is shown in figure 29. A fraction $f=0.2$ of the DM halo is in the form of substructures whose intrinsic boost $B_{c}$ is equal to 100 . In the numerical simulations of [122], such a value for the fraction $f$ would correspond to a minimum mass scale of $10^{4} M_{\odot}$. As for the boost $B_{c}$, the values quoted in the literature vary from a few [125] up to over two orders of magnitude [122]. The black central curve features the effective boost $B_{\text {eff }}$ of our clumpy halo with respect to the NFW profile of table 7 whose scale factor $r_{\mathrm{s}}$ has been raised to $25 \mathrm{kpc}$. The increase of $B_{\text {eff }}$ with positron energy is clear. Near the positron line, in the region where $E$ is close to the input energy $E_{S}$, the reduced Green function $\tilde{G}$ probes only a small region of the Milky Way halo around the solar system. The ratio $\mathbb{I}_{\text {smth }}^{(1)} / \mathbb{I}_{\text {smth }}^{(2)}$ boils down to unity and expression (3.28) simplifies into $\sim f B_{c}$. That is why the effective boost reaches a value of $\sim 20$ as $E$ approaches the injection energy of $100 \mathrm{GeV}$. If now $E$ is varied from its upper limit $E_{S}$ downward, larger portions of the halo are taken into account in the integrals $\mathbb{I}_{\text {smth }}^{(1)}$ and $\mathbb{I}_{\text {smth }}^{(2)}$ whose ratio is found numerically to decrease. Regions located close to the galactic center come into play, with a much larger density $\rho_{\mathrm{smth}}$ than in the solar neighborhood. At fixed intrinsic boost $B_{c}$, neutralino annihilation inside DM substructures is relatively less enhanced in these dense regions than in our vicinity, hence a lower value of the effective boost at low energy than near the positron line. Another characteristic feature of figure 29 is the tulip shape of the $1-\sigma$ range of the effective boost fluctuations. That range extends from $B_{\text {min }}=B_{\text {eff }}-\sigma_{B}$ up to $B_{\text {max }}=B_{\text {eff }}+\sigma_{B}$. Three values of the clump mass $M_{c}$ have been considered. At fixed clump mass, the tulip opens up as $E$ approaches the injection energy $E_{S}$. The fluctuations in the signal increase significantly just below the positron line. Notice also the broadening of the boost uncertainty band as the clump mass is varied from $10^{4}$ up to $10^{6} \mathrm{M}_{\odot}$.

In order to understand the enlargement of the tulip of figure 29 as the subhalo mass $M$ is varied, we need to develop the hard-sphere approximation discussed in [87]. As long as $\lambda_{\mathrm{D}}$ is small compared to the half-thickness $L$ of the cosmic ray diffusive halo, the reduced propagator $\tilde{G}$ is given by the particularly simple Gaussian function (2.46)

$$
\tilde{G}\left(\mathbf{x}_{\odot} \leftarrow \mathbf{x} ; \lambda_{\mathrm{D}}\right)=\frac{1}{\pi^{3 / 2} \lambda_{\mathrm{D}}^{3}} \exp \left\{-\frac{r_{\oplus}^{2}}{\lambda_{\mathrm{D}}^{2}}\right\}
$$

This form applies strictly for values of $E$ close to the injection energy $E_{S}$. It nevertheless provides the qualitatively correct behaviour of $\tilde{G}$ even for detection energies as small as a few GeV. Let us 


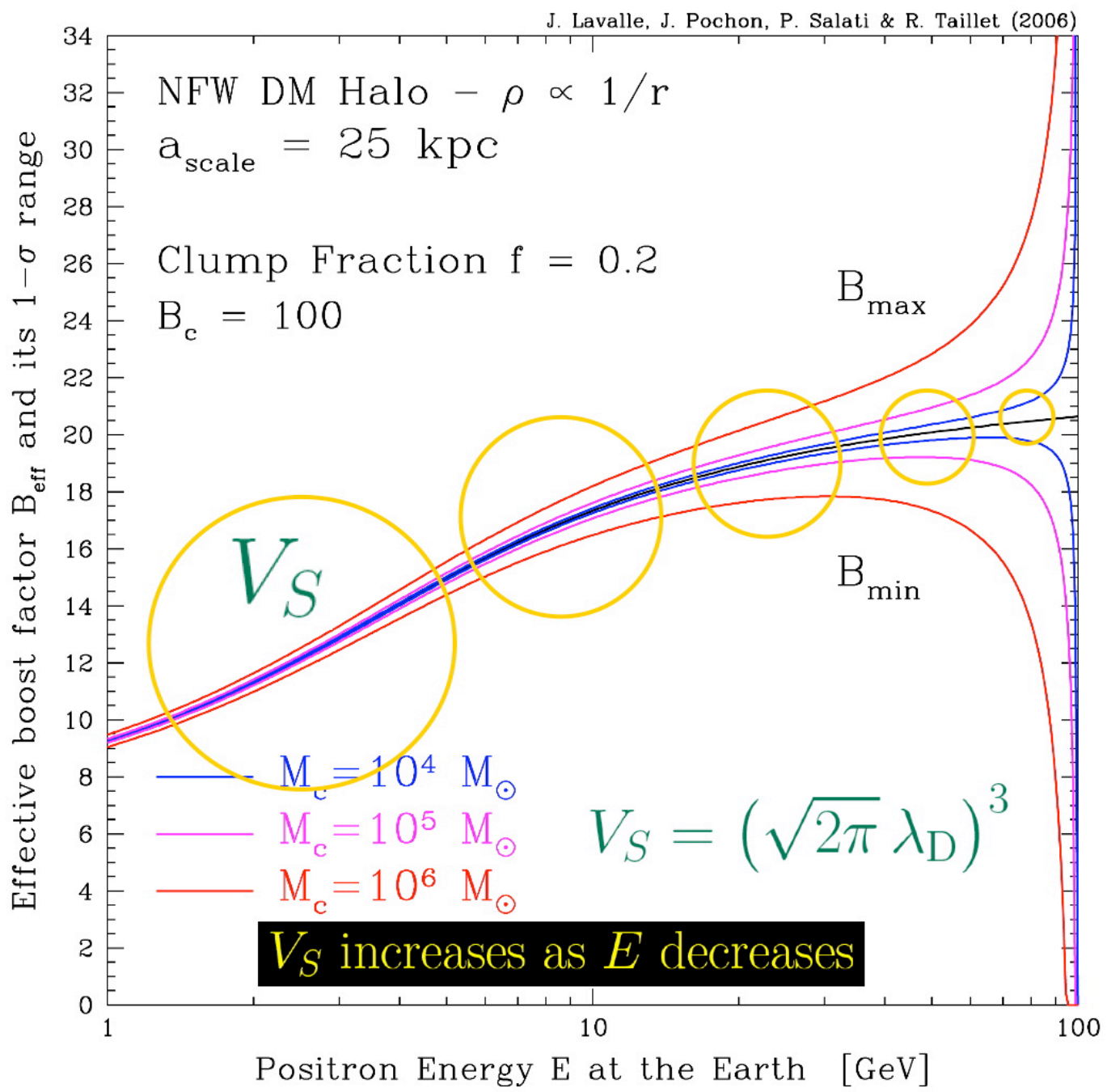

Figure 29: Positrons are injected with the energy $E_{S}=100 \mathrm{GeV}$ and detected at energy $E$. The effective boost factor $B_{\text {eff }}$ (black solid line) accounts for the average enhancement of the positron signal resulting from DM clumpiness. Although it has been so far considered to behave as a constant, the boost actually depends on the energy. Furthermore, as $E$ approaches $E_{S}$, the boost variance $\sigma_{B}$ increases significantly. The region from which the positrons detected at the Earth originate shrinks and the number $N_{S}$ of clumps which it contains decreases. Figure from [87].

simplify even further our analysis by substituting the step function

$$
\tilde{G}\left(\mathbf{x}_{\odot} \leftarrow \mathbf{x} ; \lambda_{\mathrm{D}}\right)=\frac{\theta\left(r_{S}-r_{\oplus}\right)}{V_{S}}
$$

for the Gaussian form (3.34). The distance between the clump and the Earth is denoted by $r_{\oplus} \equiv$ $\left|\mathbf{x}-\mathbf{x}_{\odot}\right|$. According to this hard-sphere approximation, the Green function $\tilde{G}$ is characterized by a constant value of $1 / V_{S}$ inside the sphere $\mathscr{D}_{S}$ of radius $r_{S}$ and volume $V_{S}$ and vanishes elsewhere. Both expressions (3.34) and (3.35) are normalized to unity. The integral over the full 3D space of the square of those Green functions should also be the same. 
Exercise $\mathbf{n}^{0}$ 3-d - Level [1] : Show that this condition implies that

$$
\frac{1}{V_{S}}=\int \tilde{G}^{2} d^{3} \mathbf{x}
$$

and leads to the horizon volume

$$
V_{S}=\left(\sqrt{2 \pi} \lambda_{\mathrm{D}}\right)^{3}
$$

In the limit where $\lambda_{\mathrm{D}}$ is small with respect to $L$, the hard-sphere model provides a quantitatively correct framework and the integrals $\mathbb{J}_{\text {smth }}^{(1)}$ and $\mathbb{I}_{\text {smth }}^{(1)}$ simplify. The positron horizon is limited and the DM smooth density $\rho_{\text {smth }}(\mathbf{x})$ can be taken equal to $\rho_{\odot}$ everywhere inside $\mathscr{D}_{S}$. The integral $\mathbb{I}_{\text {smth }}^{(1)}$ is equal to unity whereas $\mathbb{J}_{\text {smth }}^{(1)}$ is given by the ratio $1 / V_{S}$ as shown in the previous exercise. We may thus calculate directly the boost variance from relation (3.32) and get

$$
\left\{\frac{\sigma_{B}}{B_{\text {eff }}}\right\}^{2} \simeq \frac{M_{c}}{f \rho_{\odot} V_{S}}-\frac{1}{N_{H}} .
$$

The DM density in the form of substructures is equal on average to $f \rho_{\odot}$ in the solar neighborhood. The subhalos lying within the positron sphere contribute on average a total mass $M_{S} \equiv f \rho_{\odot} V_{S}$ and their number is given by

$$
N_{S}=\frac{f \rho_{\odot} V_{S}}{M_{c}} .
$$

This number refers to the objects contributing effectively to the positron signal at the Earth. It is very small with respect to the total number $N_{H}$ of DM clumps contained in the Milky Way halo $\mathscr{D}_{H}$. The boost variance simplifies into

$$
\frac{\sigma_{B}}{B_{\text {eff }}} \simeq \frac{\sigma_{\text {rand }}}{\left\langle\Phi_{\text {rand }}\right\rangle}=\sqrt{\frac{M_{c}}{f \rho_{\odot} V_{S}}}=\frac{1}{\sqrt{N_{S}}},
$$

and may be interpreted as the Poisson noise associated to the random presence of subhalos inside the positron sphere. The broadening of the tulip in figure 29 is associated to the decrease of the number $N_{S}$ of minihalos which contribute to the signal. As $E$ approaches $E_{S}$, the positron sphere shrinks. It contains fewer sources and the Poisson noise increases. The enlargement of the tulip as the clump mass is varied can also be explained in the same way. It translates the proportionality between $\sigma_{B}$ and $\sqrt{M_{c}}$. The heavier the subhalos, the fewer within the positron horizon and the larger the variance.

In spite of its crudeness, the hard-sphere approximation turns out to be an excellent tool to understand the statistical properties of both the clump distribution and its flux. The probability $p$ that a subhalo lies inside the positron sphere $\mathscr{D}_{S}$ - from which it may yield a signal at the Earth - is just the ratio $N_{S} / N_{H}$ of the average number $N_{S}$ of objects confined in that region over the total number of substructures $N_{H}$ contained in the entire domain $\mathscr{D}_{H}$. For an injected energy $E_{S}=100 \mathrm{GeV}$ and a positron energy at the Earth $E=50 \mathrm{GeV}$, we find a probability $p \sim 2 \times 10^{-3}$ when the domain $\mathscr{D}_{H}$ is chosen to be the above-mentioned NFW halo with a radial extension limited at $20 \mathrm{kpc}$ from 
the center of the Milky Way. Because $p$ is vanishingly small and the total number of clumps $N_{H}$ exceedingly large, the limit of Poisson statistics is reached. The probability to find $n$ subhalos inside the positron sphere $\mathscr{D}_{S}$ is given by

$$
P(n)=\frac{N_{S}{ }^{n}}{n !} \exp \left(-N_{S}\right)
$$

where $\langle n\rangle \equiv N_{S}$ is the average number of clumps contributing to the signal. That expression has already been established in section 1.3 - see relation (1.90). The Poisson distribution (3.41) is associated to the variance

$$
\sigma_{n}^{2}=\left\langle n^{2}\right\rangle-\langle n\rangle^{2}=N_{S}
$$

In the hard-sphere approximation, the random part $\Phi_{\text {rand }}$ of the positron flux at the Earth - the contribution which the entire constellation of substructures generates - is proportional to the number $n$ of clumps lying inside the sphere $\mathscr{D}_{S}$. That is why the relative variance $\sigma_{\text {rand }} /\left\langle\Phi_{\text {rand }}\right\rangle$ is exactly equal in this regime to the variance $\sigma_{n} /\langle n\rangle$ associated to the Poisson law (3.41). Departures from the latter occur of course in the case of a realistic positron propagator and require a careful investigation. But the statistical behaviour of the number of subhalos which effectively contribute to the signal can still efficiently drive our intuition.

The large $N_{S}$ regime

When the number $N_{S}$ of clumps involved in the flux at the Earth is large, the Poisson law (3.41) becomes the Gaussian distribution

$$
P(\delta)=\frac{1}{\sqrt{2 \pi N_{S}}} \exp \left(-\delta^{2} / 2 N_{S}\right)
$$

where $\delta \equiv n-N_{S}$ denotes the departure of the number $n$ of substructures inside the positron horizon $\mathscr{D}_{S}$ from its average value $N_{S}$. The associated variance is $\sigma_{n}=\sqrt{N_{S}}$. We therefore anticipate that the flux $\Phi_{\text {rand }}$ will also be randomly distributed according to a Gaussian law with mean value $\left\langle\Phi_{\text {rand }}\right\rangle$ and variance $\sigma_{\text {rand }}$. In order to determine the distribution of probability $\mathscr{P}\left(\Phi_{\text {rand }}\right)$ that drives the flux generated by the entire constellation of the clumps lying inside the reservoir $\mathscr{R}$, we ought to compute the product of convolution of the $N_{H}$ distributions of probability $\mathscr{P}(\varphi)$ associated each to the flux $\varphi$ of a single substructure. Such a task may seem desperate. However, in the large $N_{S}$ regime, the central limit theorem may be fruitfully applied to solve that puzzle. It states that the above-mentioned inextricate product of convolution boils down indeed into a mere Gaussian distribution with mean value $\left\langle\Phi_{\text {rand }}\right\rangle \equiv N_{H} \times\langle\varphi\rangle$ and variance $\sigma_{\text {rand }}^{2} \equiv N_{H} \times\left\{\left\langle\varphi^{2}\right\rangle-\langle\varphi\rangle^{2}\right\}$. We recognize expressions (3.16) and (3.17) which can be computed as soon as the distribution function $p(\mathbf{x}, \xi)$ is known. The probability to measure a flux $\Phi_{\text {rand }}$ at the Earth may thus be expressed as

$$
\mathscr{P}\left\{\Phi_{\text {rand }} \equiv \sum_{i} \varphi_{i}\right\}=\frac{1}{\sqrt{2 \pi \sigma_{\text {rand }}^{2}}} \exp \left\{-\frac{\left(\Phi_{\text {rand }}-\left\langle\Phi_{\text {rand }}\right\rangle\right)^{2}}{2 \sigma_{\text {rand }}^{2}}\right\} .
$$


Exercise $\mathbf{n}^{0}$ 3-e - Level [1] : Derive the probability for the total positron flux $\Phi$ at the Earth to be enhanced by a factor $B$ with respect to the completely smooth $D M$ distribution $\rho_{\text {smth }}$ and establish that

$$
\mathscr{P}\left\{B \equiv \Phi / \Phi_{\text {smth }}\right\}=\frac{1}{\sqrt{2 \pi \sigma_{B}^{2}}} \exp \left\{-\frac{\left(B-B_{\text {eff }}\right)^{2}}{2 \sigma_{B}^{2}}\right\},
$$

where the boost variance $\sigma_{B}$ can be obtained from relation (3.32). Show that the reduced boost $\eta \equiv B / B_{\text {eff }}$ follows also the same type of Gaussian law

$$
\mathscr{P}\left\{\eta \equiv B / B_{\text {eff }}\right\}=\frac{1}{\sqrt{2 \pi \sigma_{\eta}^{2}}} \exp \left\{-\frac{(\eta-1)^{2}}{2 \sigma_{\eta}^{2}}\right\},
$$

with an average value of $\langle\eta\rangle=1$ and a variance $\sigma_{\eta}=\sigma_{B} / B_{\text {eff }}$ not too different from $\sigma_{\text {rand }} /\left\langle\Phi_{\text {rand }}\right\rangle$ as featured in formula (3.21).

In order to check these theoretical considerations, a Monte Carlo simulation of a clumpy Milky Way halo has been run in [87]. A thousand different realizations have been generated at random assuming a NFW DM galactic halo with a fraction $f=0.2$ of its mass in the form of $10^{5} M_{\odot}$ clumps with intrinsic boost $B_{c}=100$. In figure 30, the number of realizations is plotted as a function of the reduced boost $\eta$ for a positron energy at the Earth of $80 \mathrm{GeV}$. This distribution is the Monte Carlo counterpart of the Gaussian law (3.46) with a mean value of $\eta$ actually very close to 1 . The rms value of the histogram is equal - within half a percent - to the variance $\sigma_{\eta}=\sigma_{B} / B_{\text {eff }}=0.1097$ which is derived from relation (3.32). With an average number $N_{S}$ of 78 subhalos inside the positron horizon at $80 \mathrm{GeV}$, the hard-sphere approximation (3.40) yields a value of $1 / \sqrt{N_{S}}=0.1132$ which is also in excellent agreement with the other results. For completeness, the histogram has been independently fitted by the Gaussian distribution

$$
\mathscr{K}(\eta, \mu, \sigma)=\frac{K}{\sqrt{2 \pi \sigma^{2}}} \exp \left\{-\frac{(\eta-\mu)^{2}}{2 \sigma^{2}}\right\} .
$$

The amplitude $K$, mean value $\mu$ and variance $\sigma$ are displayed in figure 30 and the corresponding fitted Gaussian is featured by the red curve. The width of each bin is $\Delta \eta=0.04$ and since $10^{3}$ Monte Carlo realizations have been generated, a value of $K=0.04 \times 10^{3}=40$ is expected for the amplitude. This is actually the case since the fit yields $K=39.76 \pm 1.26$. The mean value $\mu$ of the Gaussian is basically equal to 1 whereas its variance $\sigma=0.1096 \pm 0.0026$ is very close to the Monte Carlo rms value and to $\sigma_{\eta}$. Because the clumps that are involved in the positron signal at the Earth are numerous - with an average number $N_{S}=78$ in this example - the central limit theorem applies and the Gaussian distribution (3.46) is an excellent description of the statistical fluctuations of the positron flux. The question arises now to understand how the distribution of probability $\mathscr{P}(\eta)$ is modified in the limit where $N_{S}$ becomes smaller than 1. 


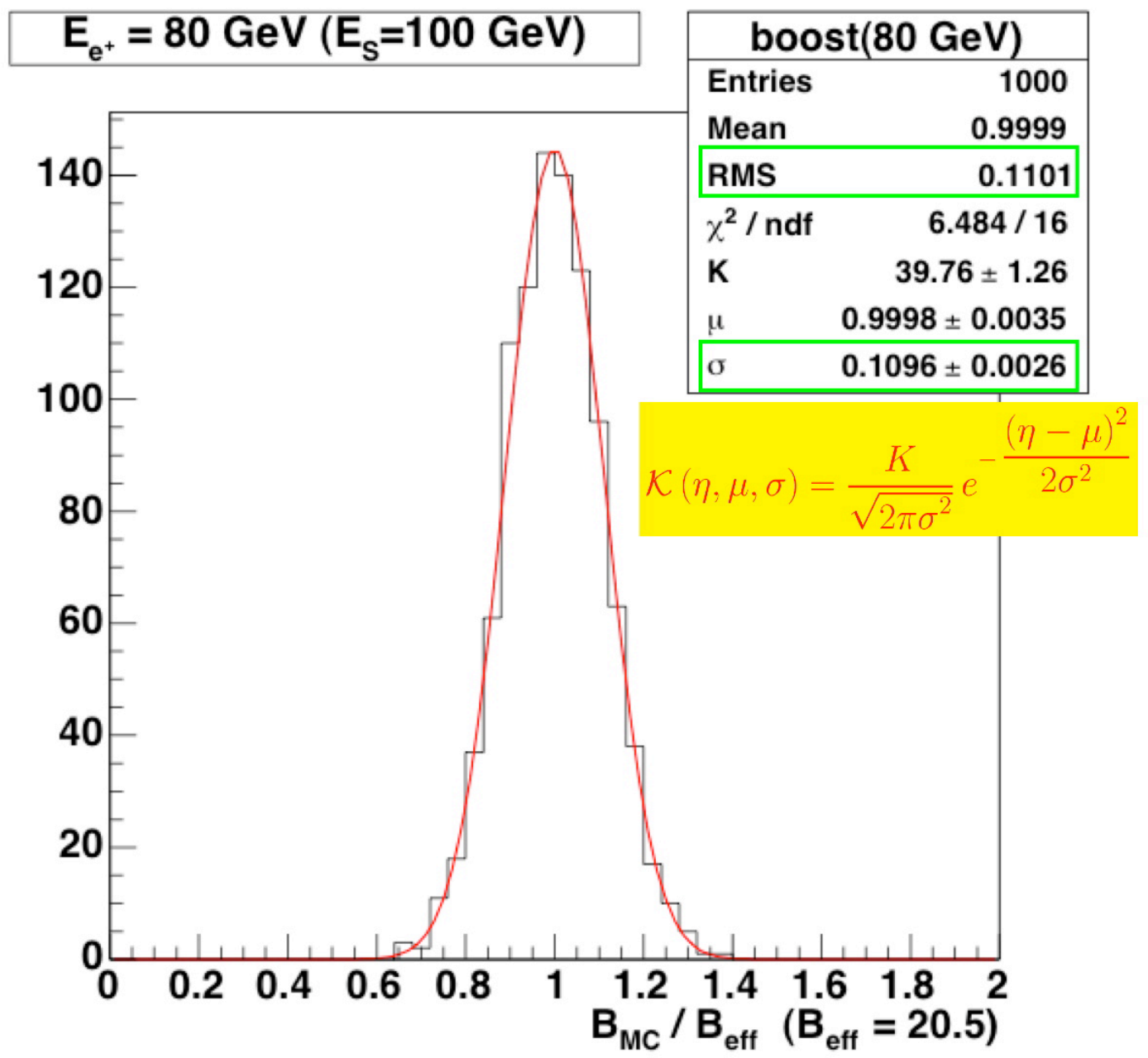

Figure 30: In this Monte Carlo simulation, substructures contribute a fraction $f=0.2$ to the mass of the Milky Way DM halo. The initial distribution $\rho_{\text {smth }}$ follows a NFW profile with a scale radius of $25 \mathrm{kpc}$. Each clump has a mass of $10^{5} M_{\odot}$ and an intrinsic boost $B_{c}=100$. A thousand different realizations of the DM halo have been generated here, each of them involving 271,488 objects. The positron injection energy is $E_{S}=100 \mathrm{GeV}$. The number of realizations has been plotted as a function of the boost ratio $\eta=B / B$ eff . The rms value of 0.1101 yielded by the Monte Carlo for $\eta$ is in excellent agreement with the anaytic value $\sigma_{\eta}=\sigma_{B} / B_{\text {eff }}=0.1097$. Figure from [87].

The small $N_{S}$ regime

When the diffusion range $\lambda_{\mathrm{D}}$ is small - for energies close to the emission energy - the positron sphere shrinks and contains on average very few subhalos. If the previous Monte Carlo analysis is performed with a clump mass of $10^{7} M_{\odot}$, the number $N_{S}$ of objects yielding a signal at $90 \mathrm{GeV}$ is equal on average to 0.242 . When a substructure is very close to the Earth, its flux $\varphi$ reaches a maximal value $\varphi_{\max }$ which depends on the properties of the clump through its mass $M_{c}$ and intrinsic boost $B_{c}$ as well as on the specific features assumed for the WIMP through the factor $\mathscr{F}$

$$
\varphi_{\max }=\mathscr{F} \times \mathscr{G}\left(\mathbf{x}_{\odot} \leftarrow \mathbf{x}_{\odot}\right) \times \frac{B_{c} M_{c}}{\rho_{\odot}}
$$


Without any loss of generality, the discussion can be significantly simplified by considering the ratio

$$
\psi(\mathbf{x})=\left\{\varphi(\mathbf{x}) / \varphi_{\max }\right\}=\left\{\mathscr{G}\left(\mathbf{x}_{\odot} \leftarrow \mathbf{x}\right) / \mathscr{G}\left(\mathbf{x}_{\odot} \leftarrow \mathbf{x}_{\odot}\right)\right\} .
$$

Because we are interested in the production of monochromatic positrons, this ratio may be further simplified into

$$
\psi(\mathbf{x})=\left\{\tilde{G}\left(\mathbf{x}_{\odot} \leftarrow \mathbf{x} ; \lambda_{\mathrm{D}}\right) / \tilde{G}\left(\mathbf{x}_{\odot} \leftarrow \mathbf{x}_{\odot} ; \lambda_{\mathrm{D}}\right)\right\} \equiv \exp \left(-r_{\oplus}^{2} / \lambda_{\mathrm{D}}^{2}\right) .
$$

Exercise $\mathbf{n}^{0}$ 3-f - Level [2] : In this regime, work out the analytic density of probability for the reduced flux of a single clump and show that

$$
\mathscr{P}(\psi)=2 \pi \lambda_{\mathrm{D}}^{3} \frac{\rho_{\odot}}{N_{H} M_{c}} \frac{\sqrt{-\ln \psi}}{\psi} .
$$

Relation (3.51) suggests that the individual probability distribution $\mathscr{P}_{1}(\psi) \equiv \mathscr{P}(\psi)$ is strongly peaked for small values of the signal $\psi$. As a result, the probability distribution for the total flux $\psi_{\text {tot }} \equiv \Phi_{\text {rand }} / \varphi_{\text {max }}$ generated by the $N \equiv N_{H}$ subhalos of domain $\mathscr{D}_{H}$ can be approximated by

$$
\mathscr{P}_{N}\left(\psi_{\text {tot }}\right)=N \times \mathscr{P}_{1}\left(\psi_{\text {tot }}\right)
$$

as long as $0<\psi_{\text {tot }}<1$ and in the regime where $\left\langle\psi_{\text {tot }}\right\rangle$ is vanishingly small. The proof is straightforward. The probability $\mathscr{P}_{N}$ is given by the product of convolution

$$
\mathscr{P}_{N}\left(\psi_{\text {tot }}\right)=\int_{0}^{1} \mathscr{P}_{1}(\psi) \times \mathscr{P}_{N-1}\left(\psi_{\text {tot }}-\psi\right) \times d \psi
$$

When $\mathscr{P}_{N-1}\left(\psi_{\text {tot }}\right)$ behaves qualitatively like $\mathscr{P}_{1}(\psi)$ and is also strongly peaked at a value close to 0 , two regions dominate the contribution to the previous integral when $\psi_{\text {tot }}$ is less than 1 , namely $\psi$ close to 0 where $\mathscr{P}_{1}(\psi)$ is large, and $\psi$ close to $\psi_{\text {tot }}$ where $\mathscr{P}_{N-1}\left(\psi_{\text {tot }}-\psi\right)$ is large, so that

$$
\mathscr{P}_{N}\left(\psi_{\text {tot }}\right) \approx \mathscr{P}_{N-1}\left(\psi_{\text {tot }}\right)+\mathscr{P}_{1}\left(\psi_{\text {tot }}\right) \text {. }
$$

If the same operation is repeated until we are only left with the single clump distribution $\mathscr{P}_{1}(\psi)$, relation (3.52) is proved.

In figure 31, a hundred thousand realizations of the same clumpy DM halo as before have been simulated with now a substructure mass of $10^{7} M_{\odot}$. On the horizontal axis, the histogram features the boost ratio $\eta \equiv B / B_{\text {eff }}$ which is proportional to $\psi_{\text {tot }}$. The red curve which is superimposed on the Monte Carlo results corresponds to the product

$$
N_{H} \mathscr{P}_{1}(\varphi) d \varphi \equiv \frac{f}{M_{c}} \int_{\mathscr{D}_{\varphi}} \rho_{\text {smth }}(\mathbf{x}) d^{3} \mathbf{x}
$$

with the same values of $f$ and $M_{c}$ as in the simulation. On a large portion of the range extending from $\sim 0$ up to $B \sim 11 B_{\text {eff }}$, therefore for a total flux $\Phi_{\text {rand }}$ smaller than $\varphi_{\max }$, relation (3.52) is a quite good approximation. This regime corresponds to the situation where a single clump happens to contribute significantly more than the others. Most of the realizations of figure 31 correspond to small values of the flux ratio $\Phi_{\text {rand }} / \varphi_{\text {max }}$. Remember that the number of clumps effectively implied in the signal is on average very small. 


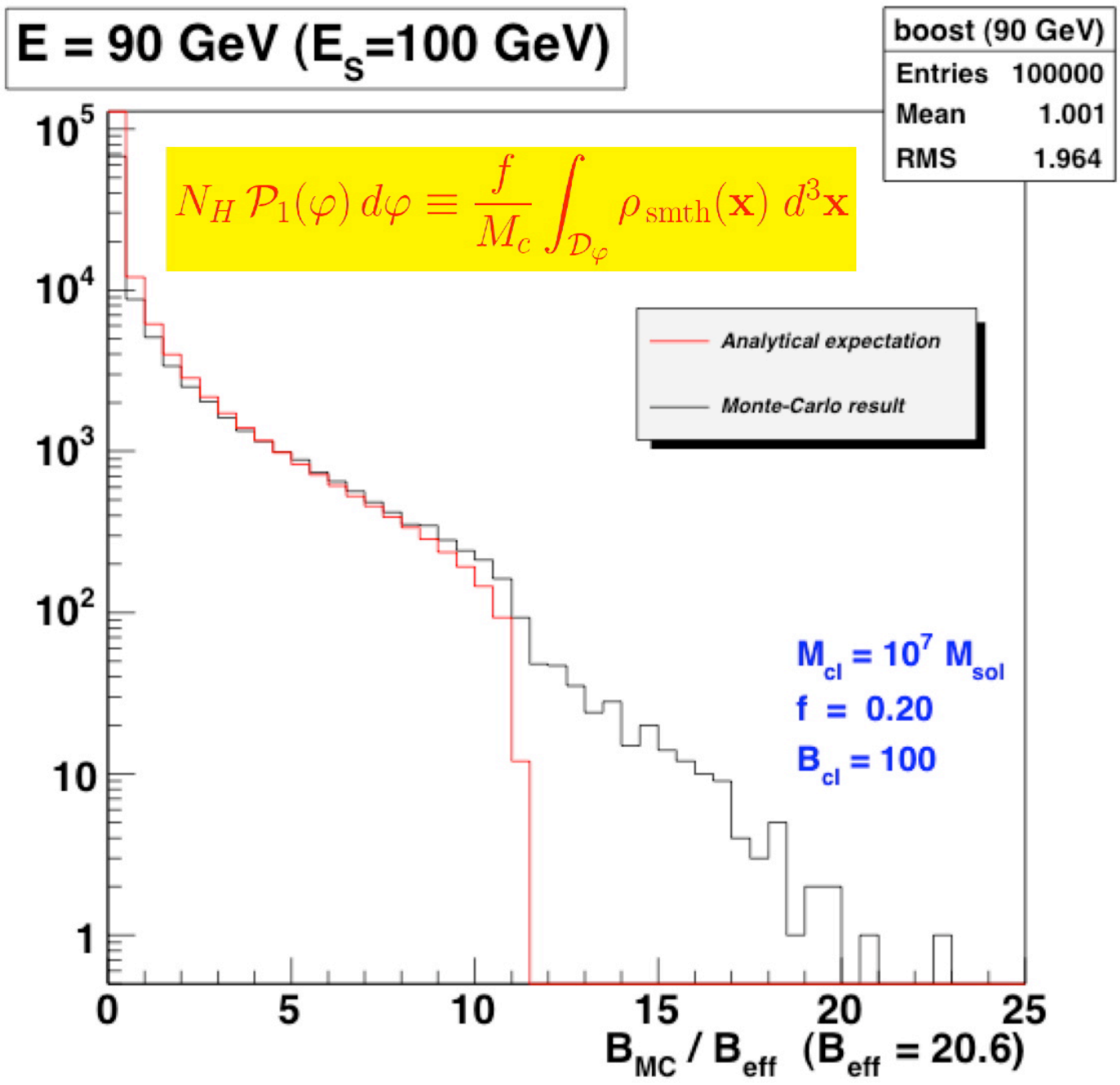

Figure 31: Same Monte Carlo simulation as before but with a clump mass of $10^{7} M_{\odot}$. Each simulated halo has a hundred times less substructures than in figure 30 and $10^{5}$ realizations have been generated. Their distribution is plotted as a function of the boost ratio $\eta=B / B$ eff and corresponds to the black histogram. The red line features the product of the total number $N_{H}$ of subhalos lying inside the Milky Way halo $\mathscr{D}_{H}$ by the single clump probability distribution $\mathscr{P}_{1}(\varphi) \equiv \mathscr{P}(\varphi)$. Figure from [87].

\subsection{Dark matter mini-spikes around black holes}

Recenty, a new scenario has been discussed in the literature, where the formation of intermediate mass black holes, i.e. black holes with mass $M$ in the range $10^{2} \lesssim M / M_{\odot} \lesssim 10^{6}$, leads to the formation of DM overdensities called mini-spikes, which might be observed as point sources of gamma rays [134] and neutrinos [135]. Although somewhat speculative, the scenario has the undisputed virtue of making specific predictions on the number and luminosity of these objects, that could be observed or ruled out in the near future with the upcoming generation of space and ground based experiments. The statistical analysis discussed in the previous section has been applied [136] to these DM mini-spikes in the case of antiprotons and positrons. Antimatter fluxes are dramatically enhanced. Typical boost factors $B_{\text {eff }} \sim 10^{4}$ bring them within the reach of current and 
upcoming experiments.

Intermediate mass black holes (hereafter IMBH) span the gap between stellar mass black holes whose mass does not exceed $\sim 100 M_{\odot}$ and super massive objects with mass larger than $10^{6} M_{\odot}$. The discovery of extremely bright X-ray sources [137] suggest that IMBHs do exist. They are naturally invoked from a theoretical point of view as the building blocks of the super massive black holes believed to power the active nuclei of many galaxies. In one of the scenarios proposed in [134], the formation of black holes with typical mass $\sim 10^{5} M_{\odot}$ is associated to the virialization of the first DM substructures. The gas which they contain cools, collapses and may form pressure-supported discs at the centers of halos that are massive enough to contain a large amount of molecular hydrogen. In halos which do not experience any major merger over a dynamical time, a protogalactic disc forms and can evolve uninterrupted. At this stage, an effective viscosity due to local gravitational instabilities in the disc leads to an inward mass transfer and outward angular momentum transfer, until supernovae in the first generation of stars heat the disc and terminate this process. By the time the process ends, a baryonic mass of order $\sim 10^{5} M_{\odot}$ loses its angular momentum and is transferred to the center of the halo, leading to the formation of an object that may be briefly pressure-supported, but which eventually collapses to form a black hole. If the latter forms at the exact center of the DM distribution - which is believed to be already highly concentrated at the core of the embedding halo - dark matter drifts inward and is further compressed. The effect of the formation of a central object on the surrounding distribution of matter has been investigated for the first time in the framework of DM annihilations in [138]. It was shown that the adiabatic growth of a massive object at the center of a cusp, i.e. a power-law radial distribution with index $\gamma$

$$
\rho_{i}^{\mathrm{h}} \propto r_{i}^{-\gamma},
$$

induces a redistribution of matter into a new power-law called spike

$$
\rho_{f}^{\mathrm{h}} \propto r_{f}^{-A}
$$

with an index $A>\gamma$.

Exercise $\mathbf{n}^{0}$ 3-g - Level [1] : In order to relate simply the indices $\gamma$ and A, we will assume that the DM species move only along circular orbits around the center $O$ of the initial cusp $\rho_{i}^{\mathrm{h}}$. The angular momentum with respect to $O$ of some particle $P$ with unit mass and velocity $\mathbf{v}$ is defined as

$$
\mathbf{L}=\mathbf{r} \wedge \mathbf{v}
$$

where $\mathbf{r}$ denotes the vector pointing from $O$ to $P$. The gravitational field experienced by the orbiting WIMPs is always directed toward the center of symmetry $O$ of the matter distribution. Show that the angular momentum $\mathbf{L}$ remains constant however rapidly the black hole forms.

Should the collapse of the central object be sudden, a WIMP with an initial circular orbit with radius $r_{i}$ would undergo a strong dynamical impulse and would be forced to migrate on a very excentric trajectory. In spite of the conservation of its angular momentum $\mathbf{L}$, the particle would enventually orbit on an elongated trajectory. If, on the contrary, the black hole forms very slowly 
- on a much longer timescale than the typical orbital period of the surrounding matter - our test WIMP keeps on rotating on a circle whose radius gradually decreases until it reaches the final value of $r_{f}$.

Exercise $\mathbf{n}^{0}$ 3-h - Level [1] : Show that the conservation of the angular momentum of the WIMP leads to

$$
r_{i} M_{i}\left(r_{i}\right)=r_{f} M_{f}\left(r_{f}\right) .
$$

The total mass which the DM particle feels at a distance $r$ from the center $O$ is denoted by $M(r)$. Infer that

$$
r_{i} M_{i}^{\mathrm{h}}=r_{f} M_{\mathrm{BH}} .
$$

The mass $M_{i}^{\mathrm{h}}$ corresponds to the DM species which initially orbit within a distance $r_{i}$ from the center $O$ and build up the cusp (3.56). After the collapse has taken place, the mass within the radius $r_{f}$ is dominated by the central black hole. Establish then that $r_{i}$ and $r_{f}$ are related by

$$
r_{f} \propto r_{i}^{4-\gamma} .
$$

The DM sphere around which our test particle orbits undergoes a contraction as a result of the collapse of the black hole at its center and its radius decreases from the initial value $r_{i}$ down to $r_{f}$. The DM species do follow that sphere which contains always the same amount of dark matter during the compression process.

Exercise $\mathbf{n}^{0}$ 3-i - Level [1] : Translating that condition into

$$
M_{i}^{\mathrm{h}}\left(r_{i}\right)=M_{f}^{\mathrm{h}}\left(r_{f}\right),
$$

show that

$$
r_{i}^{3-\gamma} \propto r_{f}^{3-A} .
$$

As in the very pedagogical analysis by [139] which has inspired these exercises, use the conditions relating $r_{i}$ and $r_{f}$ to establish that

$$
A=\frac{9-2 \gamma}{4-\gamma}
$$

The adiabatic compression of an initial DM cloud requires very specific conditions which may not be necessarily satisfied. The black hole needs to form very slowly. It must also collapse at the exact center of the initial DM distribution in order to drag it inward. We will assume hereafter that both conditions are met in some of the first DM halos whose virial mass is $\sim 10^{8} M_{\odot}$ at the time of the IMBH collapse. One should keep in mind that adiabatic spikes are indeed rather fragile structures which require fine-tuned conditions to form at the centers of galactic halos [139]. They can be easily destroyed by dynamical processes such as major mergers [140] and gravitational scattering off stars [141, 142]. 


\section{Adiabatic DM compression around the IMBH
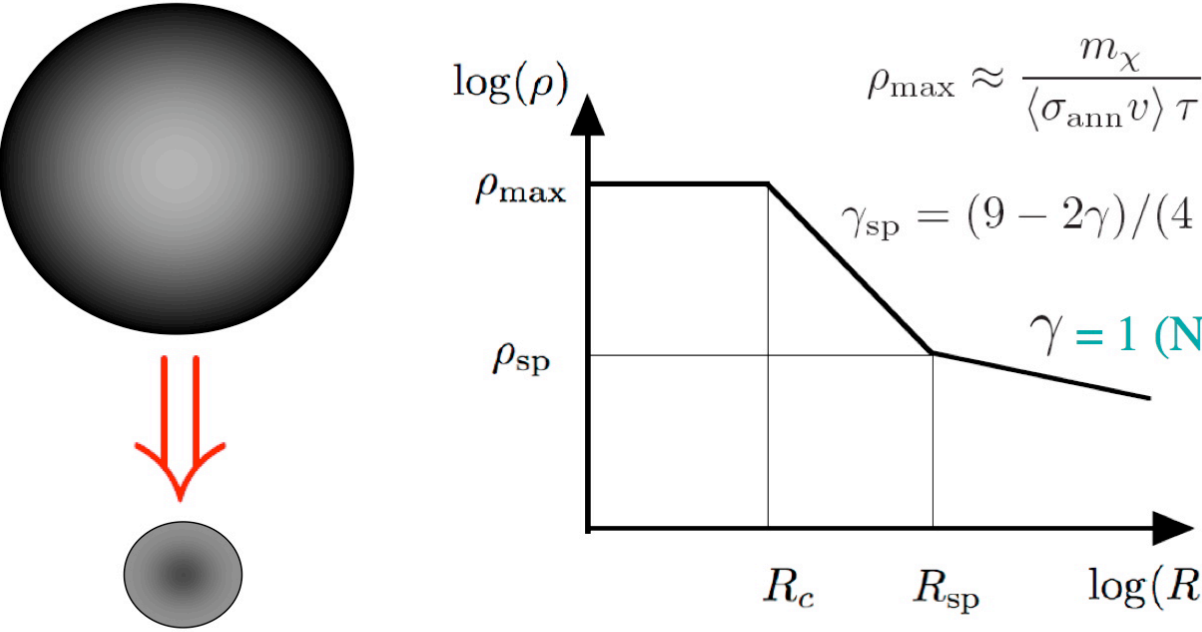 \\ Large annihilation volume

$$
\begin{gathered}
\xi=\frac{12}{5} \pi R_{\mathrm{sp}}^{3}\left\{\frac{\rho_{\mathrm{sp}}}{\rho_{\odot}}\right\}^{2}\left\{\frac{14}{9} \eta^{5 / 7}-1\right\} \text { where } \frac{R_{\mathrm{sp}}}{R_{\mathrm{c}}}=\left\{\eta \equiv \frac{\rho_{\mathrm{max}}}{\rho_{\mathrm{sp}}}\right\}^{3 / 7} \\
\xi \sim 3.3 \times 10^{6} \mathrm{kpc}^{3} \text { for }\left\langle\sigma_{\mathrm{ann}} v\right\rangle_{26}=3 \& m_{\chi}=1 \mathrm{TeV}
\end{gathered}
$$

Figure 32: Radial section of a typical DM mini-spike around its central IMBH. Inside the core of radius $R_{\mathrm{c}}$, the WIMP annihilation rate is limited by the age $\tau$ of the object. The mantle refers to the region extending from $R_{\mathrm{c}}$ to $R_{\mathrm{sp}}$ where the DM cloud has been dragged inward by the collapsing black hole. Adiabatic compression does not act beyond a radius $r=R_{\mathrm{sp}}$ where we find the pristine DM distribution of the initial surrounding halo. Figure from [136].

In the case where the DM profile before the formation of the IMBH follows the commonly adopted NFW distribution [79], the final DM density around the central object will be described by a power law $\rho(r) \propto r^{-7 / 3}$ within a region of size $R_{\mathrm{sp}}$. The inner structure of a typical mini-spike is featured in the schematic representation of figure 32. At larger distances, the DM distribution has not been modified by the accretion onto the black hole and the density still falls down as $r^{-1}$. This envelope does not contribute significantly to the DM annihilations and its associated production of positrons and antiprotons. On the contrary, the DM density steeply increases below $R_{\mathrm{sp}}$ and annihilations themselves set an upper limit to it

$$
\rho_{\max } \approx \frac{m_{\chi}}{\left\langle\sigma_{\mathrm{ann}} v\right\rangle \tau},
$$

where $\tau$ is the time elapsed since the formation of the mini-spike. The cutoff radius below which the mini-spike core extends with uniform density $\rho_{\max }$ is denoted by $R_{\mathrm{c}}$. For a typical value of $\tau=10 \mathrm{Gyr}$, we find

$$
\rho_{\max }=8.752 \times 10^{15} M_{\odot} \mathrm{kpc}^{-3} \times\left\{\frac{m_{\chi}}{1 \mathrm{GeV}}\right\} \times\left\{\frac{\left\langle\sigma_{\mathrm{ann}} v\right\rangle}{10^{-26} \mathrm{~cm}^{3} \mathrm{~s}^{-1}}\right\}^{-1} .
$$


The relative extension of the mini-spike mantle $\left(R_{\mathrm{c}} \leq r \leq R_{\mathrm{sp}}\right)$ with respect to the core $\left(r \leq R_{\mathrm{c}}\right)$ is given by

$$
\frac{R_{\mathrm{sp}}}{R_{\mathrm{c}}}=\left\{\eta \equiv \frac{\rho_{\mathrm{max}}}{\rho_{\mathrm{sp}}}\right\}^{3 / 7},
$$

where $\rho_{\mathrm{sp}}=\rho\left(R_{\mathrm{sp}}\right)$ is the DM density at the surface $r=R_{\mathrm{sp}}$ of the mantle.

Exercise $\mathbf{n}^{0} \mathbf{3}-\mathbf{j}$ - Level [1] : Integrate equation (3.10) over the inner structure of a mini-spike and establish that the annihilation volume is given by

$$
\xi=\frac{12}{5} \pi R_{\mathrm{sp}}^{3}\left\{\frac{\rho_{\mathrm{sp}}}{\rho_{\odot}}\right\}^{2}\left\{\frac{14}{9} \eta^{5 / 7}-1\right\} .
$$

On average, the mini-spike radius is equal to $R_{\mathrm{sp}}=2.84 \mathrm{pc}$ with a density $\rho_{\mathrm{sp}}$ of $48.51 \mathrm{M}_{\odot} p \mathrm{c}^{-3}$. Assuming a benchmark cross section $\left\langle\sigma_{\mathrm{ann}} v\right\rangle$ of $3 \times 10^{-26} \mathrm{~cm}^{3} \mathrm{~s}^{-1}$ and a WIMP mass $m_{\chi}$ of $1 \mathrm{TeV}$, show that the annihilation volume $\xi$ reaches a value of $\sim 3.3 \times 10^{6} \mathrm{kpc}^{3}$. The solar neighborhood DM density $\rho_{\odot}$ is set equal to the canonical value of $0.3 \mathrm{GeV} \mathrm{cm}^{-3}$

Should we know the exact location and annihilation volume of each mini-spike, we would unambiguously derive the positron and antiproton cosmic ray fluxes at the Earth. This is not the case. These signals are actually affected by some sort of galactic variance whose analysis requires the statistical tools developed in section 3.1. A representative set of different halo realizations including mini-spikes has been built in [136]. Monte Carlo simulations have been performed on the basis of the $\sim 200$ different realizations of the mini-spike population which have been obtained in [134] by evolving an initial distribution of IMBHs orbiting in the Milky Way halo and by allowing the associated DM minihalos to be tidally destroyed during close encounters. The distribution of the number $N_{\mathrm{BH}}$ of surviving mini-spikes follows a Gaussian distribution with a mean value of $\sim 100$ objects within a galactocentric radius of $100 \mathrm{kpc}-$ of which $\sim 60$ populate the $\mathrm{DH}-$ and a rms $\sigma_{N}$ equal to $\sim 21$. No significant correlation was found between the position $\mathbf{x}$ and annihilation volume $\xi$ of the mini-spikes in the simulations of [134]. These characteristic quantities are respectively distributed according to the probability functions $q(\xi)$ and $p(\mathbf{x})$ specified in [136] and which allow to define the average values

$$
\left\langle\mathscr{G}^{n}\right\rangle=\int_{\mathrm{DH}} d^{3} \mathbf{x} \mathscr{G}^{n}\left(\mathbf{x}_{\odot} \leftarrow \mathbf{x}\right) p(\mathbf{x}),
$$

and

$$
\left\langle\xi^{n}\right\rangle=\int_{0}^{+\infty} \xi^{n} q(\xi) d \xi
$$

Exercise $\mathbf{n}^{0} 3-\mathbf{k}-$ Level [2] : Generalize relation (3.22) to the case where the number $N_{\mathrm{BH}}$ of mini-spikes may vary and show that

$$
\frac{\sigma_{\mathrm{rand}}^{2}}{\left\langle\Phi_{\text {rand }}\right\rangle^{2}}=\frac{1}{\left\langle N_{\mathrm{BH}}\right\rangle}\left\{\frac{\left\langle\xi^{2}\right\rangle}{\langle\xi\rangle^{2}} \frac{\left\langle\mathscr{G}^{2}\right\rangle}{\langle\mathscr{G}\rangle^{2}}-1\right\}+\frac{\sigma_{N}^{2}}{\left\langle N_{\mathrm{BH}}\right\rangle^{2}} .
$$



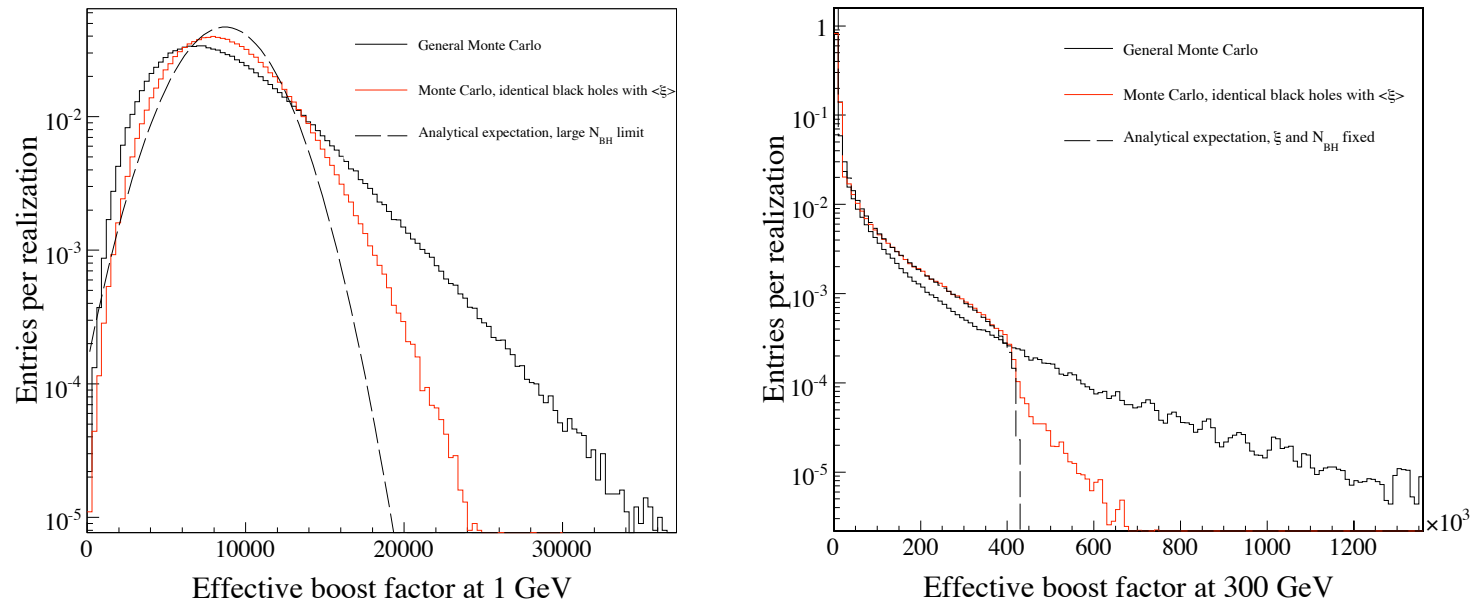

Figure 33: A $1 \mathrm{TeV}$ positron line has been assumed. The boost factor is computed with respect to the NFW distribution of table 7 with a scale radius of $20 \mathrm{kpc}$. Its distribution is featured for two values of the positron energy $E$ at the Earth. In both panels, the black solid curve features the results of the full Monte Carlo where the number $N_{\mathrm{BH}}$ of objects per realization, their positions $\mathbf{x}$ and annihilation volumes $\xi$ are chosen at random. The red solid line is obtained by setting $\xi$ to its average value. The black long-dashed curve is the theoretical expectation based on the analytical analysis of section 3.1. Figure from [136].

The pedagogical case of a $1 \mathrm{TeV}$ positron line is featured in figure 33. The distributions of the boost factor at $1 \mathrm{GeV}$ obtained with the Monte Carlo simulations of [136] are displayed in the left panel. The red histogram corresponds to the case of identical mini-spikes with fixed annihilation volume. It can be compared to the dashed curve, which is the analytical estimate of the boost factor distribution in the limiting case where an infinite number of IMBHs are present in the Milky Way. If it were so, the central limit theorem would apply and the distribution of the boost factor would be a Gaussian. This is actually almost the case for identical objects. The statistical distribution obtained in the general case is plotted in black. Having different annihilation volumes for different mini-spikes shifts the distribution away from the Gaussian behaviour. The distribution of the boost factor for positrons detected at the Earth at $300 \mathrm{GeV}$ is displayed in the right panel. Again, the red histogram corresponds to identical objects and should be compared to the black long-dashed analytical estimate. The latter includes only one IMBH inside the positron horizon at $300 \mathrm{GeV}$ so that the extra events of the red line correspond to the (very) rare situations in which two minispikes contribute to the signal. This feature is not present when the full Monte Carlo is run. It is erased by the random choice of $\xi$.

The antiproton signal has been derived in figure 34 for two realistic and very different WIMP models which both match the constraints from collider experiments and relic density. In the left panel, the case of a $140 \mathrm{GeV}$ Bino has been examined in the framework of the minimal supersymmetric standard model (MSSM) with a gravity driven supersymmetry breaking (mSUGRA). That particle is characterized by an annihilation cross section $\left\langle\sigma_{\mathrm{ann}} v\right\rangle$ of $0.26 \times 10^{-26} \mathrm{~cm}^{3} \mathrm{~s}^{-1}$ and a branching ratio of $91 \%$ into $b \bar{b}$ pairs and $9 \%$ into $\tau^{+} \tau^{-}$pairs. Relation (3.68) leads to an average annihilation volume $\langle\xi\rangle$ of $4.66 \times 10^{6} \mathrm{kpc}^{3}$. The Kaluza-Klein inspired model of [129] is presented in the right panel. It is based on warped extra-dimensions in the context of a SO(10) GUT. The DM species is 

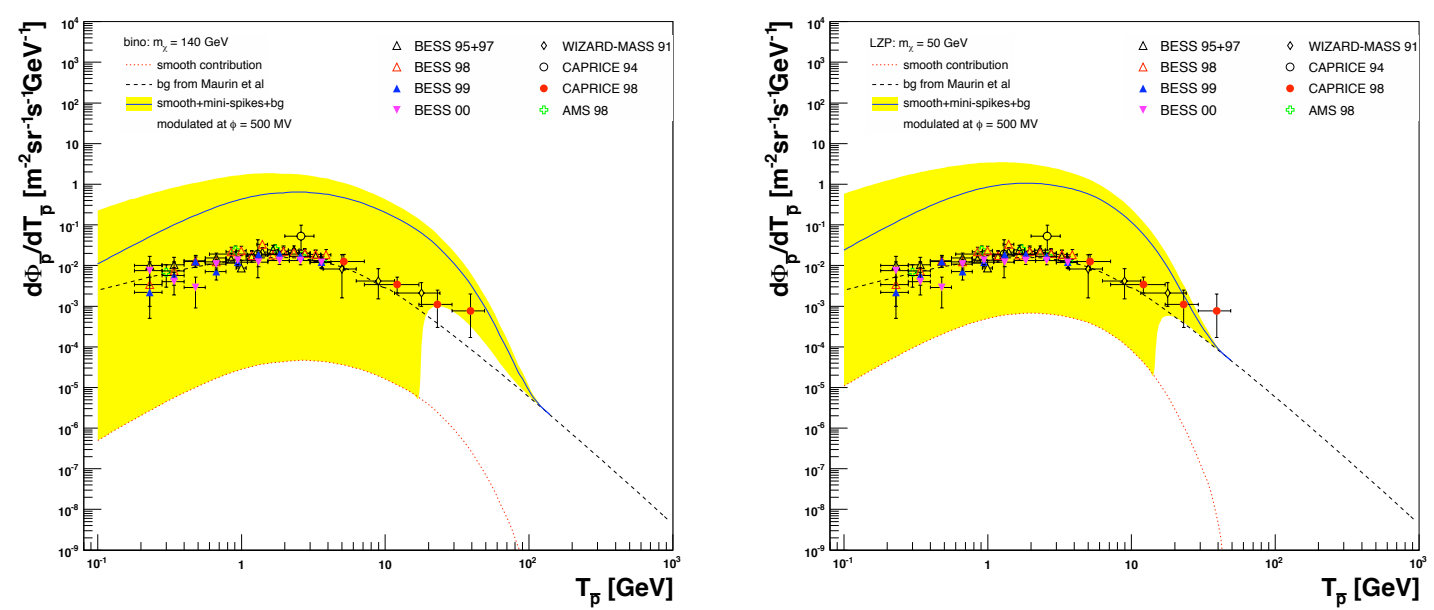

Figure 34: Antiproton fluxes as a function of kinetic energy for a $140 \mathrm{GeV}$ Bino (left panel) and a $50 \mathrm{GeV}$ LZP species [129] (right panel). The black short-dashed curve is the background of secondary antiprotons whereas the red dotted line is the signal from a completely smooth DM halo following the NFW distribution of table 7. The yellow area corresponds to the 1-sigma uncertainty band arising from galactic variance and has been derived by a Monte Carlo simulation of the mini-spike population. The blue solid curve is the average signal including the background and the contribution from the smooth DM distribution. Observations collected by various experiments $[57,58,59,60]$ are featured for comparison. Figure from [136].

a right-handed Dirac neutrino. Its stability arises from the conservation of a $\mathbf{Z}_{3}$ symmetry, hence its name LZP for lightest Z particle. Setting the Kaluza-Klein scale $M_{K K}$ at a value of $6 \mathrm{TeV}$ leads in the case of a $50 \mathrm{GeV}$ LZP to an annihilation cross section of $2.04 \times 10^{-26} \mathrm{~cm}^{3} \mathrm{~s}^{-1}$ and ensures a correct relic density. Such a value is $\sim$ an order of magnitude larger than is the Bino case. The quark channels amount to $74 \%$ of the annihilations whereas the branching ratio into neutrino pairs is $17 \%$. Charged lepton pairs $l^{+} l^{-}$are equally produced with a branching ratio of $\sim 3 \%$ each. The LZP provides thus an illustration of a WIMP with a positron line. With an average value of $5.14 \times 10^{5} \mathrm{kpc}^{3}$, the LZP annihilation volume is much smaller than for the Bino. In the case of a smooth halo, the exotic contribution (red dotted line) is always much lower than the background of secondary antiprotons (black short-dashed curve). When IMBH mini-spikes are included and their galactic population modeled with a Monte Carlo, the average antiproton flux (blue solid curve) at $10 \mathrm{GeV}$ is always $\sim$ two orders of magnitude above the background. The yellow band features the 1-sigma uncertainty of the signal arising from the variance in the galactic distribution of minispikes. Notice how similar the two panels are in spite of very different values for the cross section and the annihilation volume.

Exercise $\mathbf{n}^{0} 3$-1 - Level [2] : The individual contribution $\varphi_{i}$ of a mini-spike scales as $\mathscr{F} \times \xi_{i}$. Show that it is proportional to

$$
\varphi_{\text {mini-spike }} \propto\left\langle\sigma_{\mathrm{ann}} v\right\rangle^{2 / 7} \times m_{\chi}^{-9 / 7} .
$$

The antiproton signal depends weakly on the annihilation cross section since a decrease of the 
latter is partially compensated by a higher annihilation volume. Below $20 \mathrm{GeV}$, the mini-spike fluxes have a very large scatter because the antiproton horizon tends to shrink. As explained at the beginning of section 2.2, galactic convection is no longer negligible at low energy. The formation of mini-spikes around intermediate mass black holes generates dramatic enhancements of the DM density and of the annihilation signals at the Earth, with a typical increase of order $10^{4}$. For a typical mini-spike population, the flux of primary positrons and antiprotons turns out to be one to two orders of magnitude larger than the measured flux, and is fairly insensitive to the specific properties of the DM species. As shown above, the annihilation cross section has little influence because of the presence of an annihilation plateau in the cores of mini-spikes. As the average flux exceeds the data so far collected, we could naively conclude that the entire scenario is already ruled out by observation. However, because of the small number of objects, the variance associated to the positron and antiproton signals is enormous and no definitive conclusion can yet be reached. Further investigations are required to estimate the confidence level at which the mini-spike scenario may still be acceptable.

\subsection{The boost factor of $\Lambda-C D M$ clumps as a conclusion}

To conclude these lectures on indirect detection, I would like to mention a recent and very important piece of work [143]. Using the tools presented in section 3.1, the boost factor for positrons and antiprotons has been derived in the case of the DM substructures found in numerical simulations. A large variety of models have been considered. The mass distribution of clumps is a power law whose index $\sim 1.8$ to 2 . The low mass cutoff has been varied and values as low as $10^{-6} M_{\odot}$ have been assumed. The galactic distribution of subhalos is not well known since tidal disruption could prevent them from populating the central regions of the Milky Way. Finally, the inner DM profiles of clumps - below their scale radii - may be described either by a NFW or a Moore distribution with an index comprised between 1 and 3/2. The crucial result is that even in the best possible case, the boost factor does not exceed a value of $\sim 10$ to 20 . The antimatter cosmic ray signature of DM species cannot be enhanced very much above what a smooth NFW halo yields. Unless the annihilation cross section is increased by resonant effects - as in the case of heavy Wino-like neutralinos - we should not expect much from antiprotons and positrons. The gamma ray signature is more promising insofar as it is possible to focuse the observation on any hot spot in the sky but WIMPs need to be very concentrated though.

Acknowledgement : I would like to thank Géraldine Servant, Jean Orloff and Gérard Smadja for their very warm welcome as well as for the patience and dedication with which they have organized this exciting and inspiring Cargèse Summer School. I express to them my gratitude for their encouragement in writing these proceedings. Many thanks also to Catherine Pinty for her efficiency in dealing with the French bureaucracy. The school could not have been so well organized without the help of the Cargèse conference center - special thanks to Claudine, Vittoria and Mohamed. 


\section{References}

[1] G. Gentile, P. Salucci, U. Klein, D. Vergani and P. Kalberla, The cored distribution of dark matter in spiral galaxies, Mon. Not. Roy. Astron. Soc. 351 (2004) 903 [astro-ph/ 0403154 ].

[2] J. N. Bahcall and R. M. Soneira, The Universe at faint magnitudes. 2. Models for the predicted star counts, Astrophys. J. Suppl. 44 (1980) 73.

[3] R. Bernabei et al. (DAMA Collaboration), Dark Matter search, Riv. Nuovo Cim. 26N1 (2003) 1 [astro-ph/0307403].

[4] D. S. Akerib et al. (CDMS Collaboration), Exclusion limits on the WIMP-nucleon cross-section from the first run of the cryogenic dark matter search in the Soudan underground Lab, Phys. Rev. D72 (2005) 052009 [astro-ph/0507190].

[5] J. Angle et al. (XENON Collaboration), First results from the XENON10 dark matter experiment at the Gran Sasso National Laboratory, Phys. Rev. Lett. 100 (2008) 021303 [arXiv : 0706.0039 ].

[6] M. W. Goodman and E. Witten, Detectability of certain dark matter candidates, Phys. Rev. D31 (1985) 3059; L. M. Krauss and P. Romanelli, New signatures for dark matter detectors, Phys. Rev. D39 (1989) 1225; R. Flores, K. A. Olive and M. Srednicki, Elastic neutralino-matter scattering, Phys. Lett. B237 (1990) 72.

[7] A. Ashman et al. (European Muon Collaboration), A measurement of the spin asymmetry and determination of the structure function $g(1)$ in deep inelastic muon-proton scattering, Phys. Lett.

B206 (1988) 364; R. L. Jaffe and A. Manohar, The g(1) problem : fact and fantasy on the spin of the proton, Nucl. Phys. B337 (1990) 509.

[8] D. Adams et al. (Spin Muon Collaboration), Measurement of the spin dependent structure function gl(x) of the proton, Phys. Lett. B329 (1994) 399 [hep-ph/94 04270 ].

[9] A. Airapetian et al. (HERMES Collaboration), Precise determination of the spin structure function $g_{1}$ of the proton, deuteron and neutron, Phys. Rev. D75 (2007) 012007 [hep-ex/ 0609039 ].

[10] J. Engel, S. Pittel and P. Vogel, Nuclear physics of dark matter detection, Int. J. Mod. Phys. E1 (1992) 1 .

[11] M. T. Ressell et al., Nuclear shell model calculations of neutralino-nucleus cross sections for ${ }^{29}$ Si and ${ }^{73}$ Ge, Phys. Rev. D48 (1993) 5519.

[12] V. Dimitrov, J. Engel and S. Pittel, Scattering of weakly interacting massive particles from ${ }^{73}$ Ge, Phys. Rev. D51 (1995) 291 [hep-ph/9408246].

[13] J. Engel, M. T. Ressell, I. S. Towner and E. Ormand, Response of mica to weakly interacting massive particles, Phys. Rev. C52 (1995) 2216 [hep-ph/9504322].

[14] J. Engel, S. Pittel, E. Ormand and P. Vogel, Scattering of neutralinos from niobium, Phys. Lett. B275 (1992) 119.

[15] F. Iachello, L. M. Krauss and G. Maino, Spin dependent scattering of weakly interacting massive particles in heavy nuclei, Phys. Lett. B254 (1991) 220.

[16] G. Jungman, M. Kamionkowski and K. Griest, Supersymmetric dark matter, Phys. Rept. 267 (1996) 195 [hep-ph/9506380].

[17] A. Bottino, F. Donato, N. Fornengo and S. Scopel, Implications for relic neutralinos of the theoretical uncertainties in the neutralino-nucleon cross section, Astropart. Phys. 13 (2000) 215

[hep-ph/9909228]. 
[18] J. Gasser, H. Leutwyler and M. E. Sainio, Form-factor of the sigma term, Phys. Lett. B253 (1991) 260.

[19] J. Gasser and H. Leutwyler, Quark masses, Phys. Rept. 87 (1982) 77.

[20] T. P. Cheng, Chiral symmetry and the Higgs nucleon coupling, Phys. Rev. D38 (1988) 2869.

[21] B. Borasoy and Ulf-G. Meissner, Chiral expansion of baryon masses and sigma-terms, Annals Phys. 254 (1997) 192 [hep-ph/9607432].

[22] S. Weinberg, The problem of mass, Trans. New York Acad. Sci. 38 (1977) 185.

[23] J. Bijnens, J. Prades and E. de Rafael, Light quark masses in QCD, Phys. Lett. B348 (1995) 226 [hep-ph/9411285].

[24] T. Hatsuda and T. Kunihiro, Strange quark, heavy quarks, and gluon contents of light hadrons, Nucl. Phys. B387 (1992) 715.

[25] J. Gasser, H. Leutwyler and M. E. Sainio, Sigma term update, Phys. Lett. B253 (1991) 252.

[26] H.-Y. Cheng, Low-energy interactions of scalar and pseudoscalar Higgs bosons with baryons, Phys. Lett. B219 (1989) 347.

[27] M. A. Shifman, A. I. Vainshtein and V. I. Zakharov, Remarks on Higgs boson interactions with nucleons, Phys. Lett. B78 (1978) 443.

[28] S. P. Ahlen et al., Limits on cold dark matter candidates from an ultralow background germanium spectrometer, Phys. Lett. B195 (1987) 603.

[29] K. Freese, J. A. Frieman and A. Gould, Signal modulation in cold dark matter detection, Phys. Rev. D37 (1988) 3388.

[30] R. H. Helm, Phys. Rev. 104 (1956) 1466.

[31] J. Engel, Nuclear form-factors for the scattering of weakly interacting massive particles, Phys. Lett. B264 (1991) 114.

[32] D. S. Akerib et al. (CDMS Collaboration), Limits on spin-independent WIMP-nucleon interactions from the two-tower run of the cryogenic dark matter search, Phys. Rev. Lett. 96 (2006) 011302 [astro-ph/0509259].

[33] B. Sadoulet; Dark matter : at the brink of a discovery, talk given at the conference Energetic events in the universe : from physics to cosmology June 25-28 2007, Marseille, France.

[34] The CDMS - Cryogenic Dark Matter Search - homepage can be found at http://cdms.berkeley.edu/.

[35] The Edelweiss - Expérience pour DEtecter Les WIMPs En Site Souterrain - homepage can be found at http://edelweiss.in2p3.fr/.

[36] The Soudan Underground Laboratory homepage can be found at http: //www.hep.umn.edu/soudan/brochure.html.

[37] The French Laboratoire Souterrain de Modane (LSM) homepage can be found at http: //www-1sm.in2p3.fr/.

[38] The Laboratori Nazionali del Gran Sasso (LNGS) homepage can be found at http://www.lngs.infn.it/.

[39] B. Neganov and V. Trofimov, Otkrytia Izobreteniya 146 (1985) 215. 
[40] P. N. Luke, Voltage-assisted calorimetric ionization detector, Journal of Applied Physics 64 (1988) 6858.

[41] D. S. Akerib et al. (CDMS Collaboration), First results from the cryogenic dark matter search in the Soudan underground Lab, Phys. Rev. Lett. 93 (2004) 211301 [astro-ph / 0405033 ].

[42] The XENON dark matter search experiment homepage can be found at http://xenon.astro.columbia.edu/.

[43] E. Aprile; The XENON dark matter search : status of the XENON10 phase, talk given at IXth international conference on Topics in Astroparticle and Underground Physics TAUP 2005 September 10-14, Zaragoza, Spain.

[44] The web site of the interactive plotter for experimental and theoretical data on WIMP direct detection can be found at http: //dendera.berkeley.edu/plotter/entryform.html.

[45] The homepage of the ILIAS working group in charge of background simulations can be found at http://ilias-darkmatter.uni-tuebingen.de/BSNS_WG.html.

[46] S. Yellin, Finding an upper limit in the presence of unknown background, Phys. Rev. D66 (2002) 032005 [physics/0203002].

[47] B. Morgan, A. Green and N. Spooner, Directional statistics for WIMP direct detection, Phys. Rev. D71 (2005) 103507 [astro-ph/ 0408047 ].

[48] F. Casse, M. Lemoine and G. Pelletier, Transport of cosmic rays in chaotic magnetic fields, Phys. Rev. D65 (2002) 023002 [astro-ph/0109223].

[49] D. Maurin, R. Taillet, F. Donato, P. Salati, A. Barrau and G. Boudoul, Galactic cosmic ray nuclei as a tool for astroparticle physics, [astro-ph/0212111].

[50] D. Maurin, F. Donato, R. Taillet and P. Salati, Cosmic rays below $Z=30$ in a diffusion model : new constraints on propagation parameters, Astrophys. J. 555 (2001) 585 [astro-ph/ 0101231 ].

[51] F. Donato, D. Maurin, P. Salati, R. Taillet, A. Barrau and G. Boudoul, Antiprotons from spallations of cosmic rays on interstellar matter, Astrophys. J. 563 (2001) 172 [astro-ph/0103150].

[52] F. Donato, N. Fornengo, D. Maurin, P. Salati and R. Taillet, Antiprotons in cosmic rays from neutralino annihilation, Phys. Rev. D69 (2004) 063501 [astro-ph/ 0306207 ].

[53] T. Bringmann and P. Salati, Galactic antiproton spectrum at high energies : background expectation versus exotic contributions, Phys. Rev. D75 (2007) 083006 [astro-ph / 0612514 ].

[54] D. Maurin, R. Taillet and C. Combet, Transport of exotic anti-nuclei : I-Fast formulae for antiproton fluxes, [astro-ph/0609522].

[55] L. C. Tan and L. K. Ng, Parameterization of anti-P invariant cross-section in P P collisions using a new scaling variable, Phys. Rev. D26 (1982) 1179.

[56] L. C. Tan and L. K. Ng, Calculation of the equilibrium anti-proton spectrum, J. Phys. G9 (1983) 227.

[57] S. Orito et al. (BESS Collaboration), Precision measurement of cosmic ray antiproton spectrum, Phys. Rev. Lett. 84 (2000) 1078 [astro-ph/9906426].

[58] T. Maeno et al. (BESS Collaboration), Successive measurements of cosmic ray antiproton spectrum in a positive phase of the solar cycle, Astropart. Phys. 16 (2001) 121 [astro-ph/0010381].

[59] M. Boezio et al. (WiZard/CAPRICE Collaboration), The cosmic ray anti-proton flux between 3-GeV and 49-GeV, Astrophys. J. 561 (2001) 787 [astro-ph/0103513]. 
[60] M. Aguilar et al. (AMS Collaboration), The Alpha magnetic spectrometer (AMS) on the international space station. I: Results from the test flight on the space shuttle, Phys. Rept. 366 (2002) 331, Erratum-ibid. 380 (2003) 97.

[61] J. S. Perko, Solar modulation of galactic antiprotons, Astron. \& Astrophys. 184 (1987) 119.

[62] L. Bergström, J. Edsjö and P. Ullio, Cosmic anti-protons as a probe for supersymmetric dark matter?, Astrophys. J. 526 (1999) 215 [astro-ph / 9902012 ].

[63] T. Sanuki et al. (BESS Collaboration), Precise measurement of cosmic ray proton and helium spectra with the BESS spectrometer, Astrophys. J. 545 (2000) 1135 [astro-ph/ 0002481 ].

[64] J. Alcaraz et al. (AMS Collaboration), Protons in near Earth orbit, Phys. Lett. B472 (2000) 215 [hep-ex/ 0002049 ] - Cosmic protons, Phys. Lett. B490 (2000) 27 - Helium in near Earth orbit, Phys. Lett. B494 (2000) 193.

[65] A. M. Lionetto, A. Morselli and V. Zdravkovic, Uncertainties of cosmic ray spectra and detectability of antiproton mSUGRA contributions with PAMELA, JCAP 0509 (2005) 010 [astro-ph/0502406].

[66] A. W. Strong and I. V. Moskalenko, Propagation of cosmic ray nucleons in the Galaxy, Astrophys. J. 509 (1998) 212 [astro-ph/9807150].

[67] J. Hisano, S. Matsumoto and M. M. Nojiri, Explosive dark matter annihilation, Phys. Rev. Lett. 92 (2004) 031303 [hep-ph/0307216]; J. Hisano, S. Matsumoto, M. M. Nojiri and O. Saito, Non-perturbative effect on dark matter annihilation and gamma ray signature from galactic center, Phys. Rev. D71 (2005) 063528 [hep-ph/ 0412403 ].

[68] T. Appelquist, H. C. Cheng and B. A. Dobrescu, Bounds on universal extra dimensions, Phys. Rev. D64 (2001) 035002 [hep-ph/ 0012100$].$

[69] T. Bringmann, High-energetic cosmic antiprotons from Kaluza-Klein dark matter, JCAP 0508 (2005) 006 [astro-ph/0506219].

[70] A. Barrau, P. Salati, G. Servant, F. Donato, J. Grain, D. Maurin and R. Taillet, Kaluza-Klein dark matter and galactic antiprotons, Phys. Rev. D72 (2005) 063507 [astro-ph / 0506389 ].

[71] H. Baer, T. Krupovnickas, S. Profumo and P. Ullio, Model independent approach to focus point supersymmetry : from dark matter to collider searches, JHEP 0510 (2005) 020 [hep-ph/0507282]; U. Chattopadhyay, D. Choudhury, M. Drees, P. Konar and D. P. Roy, Looking for a heavy Higgsino LSP in collider and dark matter experiments, Phys. Lett. B632 (2006) 114 [hep-ph/0508098] and references therein.

[72] S. Profumo and C. E. Yaguna, A statistical analysis of supersymmetric dark matter in the MSSM after WMAP, Phys. Rev. D70 (2004) 095004 [hep-ph / 0407036$].$

[73] P. Ullio, Indirect detection of neutralino dark matter candidates in anomaly-mediated supersymmetry breaking scenarios, JHEP 0106 (2001) 053 [hep-ph / 0105052 ].

[74] L. Bergström, T. Bringmann, M. Eriksson and M. Gustafsson, Gamma rays from heavy neutralino dark matter, Phys. Rev. Lett. 95 (2005) 241301 [hep-ph / 0507229 ].

[75] U. Chattopadhyay, D. Das, P. Konar and D. P. Roy, Looking for a heavy Wino LSP in collider and dark matter experiments, Phys. Rev. D75 (2007) 073014 [hep-ph / 0610077 ]. 
[76] H. C. Cheng, K. T. Matchev and M. Schmaltz, Radiative corrections to Kaluza-Klein masses, Phys. Rev. D66 (2002) 036005 [hep-ph/ 0204342 ]; G. Servant and T. M. P. Tait, Is the lightest Kaluza-Klein particle a viable dark matter candidate?, Nucl. Phys. B650 (2003) 391 [hep-ph/0206071].

[77] M. Kakizaki, S. Matsumoto and M. Senami, Relic abundance of dark matter in the minimal universal extra dimension model, Phys. Rev. D74 (2006) 023504 [hep-ph / 0605280 ].

[78] K. Kong and K. T. Matchev, Precise calculation of the relic density of Kaluza-Klein dark matter in universal extra dimensions, JHEP 0601 (2006) 038 [hep-ph / 0509119 ].

[79] J. F. Navarro, C. S. Frenk and S. D. M. White, A universal density profile from hierarchical clustering, Astrophys. J. 490 (1997) 493 [astro-ph/9611107].

[80] J. Diemand, B. Moore and J. Stadel, Convergence and scatter of cluster density profiles, Mon. Not. Roy. Astron. Soc. 353 (2004) 624 [astro-ph/ 0402267 ].

[81] B. Moore, S. Ghigna, F. Governato, G. Lake, T. Quinn, J. Stadel and P. Tozzi, Dark matter substructure within galactic halos, Astrophys. J. 524 (1999) L19.

[82] V. R. Eke, J. F. Navarro and M. Steinmetz, The power spectrum dependence of dark matter halo concentrations, Astrophys. J. 554 (2001) 114 [astro-ph/ 0012337 ].

[83] N. Fornengo, L. Pieri and S. Scopel, Neutralino annihilation into gamma rays in the Milky Way and in external galaxies, Phys. Rev. D70 (2004) 103529 [hep-ph/ 0407342 ].

[84] V. S. Berezinsky, A. V. Gurevich and K. P. Zybin, Distribution of dark matter in the galaxy and the lower limits for the masses of supersymmetric particles, Phys. Lett. B294 (1992) 221.

[85] J. Hisano, S. Matsumoto, O. Saito and M. Senami, Heavy Wino-like neutralino dark matter annihilation into antiparticles, Phys. Rev. D73 (2006) 055004 [hep-ph / 0511118 ].

[86] E. A. Baltz and J. Edsjö, Positron propagation and fluxes from neutralino annihilation in the halo, Phys. Rev. D59 (1999) 023511 [astro-ph / 9808243 ].

[87] J. Lavalle, J. Pochon, P. Salati and R. Taillet, Clumpiness of dark matter and the positron annihilation signal, Astron. \& Astrophys. 462 (2007) 827 [astro-ph/ 0603796 ].

[88] T. Delahaye, R. Lineros, F. Donato, N. Fornengo and P. Salati, Positrons from dark matter annihilation in the galactic halo : theoretical uncertainties, to be published in Phys. Rev. D (2008) [arXiv:0712.2312].

[89] I. V. Moskalenko and A. W. Strong, Production and propagation of cosmic ray positrons and electrons, Astrophys. J. 493 (1998) 694 [astro-ph/9710124].

[90] F. W. Stecker, The cosmic $\gamma$-ray spectrum from secondary particle production in cosmic ray interactions, Astrophys. \& Space Sc. 6 (1970) 377.

[91] G. D. Badhwar, S. A. Stephens and R. L. Golden, Analytic representation of the proton-proton and proton-nucleus cross sections and its application to the sea-level spectrum and charge ratio of muons, Phys. Rev. D15 (1977) 820.

[92] L. C. Tan and L. K. Ng, Parametrisation of hadron inclusive cross sections in $p-p$ collisions extended to very low energies, J. Phys. G9 (1983) 1289.

[93] T. Kamae, N. Karlsson, T. Mizuno, T. Abe and T. Koi, Parameterization of $\gamma, e^{ \pm}$and neutrino spectra produced by p-p interaction in astronomical environment, Astrophys. J. 647 (2006) 692 [astro-ph/0605581]. 
[94] Y. Shikaze et al. (BESS Collaboration), Measurements of 0.2 to $20 \mathrm{GeV/n}$ cosmic ray proton and helium spectra from 1997 through 2002 with the BESS spectrometer, Astropart. Phys. 28 (2007) 154 [astro-ph/0611388].

[95] M. Boezio et al. (WiZard/CAPRICE Collaboration), The cosmic ray electron and positron spectra measured at 1 AU during solar minimum activity, Astrophys. J. 532 (2000) 653.

[96] M. A. Duvernois et al. (HEAT Collaboration), Cosmic-ray electrons and positrons from 1 to 100 GeV : measurements with HEAT and their interpretation, Astrophys. J. 559 (2001) 296.

[97] S. W. Barwick et al. (HEAT Collaboration), Measurements of the cosmic ray positron fraction from 1-GeV to 50-GeV, Astrophys. J. 482 (1997) L191 [astro-ph/9703192].

[98] D. Hooper and J. Silk, Searching for dark matter with future cosmic positron experiments, Phys. Rev. D71 (2005) 083503 [hep-ph/ 0409104 ].

[99] J. Alcaraz et al. (AMS Collaboration), Leptons in near Earth orbit, Phys. Lett. B484 (2000) 10.

[100] M. Aguilar et al. (AMS-01 Collaboration), Cosmic-ray positron fraction measurement from $1 \mathrm{GeV}$ to $30 \mathrm{GeV}$ with AMS-01, Phys. Lett. B646 (2007) 145 [astro-ph/ 0703154 ].

[101] C. Grimani et al. (MASS Collaboration), Measurements of the absolute energy spectra of cosmic ray positrons and electrons above 7 GeV, Astron. \& Astrophys. 392 (2002) 287.

[102] J. Hisano, S. Matsumoto and M. M. Nojiri, Unitarity and higher-order corrections in neutralino dark matter annihilation into two photons, Phys. Rev. D67 (2003) 075014 [hep-ph/ 0212022 ].

[103] L. Bergström, Radiative processes in dark matter photino annihilation, Phys. Lett. B225 (1989) 372.

[104] J. F. Beacom, N. F. Bell and G. Bertone, Gamma ray constraint on galactic positron production by MeV dark matter, Phys. Rev. Lett. 94 (2005) 171301 [astro-ph / 0409403 ].

[105] L. Bergström, T. Bringmann, M. Eriksson and M. Gustafsson, Gamma rays from Kaluza-Klein dark matter, Phys. Rev. Lett. 94 (2005) 131301 [astro-ph/ 0410359 ].

[106] A. Birkedal, K. T. Matchev, M. Perelstein and A. Spray, Robust gamma ray signature of WIMP dark matter, [hep-ph/0507194].

[107] L. Roszkowski, R. R. de Austri, J. Silk and R. Trotta, On prospects for dark matter indirect detection in the constrained MSSM, [arXiv:0707.0622].

[108] H. A. Mayer-Hasselwander et al. (EGRET Collaboration), High-energy gamma ray emission from the galactic center, Astron. \& Astrophys. 335 (1998) 161.

[109] See the web sites http://www-glast.slac.stanford.edu/software/IS/glast_lat_performance.htm http://www-glast.slac.stanford.edu/software/AnaGroup/burnett/performance.htm.

[110] See the web site of the AMS-02 collaboration http: //ams.cern.ch/.

[111] J. Nishimura et al., Emulsion chamber observations of primary cosmic ray electrons in the energy range $30 \mathrm{GeV}-1000 \mathrm{GeV}$, Astrophys. J. 238 (1980) 394.

[112] M. J. Ryan, J. F. Ormes and V. K. Balasubrahmanyan, Cosmic ray proton and helium spectra above 50 GeV, Phys. Rev. Lett. 28 (1972) 985.

[113] P. Chardonnet, P. Salati, J. Silk, I. Grenier and G. Smoot, The gamma ray galactic diffuse radiation and Cerenkov telescopes, Astrophys. J. 454 (1995) 774. 
[114] P. Sreekumar et al., EGRET observations of the extragalactic gamma ray emission, Astrophys. J. 494 (1998) 523

[115] F. Aharonian et al. (HESS Collaboration), Very high energy gamma rays from the direction of Sagittarius A*, Astron. \& Astrophys. 425 (2004) L13 [astro-ph/ 0408145 ].

[116] F. Aharonian et al. (HESS Collaboration), Discovery of very-high-energy gamma rays from the galactic centre ridge, Nature 439 (2006) 695 [astro-ph / 0603021 ].

[117] T. Bringmann and S. Hofmann, Thermal decoupling of WIMPs from first principles, JCAP 0407 (2007) 016 [hep-ph/0612238].

[118] V. Berezinsky, V. ÊDokuchaev and Y. Eroshenko, Small-scale clumps in the galactic halo and dark matter annihilation, Phys. Rev. D68 (2003) 103003 [astro-ph / 0301551 ].

[119] C. Boehm, P. Fayet and R. Schaeffer, Constraining dark matter candidates from structure formation, Phys. Lett. B518 (2001) 8 [astro-ph/ 0012504 ].

[120] S. Hofmann, D. J. Schwarz and H. Stoecker, Damping scales of neutralino cold dark matter, Phys. Rev. D64 (2001) 083507 [astro-ph / 0104173 ].

[121] S. Profumo, K. Sigurdson and M. Kamionkowski, What mass are the smallest protohalos ?, Phys. Rev. Lett. 97 (2006) 031301 [astro-ph/0603373].

[122] J. Diemand, B. Moore and J. Stadel, Earth-mass dark-matter haloes as the first structures in the early universe, Nature 433 (2005) 389 [astro-ph / 0501589 ].

[123] J. Diemand, M. Kuhlen and P. Madau, Early supersymmetric cold dark matter substructure, Astrophys. J. 649 (2006) 1 [astro-ph/0603250].

[124] J. Diemand, M. Kuhlen and P. Madau, Formation and evolution of galaxy dark matter halos and their substructure, Astrophys. J. 667 (2007) 859 [astro-ph/ 0703337 ].

[125] V. Berezinsky, V. ÊDokuchaev and Y. Eroshenko, Destruction of small-scale dark matter clumps in the hierarchical structures and galaxies, Phys. Rev. D73 (2006) 063504 [astro-ph/0511494].

[126] H. Zhao, D. Hooper, G. W. Angus, J. E. Taylor and J. Silk, Tidal disruption of the first dark microhalos, Astrophys. J. 654 (2007) 697 [astro-ph / 0508215 ].

[127] G. W. Angus and H. Zhao, Analysis of galactic tides and stars on CDM microhalos, Mon. Not. Roy. Astron. Soc. 375 (2007) 1146 [astro-ph / 0608580 ].

[128] T. Goerdt, O. Y. Gnedin, B. Moore, J. Diemand and J. Stadel, The survival and disruption of CDM micro-haloes : implications for direct and indirect detection experiments, Mon. Not. Roy. Astron. Soc. 375 (2007) 191 [astro-ph/0608495].

[129] K. Agashe and G. Servant, Warped unification, proton stability and dark matter, Phys. Rev. Lett. 93 (2004) 231805 [hep-ph/0403143].

[130] D. Hooper, J. E. Taylor and J. Silk, Can supersymmetry naturally explain the positron excess?, Phys. Rev. D69 (2004) 103509 [hep-ph/ 0312076 ].

[131] D. Hooper and G. D. Kribs, Kaluza-Klein dark matter and the positron excess, Phys. Rev. D70 (2004) 115004 [hep-ph/0406026].

[132] J. Diemand, M. Kuhlen and P. Madau, Dark matter substructure and gamma ray annihilation in the Milky Way halo, Astrophys. J. 657 (2007) 262 [astro-ph/ 0611370 ]. 
[133] V. Berezinsky, V. ÊDokuchaev and Y. Eroshenko, Anisotropy of dark matter annihilation with respect to the galactic plane, JCAP 0707 (2007) 011 [astro-ph/ 0612733 ].

[134] G. Bertone, A. R. Zentner and J. Silk, A new signature of dark matter annihilations : gamma rays from intermediate-mass black holes, Phys. Rev. D72 (2005) 103517 [astro-ph/ 0509565$].$

[135] G. Bertone, Prospects for detecting dark matter with neutrino telescopes in intermediate mass black holes scenarios, Phys. Rev. D73 (2006) 103519 [astro-ph / 0603148 ].

[136] P. Brun, G. Bertone, J. Lavalle, P. Salati and R. Taillet, Antiproton and positron signal enhancement in dark matter mini-spikes scenarios, Phys. Rev. D76 (2007) 083506 [arXiv : 0704 . 254 3].

[137] M. Coleman Miller and E. J. M. Colbert, Intermediate-mass black holes, Int. J. Mod. Phys. D13 (2004) 1 [astro-ph/0308402].

[138] P. Gondolo and J. Silk, Dark matter annihilation at the galactic center, Phys. Rev. Lett. 83 (1999) 1719 [astro-ph/9906391].

[139] P. Ullio, H. Zhao and M. Kamionkowski, A Dark-matter spike at the galactic center ?, Phys. Rev. D64 (2001) 043504 [astro-ph/ 0101481 ].

[140] D. Merritt, M. Milosavljevic, L. Verde and R. Jimenez, Dark matter spikes and annihilation radiation from the galactic center, Phys. Rev. Lett. 88 (2002) 191301 [astro-ph/ 0201376 ].

[141] D. Merritt, Evolution of the dark matter distribution at the galactic center, Phys. Rev. Lett. 92 (2004) 201304 [astro-ph/0311594].

[142] G. Bertone and D. Merritt, Time-dependent models for dark matter at the galactic center, Phys. Rev. D72 (2005) 103502 [astro-ph/0501555].

[143] J. Lavalle, Q. Yuan, D. Maurin and X. J. Bi, Full calculation of clumpiness boost factors for antimatter cosmic rays in the light of $\Lambda C D M N-$ body simulation results, Astron. \& Astrophys. 479 (2008) 427 [arXiv: 0709 .3634]. 